\title{
WestVirginiaUniversity
}

THE RESEARCH REPOSITORY @ WVU

Graduate Theses, Dissertations, and Problem Reports

2010

\section{High rank tensor and spherical harmonic models for diffusion MRI processing}

Inas A. Yassine

West Virginia University

Follow this and additional works at: https://researchrepository.wvu.edu/etd

\section{Recommended Citation}

Yassine, Inas A., "High rank tensor and spherical harmonic models for diffusion MRI processing" (2010). Graduate Theses, Dissertations, and Problem Reports. 3205.

https://researchrepository.wvu.edu/etd/3205

This Dissertation is protected by copyright and/or related rights. It has been brought to you by the The Research Repository @ WVU with permission from the rights-holder(s). You are free to use this Dissertation in any way that is permitted by the copyright and related rights legislation that applies to your use. For other uses you must obtain permission from the rights-holder(s) directly, unless additional rights are indicated by a Creative Commons license in the record and/ or on the work itself. This Dissertation has been accepted for inclusion in WVU Graduate Theses, Dissertations, and Problem Reports collection by an authorized administrator of The Research Repository @ WVU.

For more information, please contact researchrepository@mail.wvu.edu. 


\title{
HIGH RANK TENSOR AND SPHERICAL HARMONIC MODELS FOR DIFFUSION MRI PROCESSING
}

\author{
Inas A. Yassine \\ Dissertation submitted to the \\ College of Engineering and Mineral Resources \\ at West Virginia University \\ in partial fulfillment of the requirements \\ for the degree of
}

Doctor of Philosophy in Electrical Engineering

\author{
McGraw, Timothy , Ph.D., Chair \\ Ross, Arun, Ph.D. \\ Schmid, Natalia, Ph.D. \\ Mukdadi, Sam , Ph.D. \\ Adjeroh, Donald, Ph.D.
}

Lane Department of Computer Science and Electrical Engineering Morgantown, West Virginia University 2010 


\begin{abstract}
HIGH RANK TENSOR AND SPHERICAL HARMONIC MODELS

FOR DIFFUSION MRI PROCESSING
\end{abstract}

Inas A. Yassine

Diffusion tensor imaging (DTI) is a non-invasive quantitative method of characterizing tissue micro-structure. Diffusion imaging attempts to characterize the manner by which the water molecules within a particular location move within a given amount of time. Measurement of the diffusion tensor (D) within a voxel allows a macroscopic voxel-averaged description of fiber structure, orientation and fully quantitative evaluation of the microstructural features of healthy and diseased tissue.

The rank two tensor model is incapable of resolving multiple fiber orientations within an individual voxel. This shortcoming of single tensor model stems from the fact that the tensor possesses only a single orientational maximum. Several authors reported this non-mono-exponential behavior for the diffusion-induced attenuation in brain tissue in water and N-Acetyl Aspartate (NAA) signals, that is why the Multi-Tensor, Higher Rank Tensor and Orientation Distribution Function (ODF) were introduced.

Using the higher rank tensor, we will propose a scheme for tensor field interpolation which is inspired by subdivision surfaces in computer graphics. The method applies to Cartesian tensors of all ranks and imposes smoothness 
on the interpolated field by constraining the divergence and curl of the tensor field. Results demonstrate that the subdivision scheme can better preserve anisotropicity and interpolate rotations than some other interpolation methods. As one of the most important applications of DTI, fiber tractography was implemented to study the shape geometry changes. Based on the divergence and curl measurement, we will introduce new scalar measures that are sensitive to behaviors such as fiber bending and fanning.

Based on the ODF analysis, a new anisotropy measure that has the ability to describe multi-fiber heterogeneity while remaining rotationally invariant, will be introduced, which is a problem with many other anisotropy measures defined using the ODF. The performance of this novel measure is demonstrated for data with varying Signal to Noise Ratio (SNR), and different material characteristics. 


\section{ACKNOWLEDGMENTS}

The writing of a dissertation can be a lonely and isolating experience, yet it is obviously not possible without the personal and practical support of numerous people. Thus my sincere gratitude goes to my parents, all my family members, all my friends for their love and support over the last few years.

Many people on the faculty and staff of the LCSEE department assisted and encouraged me in various ways during my course of studies. I am especially grateful to Profs. Natalia Schmid, and Arun Ross for all that they have taught me. I was also greatly inspired pedagogically by Prof. Mark Jerabek, for whom I was a Teaching Assistant for two years, and I thank the students whom I was privileged to teach and from whom I also learned much.

My graduate studies would not have been the same without the social and academic challenges and diversions provided by all my student-colleagues in the Lane Department of Computer Science and Electrical Engineering. I am particularly thankful to all my lab mates. We studied, relaxed, and traveled as well together.

I would like also o share my deepest gratitude to dr. Yasser Kadah who supported me morally and kept asking about me, even if I am lazy enough for not replying.

I am heartily thankful to my supervisor, Dr. Tim Mcgraw, whose encouragement, supervision and support from the preliminary to the concluding level enabled me to develop an understanding of the subject. 
I would like also to thank my family: my parents, Ahmed Yassine, Nashwa Negm, for giving me life in the first place, for educating me with aspects from both arts and sciences, for unconditional support and encouragement to pursue my interests, even when the interests went beyond boundaries of language, sports and arts and My Uncle Mohab Negm, who was regularly and frequently flying from Egypt just to make sure that am fine.

Last, but not least, all my friends in Morgantown, for their support, sharing their experience with me, for listening to my complaints and frustrations, and for believing in me. 


\section{Contents}

Table of Contents vi vi

List of Figures $\quad$ ix

1 Thesis Objective and Contributions 1

1.1 Thesis Objective . . . . . . . . . . . . . . . . . . . . 1

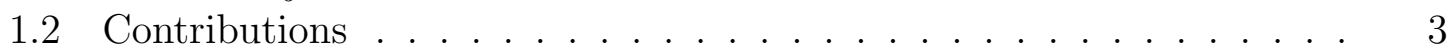

2 Magnetic Resonance Imaging and Diffusion Tensor MRI 4

2.1 Magnetic Resonance Imaging . . . . . . . . . . . . . . . . . . 4

2.1.1 Properties of Atomic Nuclei . . . . . . . . . . . . . . 5

2.1.2 Net Magnetization Vector ............... 5

2.1.3 Radiofrequency Field . . . . . . . . . . . . . . . 6

2.1.4 $T_{1}$ Relaxation ................... 8

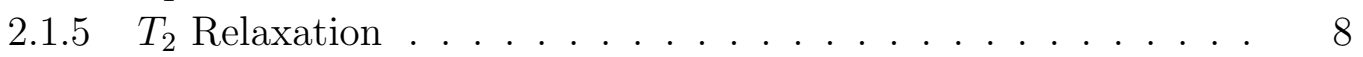

2.1.6 Rotating Frame of Reference . . . . . . . . . . . . . . . . . 9

2.1.7 Spatial Characteristics of Magnetic Resonance Images . . . . . 9

2.1.8 Spatial Localization using Magnetic Field Gradient . . . . . . 10

2.1.8.1 Slice Selection . . . . . . . . . . . . . . 10

2.1.8.2 Frequency Encoding . . . . . . . . . . . . . 12

2.1.8.3 Phase Encoding . . . . . . . . . . . . . . . 13

2.1.9 Two Dimensional Fourier transform Magnetic Resonance . . . 13

2.1.10 Sequence Timing . . . . . . . . . . . . . . . . . . . . . 14

2.1.10.1 Repetition Time . . . . . . . . . . . . . . . 15

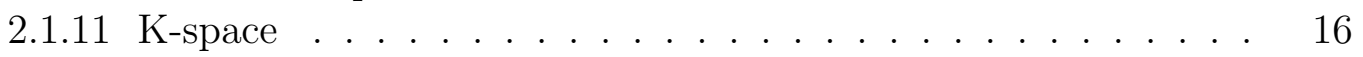

2.1 .12 Image Reconstruction . . . . . . . . . . . . . . . . . . . . 17

2.1.13 Image Contrast . . . . . . . . . . . . . . . . . . . . . . . . . . . . . . . . . 18

2.1.14 Pulse Sequence . . . . . . . . . . . . . . . . . . . . . . . . . . . . . . . . . . . 19

2.1 .15 Image Noise . . . . . . . . . . . . . . . . . . . . . . . . . . . 19

2.1.15.1 Statistical (or Random) Noise . . . . . . . . . . 20

2.1.15.2 Systematic Noise . . . . . . . . . . . . . . 20

2.1.16 Safety and Bioeffects . . . . . . . . . . . . . . . . . . 20

2.1.17 MRI Modalities . . . . . . . . . . . . . . . . . . 21 
2.2 Diffusion Weighted and Diffusion Tensor MRI . . . . . . . . . . . 21

2.2.1 Diffusion Weighted Imaging (DWI) . . . . . . . . . . . . . . 23

2.2.1.1 Anisotropy in DWI . . . . . . . . . . . . . 27

2.2.1.2 Applications of DWI . . . . . . . . . . . . 29

2.2.2 Diffusion Tensor Imaging . . . . . . . . . . . . . . . . . . 29

2.2.2.1 Pulsed Gradient Echo Pulse Sequence . . . . . . . . 30

2.2.2.2 Diffusion Tensor Reconstruction . . . . . . . . . . . . 34

2.2.2.3 Diffusion Tensor Eigen System . . . . . . . . . . . 34

2.2.2.4 Diffusion in an Isotropic Medium . . . . . . . . . 35

2.2.2.5 Diffusion in an Anisotropic Medium . . . . . . . . 35

2.2.2.6 Quantitative Parameters Obtained by DT MRI . . . 36

2.2.2.7 Diffusion Profiles of Various Brain Tissues . . . . . . 38

3 Multi- and Higher Rank Tensors $\quad 40$

3.1 Introduction . . . . . . . . . . . . . . . . . . 40

3.2 Multi Tensors . . . . . . . . . . . . . . . . . . . 41

3.3 Higher Order Tensors . . . . . . . . . . . . . . . . . . . . 43

3.3.1 Generalized Anisotropy . . . . . . . . . . . . . . . 44

4 Tensor Field Subdivision 48

4.1 Introduction . . . . . . . . . . . . . . . . . . . 48

4.1.1 Log Euclidian Interpolation . . . . . . . . . . . . . . . 49

4.1.2 Geodesic Interpolation . . . . . . . . . . . . . . . . . 50

4.1 .3 Tensor Spline Interpolation . . . . . . . . . . . . . . . . . . 51

4.1.4 Geodesic-Loxodrome Subdivision . . . . . . . . . . . . 53

4.2 Divergence and Curl Minimizing Subdivision . . . . . . . . . . . 54

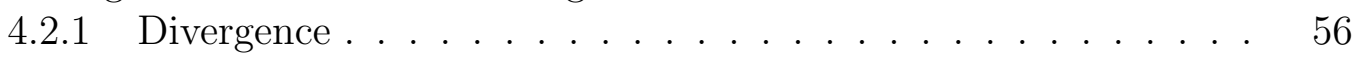

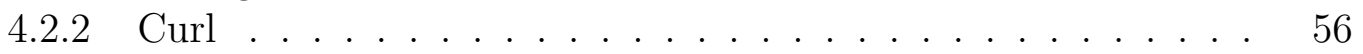

4.2 .3 Vector Field Subdivision . . . . . . . . . . . . . . . 57

4.2.4 Tensor Field Subdivision . . . . . . . . . . . . . . . . . 61

4.2.4.1 Rank-2 Tensor Subdivision . . . . . . . . . . . 63

4.2.4.2 Rank-4 Tensor Subdivision . . . . . . . . . . . 65

4.2.4.3 Bézier Curves and Splines ........... . . 65

4.2.4.4 Minimization ................ 67

4.2.5 Subdivision Results and Discussion . . . . . . . . . . . . . . 69

4.3 Fiber Tracking and Shape Geometry . . . . . . . . . . . . . . . . . . 79

4.3.1 Fiber Assignment by Continuous Tracking Technique (FACT) 81

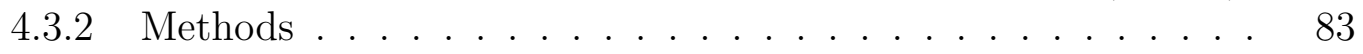

4.3.3 Results and Discussion . . . . . . . . . . . . . . . 84

5 New Measures Based on Divergence and Curl 86

5.1 Introduction . . . . . . . . . . . . . . . . . 86

5.2 Methods . . . . . . . . . . . . . . . . . . . . . . 88 
5.3 Results . . . . . . . . . . . . . . . . . . . . . 91

6 Orientation Distribution Function and Anisotropy Measure 99

6.1 Introduction . . . . . . . . . . . . . . . . . 101

6.2 Literature Review . . . . . . . . . . . . . . . . . . . . . . 102

6.2.1 Diffusion Spectrum Imaging (DSI) . . . . . . . . . . . . . . . . 102

6.2.2 Persistent Angular Structure(PAS) . . . . . . . . . . . . . . 103

6.2.3 Diffusion Orientation Transform (DOT) . . . . . . . . . . . . 104

6.2.4 Tensor Distribution Function . . . . . . . . . . . . . . 105

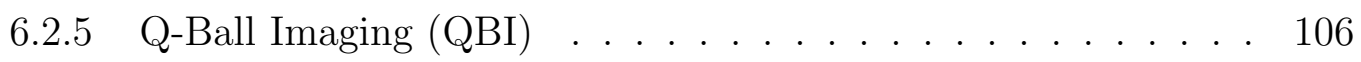

6.3 Anisotropy Measurements . . . . . . . . . . . . . . . 108

6.4 Methods . . . . . . . . . . . . . . . . . . 110

6.4.1 Spherical Harmonics . . . . . . . . . . . . . . . . . 110

6.4.1.1 Legendre Function . . . . . . . . . . . . . . . . 111

6.4.1.2 Spherical Harmonics $(\mathrm{SH})$. . . . . . . . . . . 112

6.4.2 ODF Calculation . . . . . . . . . . . . . . . 114

6.4.3 Orientation Distribution Function Anisotropy Measure [ODFA] 116

6.4.4 Monte Carlo Simulation . . . . . . . . . . . . . . . 117

6.5 Results and Discussion . . . . . . . . . . . . . . . . . 118

7 Conclusion and Future Work $\quad 126$

7.1 Conclusions . . . . . . . . . . . . . . . . . 126

7.2 Future Work . . . . . . . . . . . . . . . . . 128

$\begin{array}{lr}\text { Bibliography } & 129\end{array}$

$\begin{array}{ll}\text { Index } & 145\end{array}$ 


\section{List of Figures}

2.1 Nuclear magnetism. Nuclei with net spin (I) have a characteristic magnetic moment $(\mathrm{m})$ and an associated magnetic field, similar to a dipole, such as a bar magnet. . . . . . . . . . . . . . . . .

2.2 Nuclear magnetism. Nuclei with net spin (I) have a characteristic magnetic moment $(\mathrm{m})$ and an associated magnetic field, similar to a dipole, such as a bar magnet. . . . . . . . . . . . . . 6

2.3 Tipping of longitudinal magnetization (a)into transverse magnetization by a magnetic field, B1, associated with an RF wave or pulse (b). . . 7

2.4 Transverse magnetization and MR signals. . . . . . . . . . . . . 8

2.5 the Relaxation of $M_{z}$ and $M_{x y}$ with time in the longitudinal and transverse direction respectively. . . . . . . . . . . . . . . . 9 9

2.6 Magnetic field gradients in the $\mathrm{x}, \mathrm{y}$, and $\mathrm{z}$ directions within a cylindrical magnet. . . . . . . . . . . . . . . . . . 10

2.7 Effect of gradient amplitude and bandwidth on slice thickness. . . . . 11

2.8 Common shape for envelope of slice-selective pulses in MRI which excite an approximately rectangular distribution of spins. . . . . . . . . 12

2.9 Pulse timing diagram for spin-echo pulse sequence in conventional Fourier. . . . . . . . . . . . . . . . . 15

2.10 Representing difference between sampling time and sampling frequency. 16

2.11 Influence of k-space regions on spatial properties. . . . . . . . . . . . 17

2.12 Difference between repetition time and Sampling time. . . . . . . . . 17

2.13 Reconstruction of $2 \mathrm{D} \mathrm{k}$ space into 2D MR image. . . . . . . . . . . 18

2.14 Timing diagram for ST SE DW pulse sequences and series of DW images of the human brain. . . . . . . . . . . . . . . . 31

2.15 Principles of Navigation in DWIs. . . . . . . . . . . . . . . 32

2.16 Diffusion-encoding directions generated by the pulse sequence are spherical tessellations of an icosahedron. . . . . . . . . . . . . . . 33

2.17 Homogenous isotropic tissue diffusion profile. . . . . . . . . . . . . 38

2.18 Homogenous isotropic tissue diffusion profile. . . . . . . . . . . 39

2.19 Anisotropic white matter diffusion profile. . . . . . . . . . . . . . 39 
4.1 Interpolation between 2D tensors of eigenvalues $(5,1)$ horizontally and $(1,50)$ at 45 degrees. Left: Interpolation in the standard matrix space (interpolation of the coefficients). Right: Geodesic interpolation in Riemannian space . . . . . . . . . . . . . . . . . .

4.2 First three steps of CatmullClark subdivision of a cube with subdivision

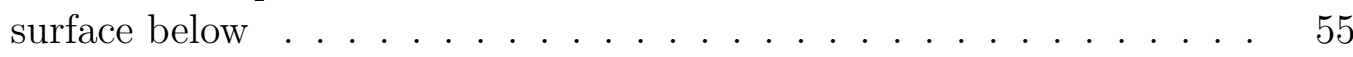

4.3 Diagram illustrating divergence . . . . . . . . . . . . . . . . . . 56

4.4 Diagram illustrating vorticity in a fluid . . . . . . . . . . . . 57

4.5 Illustration of the subdivision process in 2D. The first subdivision iteration replaces the $2 \times 2$ grid of vectors $\left(v^{0}\right)$ with a $3 \times 3$ grid of vectors $\left(v^{1}\right)$. The vectors in the corners of the domain (white background) are interpolated. The remaining 5 vectors are computed by minimizing the divergence and curl of the field. The next subdivision step would interpolate all 9 vectors. The process can be repeated to obtain $v^{n}$, a grid of size $2^{n}+1 \times 2^{n}+1 \ldots \ldots \ldots$. . . . . . . . . . . . . . . . . . . . 59

4.6 Sparse A and B matrices. . . . . . . . . . . . . . . . . . 60

4.7 Vector Field Subdivision of rotational vector. The field to be interpolated (top left) is subdivided 3 times (results shown top to down and left to right).

4.8 Vector Field Subdivision of nonsolenoidal vector. The field to be interpolated (top left) is subdivided 3 times (results shown top to down and left to right).

4.9 Vector Field Subdivision of rotational vector. The field to be interpolated (top left) is subdivided 3 times (results shown top to down and left to right).

4.10 Vector Field Subdivision of nonsolenoidal vector. The field to be interpolated (top left) is subdivided 3 times (results shown top to down and left to right). . . . . . . . . . . . . . 63

4.11 Input and Output voxels in case of 2D subdivision . . . . . . . . . 68

4.12 Input and Output voxels in case of 3D subdivision . . . . . . . . . . . 68

4.13 Rank 2 tensor field interpolation. Linear interpolation (left), LogEuclidean interpolation (center), 2 subdivision steps (right). . . . . .

4.14 Rank 2 tensor field interpolation. Linear interpolation (left), LogEuclidean interpolation (center), 2 subdivision steps (right). . . . . .

4.15 Solenoidal, Rank 2 tensor, Top: (Right) Ground truth slice, (Left) Input slice, Bottom: (Right) Subdivision Output, (Left)Linear Interpolation Output. . . . . . . . . . . . . . . . .

4.16 Exponential decay, Rank 2 tensor, Top: (Right) Ground truth slice, (Left) Input slice, Bottom: (Right) Subdivision Output, (Left)Linear Interpolation Output. . . . . . . . . . . . . . .

4.17 kissing fibers, Rank 2 tensor, Top: (Right) Ground truth slice, (Left) Input slice, Bottom: (Right) Subdivision Output, (Left)Linear Interpolation Output. 
4.18 Bézier spline Interpolation, Rank 2 tensor results with Degree $\mathrm{n}=1$ (left) and $n=2$ (right). . . . . . . . . . . . . . . . .

4.19 Bézier spline Interpolation, Rank 2 tensor results with Degree $n=3$ (left) and $n=4$ (right). . . . . . . . . . . . . . . . . . . 74

4.20 Bézier spline Interpolation, Rank 2 tensor results with Degree $\mathrm{n}=5$ (left) and $n=6$ (right). . . . . . . . . . . . . . . . . .

4.21 Bézier spline Interpolation, Rank 2 tensor results with Degree $n=7$ (left) and Order 2 tensor subdivision results (right). . . . . . . . . . . 75

4.22 Rank-4 tensor interpolation, Degree $\mathrm{n}=7$ (left) and subdivision (right). The background image is generalized anisotropy. . . . . . . . . . . 76

4.23 Interpolation of real data taken from sample within the blue box (top). Detail of the sample (bottom left), interpolated field (bottom right) .

4.24 Top: A brain dissection showing the structure of white matter (from The Virtual Hospital, University of Iowa). Bottom: A coronal T2 slice combined with a plot of the eigenvector corresponding to the largest eigenvalue. . . . . . . . . . . . . . . . . .

4.25 Tracking multiple fiber paths, following the direction of maximum diffusion. Visualized using the 3-D Viewer. . . . . . . . . . . . . . . . . 80

4.26 FACT Tracking Example . . . . . . . . . . . . . . . . . . . 83

4.27 Circular Tract for the downsampled, Linearly Interpolated and the Subdivided volumes . . . . . . . . . . . . . . . . .

4.28 Spiral Tract for the downsampled, Linearly Interpolated and the Subdivided volumes . . . . . . . . . . . . . . 85

5.1 Vortices and sources used to construct the synthetic field. . . . . . . . 91

5.2 Helmholtz decomposition results for rank-2 synthetic tensor field.[top: right: Tensor, Left: Curl, Bottom: right:, left: Harmonic] . . . . . . .

5.3 Helmholtz decomposition results for rank-4 synthetic tensor field.[top: right: Tensor, Left: Curl, Bottom: right:, left: Harmonic] . . . . . . . 94

5.4 Axial slice of real data, rank 2. [left:Curl $\psi$, Right: Grad $\phi$ ]. . . . . . 94

5.5 A comparison of FA of white-matter structure for an axial slice. ILF: interior longitudinal fasciculus, SFO: superior fronto-occipital fasciculus, SCC: splenium of corpus callosum, RCB/LCB: right/left cingulum bundle, ATR: Anterior thalamic radiation. . . . . . . . . . . .

5.6 A comparison of $\| C$ url $D_{\psi} \|$ of white-matter structure for an axial slice. ILF: interior longitudinal fasciculus, SFO: superior fronto-occipital fasciculus, SCC: splenium of corpus callosum, RCB/LCB: right/left cingulum bundle, ATR: Anterior thalamic radiation. . . . . . . . . .

5.7 A comparison of $\left\|D i v D_{\psi}\right\|$ of white-matter structure for an axial slice. ILF: interior longitudinal fasciculus, SFO: superior fronto-occipital fasciculus, SCC: splenium of corpus callosum, RCB/LCB: right/left cingulum bundle, ATR: Anterior thalamic radiation. . . . . . . . . . 
5.8 Scalar measures of the rank-4 tensor field for an axial slice of real huma brain.[left: $\left\|D_{\psi}\right\|$, Right: $\left.\left\|D_{\psi}\right\|\right]$. . . . . . . . . . . . . . . . 98

6.1 First Four bands $l=0, \ldots, 3$ of the associated Legendre Polynomials . . 112

6.2 GA and ODFA values corresponding to FA . . . . . . . . . . . . . 119

6.3 Rotation variance of the closed form of the ODFA for different Anisotropy values . . . . . . . . . . . . . . . . . . . . . . . . . 120

6.4 Rotation variance of the closed form of the ODFA for different Anisotropy values . . . . . . . . . . . . . . . . . . . . . . . . 121

6.5 Effect of noise on the FA and ODFA for White Matter tissue . . . . 123

6.6 Effect of noise on the FA and ODFA for Gray Matter tissue . . . . . 123

6.7 Effect of noise on the FA and ODFA for Cerebrospinal Fluid tissue . 124

6.8 Anisotropy measurements for an axial human brain slice: Top [FA

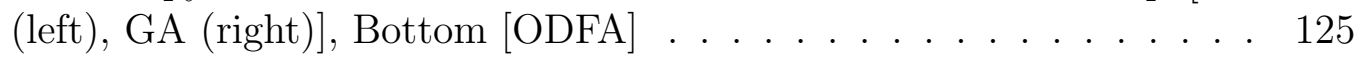




\section{Chapter 1}

\section{Thesis Objective and Contributions}

\subsection{Thesis Objective}

The single tensor model is incapable of resolving multiple fiber orientations within an individual voxel. This shortcoming of the tensor model stems from the fact that the tensor possesses only a single orientational maximum, i.e., the major eigenvalue of the diffusion tensor. At the millimeter-scale resolution typical of DTI, the volume of cerebral white matter containing such intravoxel orientational heterogeneity (IVOH) may be considerable given the widespread divergence and convergence of fascicles. The abundance of IVOH at the millimeter scale can be further appreciated by considering the ubiquity of oblate (pancake-shaped) diffusion tensors in DTI, a hypothesized indicator of IVOH. Several authors reported a non-mono-exponential behavior for the diffusion-induced attenuation in brain tissue in water and $\mathrm{N}$-acetyl aspartate NAA signals. Their study was based on a 1-D diffusion experiment and the results were fitted to bi- or tri-exponential functions. They indicated that high $\mathrm{b}$ 
values reveal more complex behavior and therefore their fit depended on the value of $\mathrm{b}$ used in the measurement. This gives us the motivation to use the high order tensor and the orientation distribution in our analysis.

In order to avoid non-unique variations in measuring diffusivity parameters with the positioning of the subject, a more general characterization of the diffusion process was introduced based on diffusion tensors.

Since voxels in clinical DT images are often quite anisotropic and the voxel size is in the $m m$ scale although the brain fibers width are in the $n m$ scale, a preliminary resampling step with an adequate interpolation method or subdivision is therefore important for such algorithms. Proper interpolation methods are also required to generalize to the tensor case usual registration techniques used on scalar or vector images.

We want to derive the methodology and apparatus for subdivision of higher rank multi-axon fiber tracking based on high angular resolution diffusion-weighted acquisitions. Given the characteristics of axonal membranes, the diffusion of water inside each axon is preferred along the direction of the axon rather than across it, divergence and curl properties of the fibers, a least square subdivision algorithm preserving anisotropicity of the compartments, is used.

Further analysis of the orientation distribution function is also important as a step for fiber tractography, which is the most known application of DTI imaging. Through this analysis, a novel anisotropic measurement is derived based on the ODF that solves the problem of the rotationally invariance that was the main problem of all other anisotropy measurement based on the ODF. This measure preserves the difference in anisotropy due to multi compartment behavior in voxels. Experimental analysis were used to check the rotational invariance and effect of noisy signal on the new measure estimation. 


\subsection{Contributions}

A new Subdivision Algorithm has been developed, that can be extended to higher rank tensor. Solution must be optimal in least-square sense and efficient in terms of computational requirements. the new algorithm was compared to the the linearly interpolated and the log Euclidian interpolated volumes, Anisotropy smoothness, Tensor Frobenuis norm, and Tract shape geometry were calculated.

A new structural measurement, that was based on the divergence and curl, was proposed. this measure has the advantage of being extended to tensor of any rank.

Finally, A new anisotropic measure that is based on the SH-ODF was proposed. It has the advantage of being rotationally invariant and not being based on model estimation. A Closed form for the new measurement was calculated using Mathematica. the behavior of this measure under different conditions of SNR for different standard brain tissue was estimated. 


\section{Chapter 2}

\section{Magnetic Resonance Imaging and Diffusion Tensor MRI}

\subsection{Magnetic Resonance Imaging}

Magnetic resonance (MR) is a phenomenon involving magnetic fields and radiofrequency (RF) electromagnetic waves. It was discovered in 1946 independently by Bloch and co-workers at Stanford and by Purcell at Harvard. Since then magnetic resonance imaging (MRI) has been a useful tool, especially for analytical chemistry and biochemistry, thanks to the discovery of the chemical shift. MRI can produce images with excellent contrast between soft tissues and high spatial resolution in every direction. MRI uses electromagnetic radiation to probe inside the human body. Furthermore, the radiation has low energy and appears to be safe under normal operating conditions [1].

This chapter is a quick review on the fundamentals of MRI. 


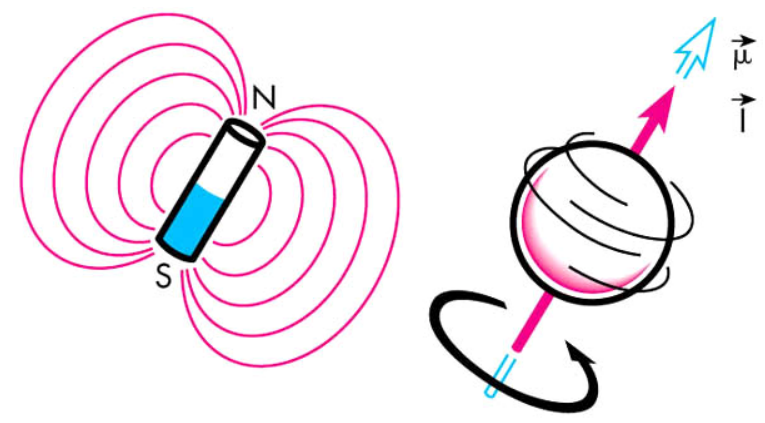

Figure 2.1 Nuclear magnetism. Nuclei with net spin (I) have a characteristic magnetic moment $(\mathrm{m})$ and an associated magnetic field, similar to a dipole, such as a bar magnet.

\subsubsection{Properties of Atomic Nuclei}

At the core of atoms and accommodating most of the elemental mass is the nucleus, consisting of neutrons and protons. Nuclei with an odd number of neutrons or protons possess spin-angular momentum, have a magnetic moment, m, characterizing the magnetic field surrounding the nucleus. The magnetic field attributed to a nucleus is analogous to that from a bar magnet as shown in figure (2.1) [1].

\subsubsection{Net Magnetization Vector}

When exposed to a static magnetic field $B_{0}$, the randomly oriented magnetic dipoles tend to align with the magnetic field. The phases of an ensemble of magnetic moments are random, as shown in Figure (2.2). Therefore the individual magnetic moments make up the surface of a double cone, and their joint alignment creates the net magnetization, M. The net magnetization is the vector sum of the individual magnetic moments, as follows:

$$
M=\sum_{i=1}^{N} P_{i} \mu_{i}
$$

where $\mu_{i}$ is the magnetic moment of the $i^{t h}$ state and $P_{i}$ is its population, which follows Boltzman statistics. 


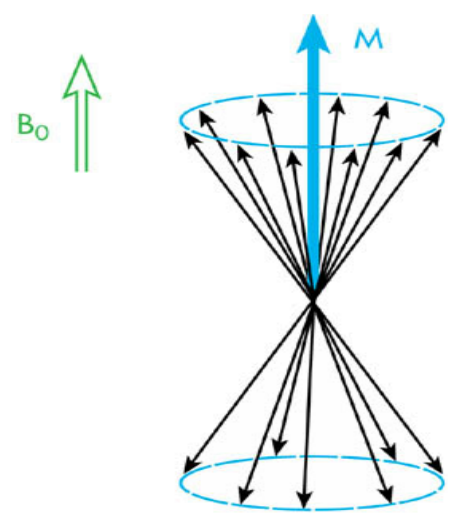

Figure 2.2 Nuclear magnetism. Nuclei with net spin (I) have a characteristic magnetic moment $(\mathrm{m})$ and an associated magnetic field, similar to a dipole, such as a bar magnet.

The net magnetization experiences a torque from the magnetic field analogous to a spinning top in the earth's gravitational field. As a result, the magnetization precesses around the axis of the magnetic field at a special frequency called the Larmor frequency $[1,2]$.

$$
\omega_{0}=\gamma B_{0}
$$

where $\gamma$ is the gyromagnetic ratio characteristic of the nuclear isotope [2].

\subsubsection{Radiofrequency Field}

MR can be detected only if transverse magnetization (magnetization perpendicular to $\left.B_{0}\right)$ is created because this transverse magnetization is time dependent and thus, according to Faraday's law of induction; can induce a voltage in a receiver coil. The longitudinal magnetization in thermal equilibrium is static and therefore does not meet the criteria for magnetic induction. Transverse magnetization is created when the Radio Frequency $[\mathrm{RF}]$ field of amplitude of $B_{1}$, rotating synchronously with the precessing spins, is applied as shown in Figure (2.3). If the B1 field rotates the net 


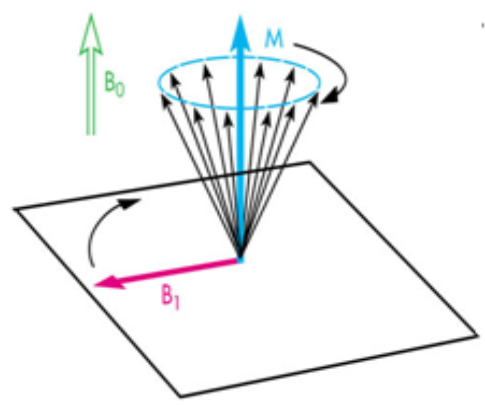

(a)

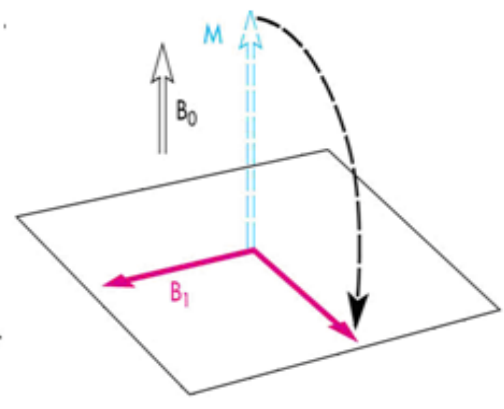

(b)

Figure 2.3 Tipping of longitudinal magnetization (a)into transverse magnetization by a magnetic field, B1, associated with an RF wave or pulse (b).

magnetization by 90 degrees, all of the longitudinal magnetization is converted to transverse magnetization $[1,3]$. Once the RF pulse is removed, the magnetization precesses about the static magnetic field at the Larmor frequency. The precessing magnetization can be detected as a time-varying electrical voltage across the ends of a coil of wire oriented as shown in Figure (2.4). The magnetization also decays exponentially with time-constant $T_{2}$. A simple model for this induced voltage can be calculated using equation shown here:

$$
V=k M_{0} \exp ^{2 i \pi f_{i} T} \exp ^{-t / T_{2}}
$$

where $\mathrm{k}$ is a constant and $\mathrm{i}=(-1)^{1 / 2}[1,2]$. The precessing transverse magnetization is represented by a complex number, which is composed of two numbers-the real part and the imaginary part. No special significance is attached to the terms real and imaginary. The induced voltage has the characteristics of a damped cosine and hence is also called free-induction decay (FID). The imaginary component of the transverse magnetization has the same phase as the B1 RF field and the real component is 90 degrees out of phase, as shown in Figure (2.5). [1] 


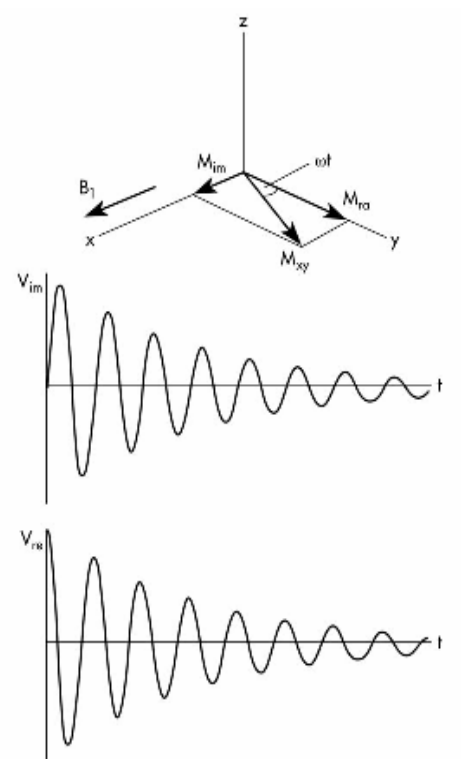

Figure 2.4 Transverse magnetization and MR signals.

\subsection{4 $T_{1}$ Relaxation}

RF stimulation causes nuclei to absorb energy, lifting them to the excited state. The nuclei can return to the ground state only by dissipating their excess energy to their surroundings, which is called the lattice. The process, which is aptly named spinlattice relaxation, describes the recovery of the longitudinal magnetization toward its equilibrium value $[1,4]$.

\subsection{5 $T_{2}$ Relaxation}

The transverse magnetization decays because its component magnetic moments get out of phase as a result of their mutual interaction. Anything that changes the magnetic field strength also changes the precessional frequency and causes a loss of phase coherence (dephasing) and shrinking of the transverse magnetization. A process called $T_{2}$ relaxation denotes the loss of phase coherence caused by interactions between neighboring magnetic moments [4]. Unlike $T_{1}$ relaxation, no energy is transferred from nuclei to the lattice in $T_{2}$ relaxation. Nuclei in the excited and ground state 


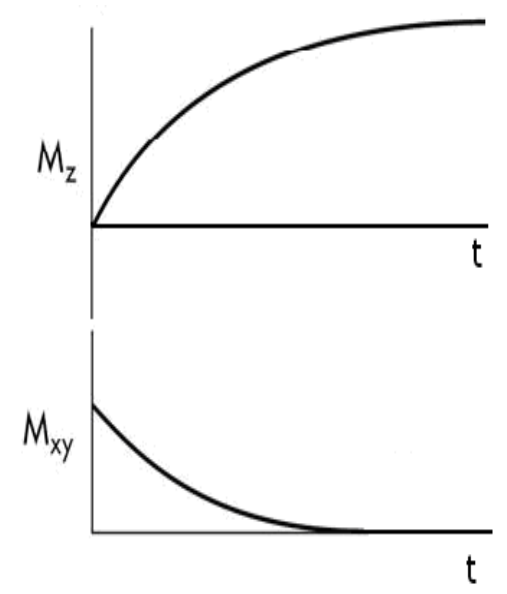

Figure 2.5 the Relaxation of $M_{z}$ and $M_{x y}$ with time in the longitudinal and transverse direction respectively.

may exchange energy with each other. The magnetic field strength influences $T_{2}$ much less than $T_{1}$, at least under the conditions encountered in MRI. Typically, $T_{2}$ in biological tissue ranges from approximately 50 to $100 \mathrm{~ms}$ [1].

\subsubsection{Rotating Frame of Reference}

The motion of the net magnetization vector during the action of the $B_{1}$ field is shown in a coordinate system in which the $\mathrm{x}$ and $\mathrm{y}$ axes rotate synchronously with the $B_{1}$ field. Such a rotating frame of reference greatly simplifies the description of the motion of the magnetization vector. In a static frame of reference, the tip of the magnetization vector spirals from the $\mathrm{z}$ axis onto the $\mathrm{xy}$ plane $[1,3]$.

\subsubsection{Spatial Characteristics of Magnetic Resonance Images}

Almost every MR image arises from Fourier imaging, which is an efficient and versatile technique for identifying the location of MR signals emanating from various regions of the body. It can create $2 \mathrm{D}$ and $3 \mathrm{D}$ MR images with various sizes and spatial characteristics. The images are calculated from digitized MR signals, which 


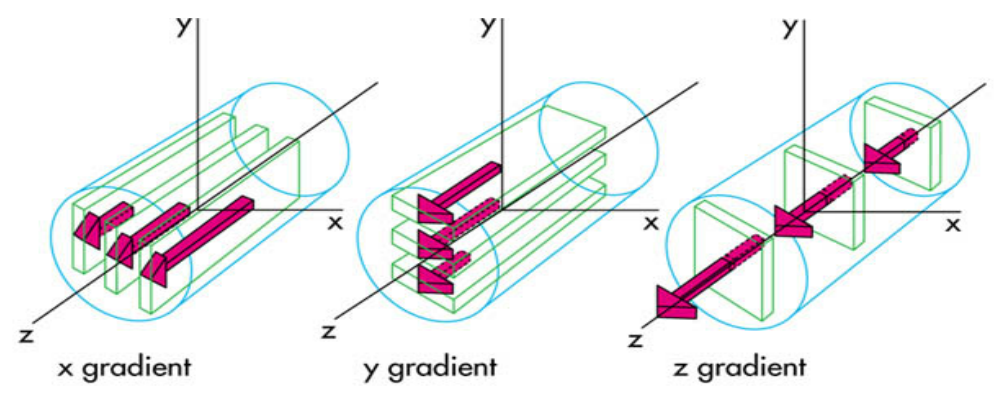

Figure 2.6 Magnetic field gradients in the $\mathrm{x}, \mathrm{y}$, and $\mathrm{z}$ directions within a cylindrical magnet.

are usually echoes. The next section describes how spatial information is encoded into these MR signals and then decoded during the calculation of an MR image in the image reconstruction process. Most MR images are presented as 2D planes partitioned into a grid of picture elements (voxels). The intensity of a pixel represents the strength of the MR signals emanating from the corresponding region [1].

\subsubsection{Spatial Localization using Magnetic Field Gradient}

Magnetic field gradients are activated briefly as pulses at carefully timed moments during MRI. A magnetic field gradient is a magnetic field that increases in strength along a particular direction. There are $\mathrm{x}, \mathrm{y}$, and $\mathrm{z}$ gradients, according to the direction along which the magnetic field changes strength as shown in Figure (2.6). The strength of a gradient refers to the rate at which its magnetic field changes with distance. Regardless of the direction of a gradient, its magnetic field is always directed along the $\mathrm{z}$ axis [1].

\subsubsection{Slice Selection}

Slice selection combines a magnetic field gradient and a specially shaped RF pulse to restrict MR signals to a slice instead of the entire region influenced by the transmitter coil. The gradient spreads out the Larmor frequency so that the frequencies contained 


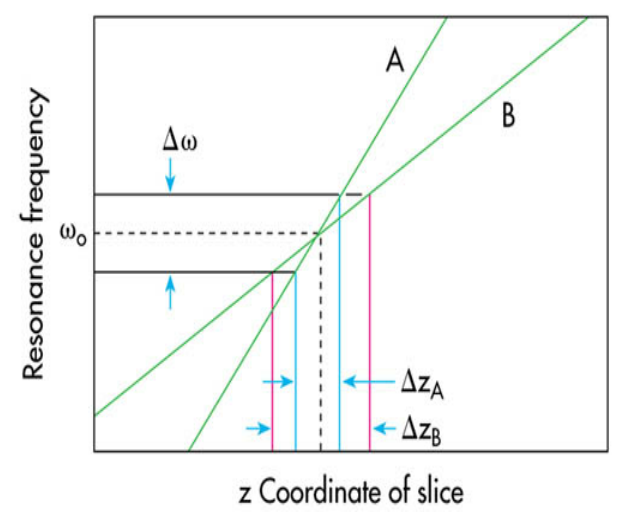

Figure 2.7 Effect of gradient amplitude and bandwidth on slice thickness.

in the RF pulse affect only a slice. Certain characteristics of the RF pulse and gradient affect the orientation, position, thickness, and actual shape of the slice [1].

The orientation of a slice depends on which of the three magnetic field gradients is activated during the RF pulse. If a patient is positioned head first and supine in a magnet, such as in Figure (2.6), an RF pulse in the presence of the $\mathrm{z}$ gradient creates a transverse slice. Slices are located where the Larmor frequency matches the frequency of the gradient pulse. The slice-selection gradient lowers the Larmor frequency on one side of the center of the magnet and raises it on the other side. Slice position is controlled by changing the frequency of the gradient pulse because changing the amplitude of the slice-selection gradient would inadvertently alter the thickness of the slice. Gradient pulses perturb magnetization within a band of Larmor frequencies matching the frequencies contained within the RF pulse, which is called its bandwidth. The bandwidth depends on the shape and duration of a gradient pulse. Many shapes are possible for the gradient pulses used for selective excitation in MRI. The most widely cited gradient pulse shape is the sinc function. Sinc-shaped pulses excite an approximately rectangular distribution of spins, which is the ideal slice shape [1]. 


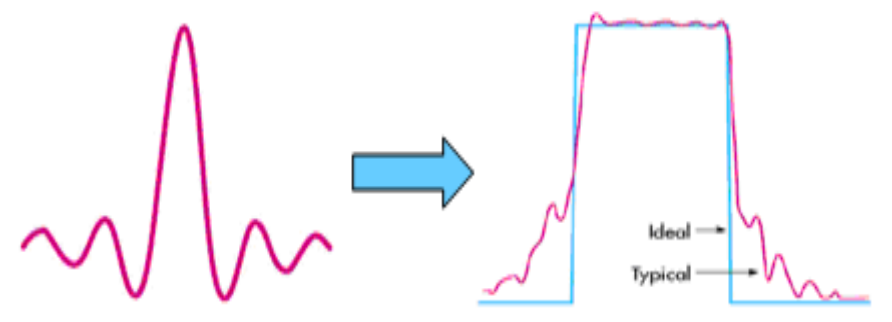

Figure 2.8 Common shape for envelope of slice-selective pulses in MRI which excite an approximately rectangular distribution of spins.

\subsubsection{Frequency Encoding}

Frequency encoding resolves spatial information along one direction of an MR image by keeping a magnetic field gradient on while each MR signal is being measured. The magnetic field gradient is called the frequency-encoding gradient, read-out gradient, or measurement gradient. The frequency-encoding gradient spreads the Larmor frequency over a range wide enough to distinguish 128 or 256 different locations along one direction. The Larmor frequency at the center of the FOV remains unchanged by the frequency-encoding gradient. However, the gradient increases the Larmor frequency on one side of the center and decreases it on the opposite side. As a result the frequency-encoding gradient affects the frequency of MR signals from tissues at different locations along the frequency-encoding direction. These signals are not detected separately. The frequency and phase of these MR signals are the important characteristics for identifying their location. That these signals also decay with time is of secondary importance here [1].

The frequency of each signal is unique to the location. An inverse FT of these complex k-space samples creates 13 pixels along the frequency-encoding direction of an MR image, which is purely real in this case. Only the frequency and phase of the signals are modeled here and not their decay or relative amplitude. By definition, lower-frequency signals take longer to complete each cycle, so more samples are 
acquired during each cycle [1].

\subsubsection{Phase Encoding}

Spatial information along one direction is encoded into the phase of MR signals by phase encoding. Phase is encoded into MR signals by pulsing a magnetic field gradient briefly ( $1 \mathrm{~ms}$ to $5 \mathrm{~ms}$ ) before each echo is sampled. The phase-encoding direction is perpendicular to the frequency-encoding direction. This is no different from the effect of the frequency-encoding gradient. While the phase-encoding gradient is on, the Larmor frequency becomes linearly proportional to the position along the phaseencoding direction. The key is the phase shift that has accumulated by the time the phase-encoding gradient pulse is turned off [5].

Phase shifts caused by the phase-encoding gradient depend on the location of the magnetization and the amplitude and duration of the phase-encoding gradient pulse. The phase-encoding gradient is pulsed to different amplitude before each MR signal so that the different rows of k-space can be filled. Stronger phase-encoding gradient pulses cause phase shifts. Doubling the amplitude doubles the phase shifts everywhere. The amount by which the phase changes as the phase-encoding gradient steps through its range of amplitudes is the key to identifying the location of structures along the phase-encoding direction [1].

\subsubsection{Two Dimensional Fourier transform Magnetic Reso- nance}

Fourier transformations (FT) decompose signals or curves into a distribution of cosine waves and sine waves of different frequency. The Fourier transformation evaluates the match between a curve and sinusoidal waves of a particular frequency $[1,6]$. 
The 2-DFT is used to sample the spatial frequency domain of the image on a rectangular grid. The 2-DFT technique consists of a basic cycle that is repeated many times, typically 256 or 512 . This cycle consists of an RF slice selection excitation pulse, a magnetic field pulse from the phase-encoding gradient, and then by a steady application of an orthogonal gradient that is the frequency-encoding gradient, during which time the MR signal is detected. After a suitable delay, the cycle is repeated. From one acquisition to the next, only the strength of the phase-encoding gradient is different in each cycle. The phase encoding gradient selects a single line in the spatial frequency domain representation of the image. Then the frequency-encoding gradient forms the MR signal along this line. By this way, the phase-encoding gradient shifts the MR signal so that it samples a different line parallel to the others (i.e. when the strength of the phase encoding gradient is changed in successive cycles, different lines in the spatial frequency domain are measured). A family of lines in the spatial frequency domain has been selected, and the frequency information along these lines has been measured with the phase-encoding gradient used to sample the frequency representation of the image in a rectangular coordinate system. This rectangular sampling is then Fourier transformed to yield the MR image [3,5].

\subsubsection{Sequence Timing}

Fourier imaging resolves spatial information by three distinct procedures, called selective excitation, phase encoding, and frequency encoding as shown in Figure[2.7]. Afterward, magnetization components everywhere in the slice regain the same Larmor frequency, but the phase depends on their position along the phase-encoding direction. Spatial information in the phase-encoding direction can be resolved if many

separate MR signals are collected. The amplitude of the phase-encoding gradient for each signal is decreased systematically. Each of these signals is measured as an 


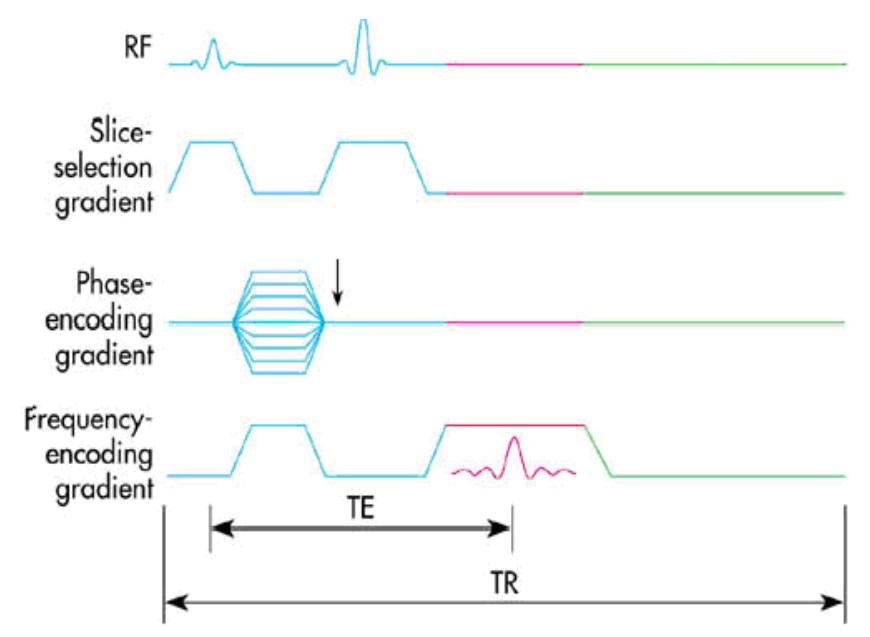

Figure 2.9 Pulse timing diagram for spin-echo pulse sequence in conventional Fourier.

echo while the frequency-encoding gradient is active, which creates a distribution of Larmor frequencies along the frequency-encoding direction. The first pulse of the frequency-encoding gradient is necessary for an echo to form during the middle of the second pulse [1].

\subsubsection{Repetition Time}

The time between repeated RF excitation pulses is called the repetition time (TR). The TR can be chosen from a certain minimum value, depending on the imaging technique and the MR system, to very long times. The time from the center of the $\mathrm{RF}$ excitation pulse to the center of the echo is the echo time (TE). The amplitude of the transverse magnetization at the echo peak depends on TE and $T_{2}$ of the tissue. In a spin echo this amplitude typically is proportional to $e^{-T E / T_{2}}$. [1,4] MR signals are sampled at equally spaced intervals of time. Continuous MR signals can be represented accurately by their samples if the interval between each sample is small enough (Figure (2.8)) [1].

The pulse sequence illustrated in Figure (2.7) must be repeated hundreds of times 


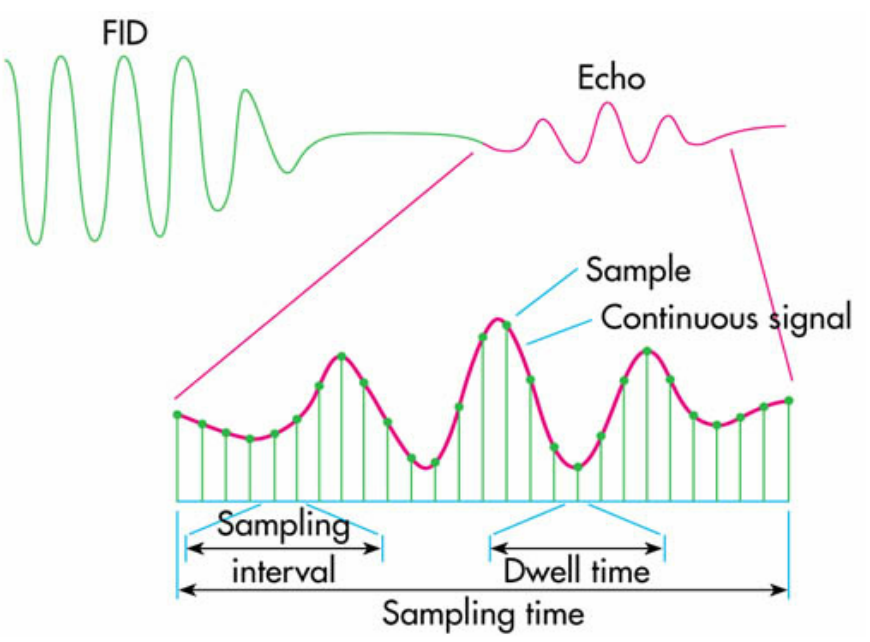

Figure 2.10 Representing difference between sampling time and sampling frequency.

to fill the $\mathrm{k}$ space for a $2 \mathrm{D} \mathrm{MR}$ image, depending on the number of pixels and redundant data for averaging or canceling various artifacts. Moreover, the sequence is usually executed initially for several seconds to allow the longitudinal magnetization to reach a steady state. The time for data acquisition is simply the product of TR and the number of pulse-sequence repetitions [1].

\subsubsection{K-space}

Images can be decomposed into thousands of sine and cosine waves of different frequency and phase. An array of numbers called k-space holds the weighting factor for each of these waves. The coordinates of k-space are called spatial frequencies, and their units are cycles per unit length. Each spatial frequency represents a sine or cosine wave across the entire image. The spatial frequencies $k_{x}$ and $k_{y}$ correspond to a 2D image with coordinates $\mathrm{x}$ and $\mathrm{y}[1,2]$. Each data sample in $\mathrm{k}$-space affects an entire MR image. An MR image acquires a pattern of lines if the data sample is displaced in either direction from the center of $\mathrm{k}$-space. The distance of the data sample from the center of $\mathrm{k}$ space determines the frequency of the repeated lines $[1,2]$. The center 


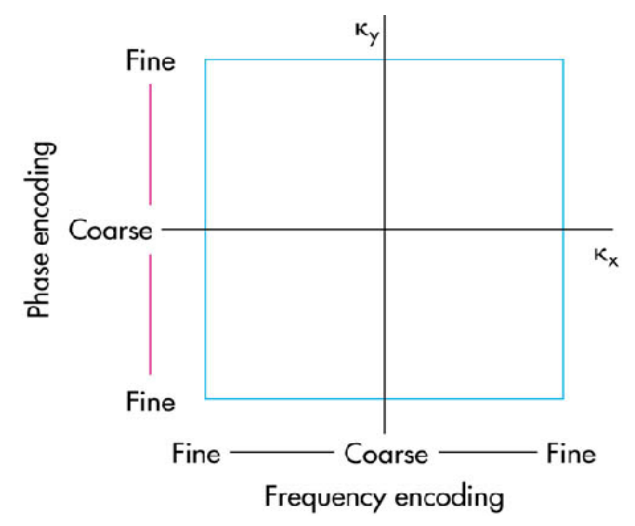

Figure 2.11 Influence of k-space regions on spatial properties.

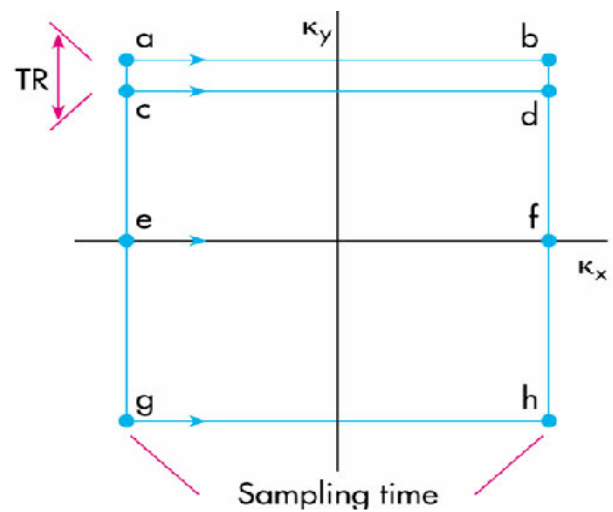

Figure 2.12 Difference between repetition time and Sampling time.

of k-space encodes coarse features in an image. Regions farther from center encode finer detail. Low and high spatial frequencies represent an object's overall shape and fine details, respectively. The transition from coarse structures to fine detail occurs gradually from the center of $\mathrm{k}$ space to its edges. An MR image produced from only low spatial frequencies is blurry. If k-space contains higher spatial frequencies, the associated image has higher spatial resolution [1].

\subsubsection{Image Reconstruction}

K-space contains complex numbers, which have real and imaginary parts. Both parts are passed to an inverse 2D DFT, as pictured in Figure2.13, and the output is a 


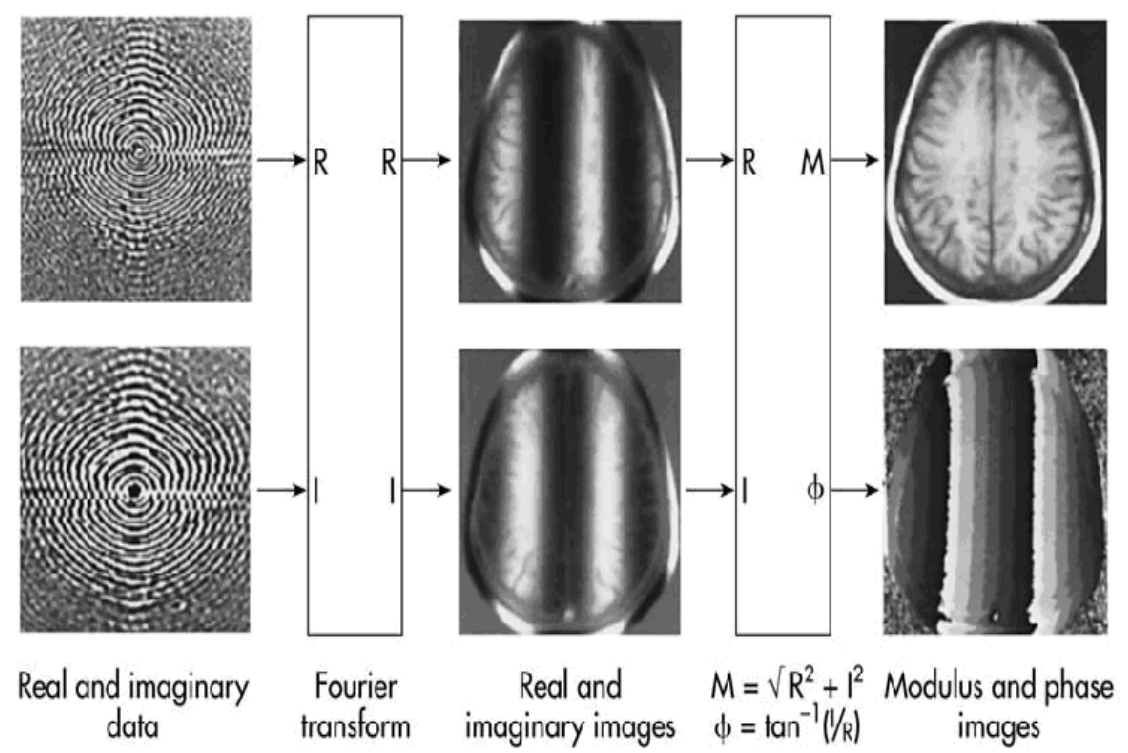

Figure 2.13 Reconstruction of 2D k space into 2D MR image.

matrix of complex numbers split into real and imaginary images. Usually, neither the real nor imaginary images are displayed because the image intensity is distributed indiscriminately between them. Instead the real and imaginary images are combined into a modulus (or magnitude) image. The modulus is appropriate for most MR images because pixels represent the magnitude of MR signals from that location [1].

\subsubsection{Image Contrast}

Adequate contrast among normal tissues is necessary for good anatomical definition. This adequate contrast between normal and diseased tissues is essential for sensitivity to disease [1].

The primary sources of tissue contrast in magnetic resonance imaging (MRI) are threefold-hydrogen spin densities $(\mathrm{N}[\mathrm{H}])$, longitudinal recovery times $\left(T_{1}\right)$, and transverse relaxation times $\left(T_{2}\right)$. Although hydrogen densities within soft tissues typically vary by only a few percent, the hydrogen contributing to the measured MR signal (referred to as the hydrogen spin density) tends to vary by a greater amount, up to 
$30 \%$ among soft tissues. $T_{1}$ and $T_{2}$ relaxation times often vary even more widely, sometimes varying among soft tissues by more than 100\%. Other sources of tissue contrast, such as flow, magnetic susceptibility inhomogeneities, and chemical shift have less noise component on the image contrast $[1,3-5]$.

\subsubsection{Pulse Sequence}

A pulse sequence is defined as a series of RF pulses, gradients applications and intervening time periods. by selecting the intervening time periods, image weighting is controlled. Pulse sequences are required because without a mechanism of refocusing spins, there is insufficient signal to produce an image. This is because dephasing occurs almost immediately after the RF excitation pulse has been removed. Spins lose their phase coherence in two ways:

- the increase of the intrinsic magnetic fields of adjacent nuclei.

- the inhomogeneities of the external magnetic field, i.e. some small areas of the field have a magnetic field strength of slightly more or less than the main field strength.

these steps are repeated many times, depending on the desired image quality. A wide variety of sequences are used in MR imaging based on the property that will be measured to differentiate between tissues.

\subsubsection{Image Noise}

Image noise is the primary deterrent to the discrimination of tissues and the detection of low-contrast lesions in MRI. There are two main categories of image noise: statistical and systematic [1]. 


\subsubsection{Statistical (or Random) Noise}

This type of noise is the pixel-to-pixel variation in signal intensities, apparent even for uniform tissues, caused by random signal fluctuations measured during signal sampling. At magnetic field strengths used for imaging, most statistical image noise is the result of eddy currents set up in the patient, producing spurious background signals that add to or subtract from true signals caused by precessing tissue magnetization. The results are random fluctuations in pixel intensities spread across the entire reconstructed image. Statistical noise is reduced relative to signal by increasing the voxel volume (slice thickness or in-plane pixel size), by increasing the number of acquisitions per phase-encoding step, by decreasing the sensitive volume of the receiver coil, or by decreasing the bandwidth, thereby narrowing the range of frequencies over which noise can be recorded $[1,6]$.

\subsubsection{Systematic Noise}

Except for extremely low SNR situations, systematic noise is usually more pervasive and confusing to image interpretation. Systematic noise consists of nonrandom signal variations that arise from a number of possible sources, including patient motion, such as respiration, vascular, and CSF pulsations; receiver-coil or gradient-coil motion; aliasing; and data truncation (Gibbs) artifacts. Although sometimes useful to the experienced radiologist, such systematic noise reveals itself as spurious signals in the image that do not reflect but tend to mask true inherent tissue properties [1].

\subsubsection{Safety and Bioeffects}

Death and injuries have occurred from projectiles created by the magnetic field, although compared to the millions of examinations administered. MRI makes use of 
powerful magnetic field which, though they have not been demonstrated to cause direct biological damage, can interfere with metallic and electromechanical devices. Additional(small) risks are presented by the radio frequency systems, components or elements of the MRI system's operation. The static magnetic field of MR scanner may be extremely strong with the field strengths commonly in the range of 1.5-4.0 T (15,000-40,000 gauss). Such strong magnetic field risks for both patients and personnel. A potential danger arises from ferromagnetic objects that may turn into dangerous missiles when brought near the magnet [4].

\subsubsection{MRI Modalities}

There are many MRI modalities capable of detecting a wide range of phenomena. Functional MRI (FMRI) measure signal changes in the brain due to neural activity changing. Diffusion Tensor MRI [DTMRI] measures the water molecule diffusion in the biological tissues. Perfusion MRI [PMRI] generates pictures of the arteries in order to evaluate them for stenosis, and spectroscopy, which is used to measure the levels of different metabolites in body tissues. In this work, we will be discussing Tensor Analysis and measurement that can be used in DTMRI images, which will be introduced in the Chapter 2.

\subsection{Diffusion Weighted and Diffusion Tensor MRI}

The basic principles of diffusion MRI were introduced in the mid-1980s; [1]. NMR imaging principles were used to encode molecular diffusion effects in the NMR signal by using bipolar magnetic field gradient pulses. Molecular diffusion refers to the random translational motion of molecules, also called Brownian motion, that results from the thermal energy carried by these molecules. The success of diffusion MRI 
is deeply rooted in the powerful concept that during their random motion, diffusion driven molecules probe tissue structure at a microscopic scale well beyond the usual image resolution [7].

The observation of this displacement distribution may thus provide unique clues to the structure and geometric organization of tissues $[7,8]$. MRI is the only means we have to observe diffusion in vivo noninvasively. Furthermore, MRI provides access to both superficial and deep organs with high resolution and does not interfere with the diffusion process itself. Diffusion is a three-dimensional intrinsic physical process that is totally independent of the MR effect or the magnetic field. This is not the case for most MRI-accessible parameters, such as $T_{1}$ or $T_{2}$ [7]. Hence, molecular mobility in tissues may not be the same in all directions. This anisotropy may result from a peculiar physical arrangement of the medium (such as in liquid crystals) or the presence of obstacles that limit molecular movement in some directions. As diffusion is encoded in the MRI signal by using magnetic field gradient pulses, only molecular displacements that occur along the direction of the gradient are visible. The effect of diffusion anisotropy can then easily be detected by observing variations in the diffusion measurements when the direction of the gradient pulses is changed. This is a unique, powerful feature not found with usual MRI parameters [7].

The measurement of self diffusivities of water and other solvents using the phenomenon of nuclear magnetic resonance (NMR) was first reported fifty years ago $[8,9]$. Methodological improvements in these diffusion measurements [10] and subsequent development of magnetic resonance imaging(MRI) together created the possibility to measure the diffusion properties in tissues on a voxel-by-voxel basis. [8]. In this chapter, historical background and theoretical concepts of diffusion-weighted imaging (DWI) and diffusion tensor imaging (DTI) as well as data analysis issues are treated with emphasis on DTI. We will review mathematical models underlying both DWI 
and DTI, and discuss quantitative parameters that are derived from the measured diffusion tensor.

\subsubsection{Diffusion Weighted Imaging (DWI)}

In 1828 the Scottish Naturalist Robert Brown published a pamphlet entitled 'A Brief account of microscopical observations ...' In which, Brown recorded that pollen grains of Clarkia pulchella suspended in water under a microscope exhibited a peculiar "rapid oscillatory motion" [11]. Brown initially believed that such motion was particular to the male sexual cells of plants, but was later startled to observe that pollen of plants suspended in alcohol for almost eleven months exhibited the same erratic motion: a "very unexpected fact of seeming vitality being retained by these 'molecules' so long after the death of the plant." Further studies with not only other

organic substances but chips of glass, granite, particles of smoke, and rocks "of all ages" revealed such motion to be a general property of small particles suspended in solution. The erratic particle motion observed by Brown would remain unexplained until the dawn of the kinetic theory of matter in the third quarter of the nineteenth century. Pioneered by Maxwell, Boltzmann, and Claussius, the kinetic theory of matter introduced the radical concept that the heat of a liquid or gas is mediated by the constant random thermal motion of the molecules in the medium. The kinetic theory would inspire Weiner to declare in 1863 that the particle motion observed by Brown could not be due to convection currents in the fluid, but was rather due to collisions between the particles and the surrounding molecules of the fluid. The molecular kinetic explanation for Brownian motion was reiterated some years later in 1877 by two Jesuit priests, Fathers Delsaulx and Carbonnelle, but it was not until G.L. Gouy in 1888-9 showed that Brownian motion is more rapid for smaller particles and never reaches equilibrium that the problem of Brownian motion assumed a place 
among the classical problems of physics. Albert Einstein, apparently unaware of any of the previous observations of Brownian motion, was motivated to provide an experimentally testable hypothesis for the kinetic-molecular theory of matter in 1905. He showed how the random thermal motion of the molecules in a liquid or gas could be imparted to larger particles. He predicted that the motion of these larger particles could be observed under the microscope $[12,13]$ and could therefore provide an experimentally testable hypothesis for the kinetic theory. Einstein's theory accounted for the dependence of the effect on the temperature and viscosity of the solution, and the size of the suspended particle, and thereby provided a set of experimentally testable predictions for the kinetic theory of matter [14]. Einstein's theoretical studies were experimentally confirmed by Jean Babtiste Perrin [15] who measured the dependence of Brownian motion on temperature and particle size. By demonstrating that colloidal particles obey Einstein's formulation, Perrin was able to calculate Avogadro's number and obtain direct verification for the kinetic-molecular theory of gases, a finding which earned him the Nobel Prize for physics in 1926. While Brownian motion is a microscopic phenomenon it gives rise to a macroscopically observable phenomenon known as diffusion. On a microscopic level, diffusion arises from the microscopic intermingling of the molecules as the result of Brownian motion. While we are familiar with the diffusion of a substance in another host substance, the physics of diffusion makes no essential distinction between the two. Hence, the theory of diffusion can be applied to the diffusion of a substance in its own medium, for example the diffusion of water in water. This phenomenon is referred to as self-diffusion or, with some abuse of terminology, simply diffusion.

Shortly after the initial discovery of the NMR phenomenon by Bloch $[16,17]$ and Purcell [18], Hahn published his seminal paper [19] on the NMR spin echo in which he noted that the random thermal motion of the spins would reduce the amplitude 
of the observed spin echo signal in the presence of a magnetic field inhomogeneity. Carr and Purcell [9] shortly after developed a set of equations for relating the echo amplitude attenuation to discrete jumps of the spins.

Torrey first incorporated anisotropic translational diffusion in the Bloch (magnetization transport) equations in 1956, which could lead to additional attenuation of the NMR signal $[17,18]$. Analytical solutions to this equation followed for freely diffusing species during a spin echo experiment [19] and, later, for diffusion in restricted geometries $[9,17,20]$. About a decade after its introduction, Stejskal and Tanner solved the Bloch-Torrey equation for the case of free, anisotropic diffusion in the principal frame of reference [10]

However, their formula is not generally usable to measure an effective diffusion tensor using NMR or MRI methods for several reasons. First, this formula relates a time-dependent diffusion tensor, to the NMR signal, so one must establish a relationship between the time-dependent diffusion tensor and an effective diffusion tensor. Second, in the pre-MRI era in which the formula was derived, it was always tacitly assumed that a homogeneous anisotropic sample could be physically reoriented within the magnet so that its principal axes could be aligned with the laboratory coordinate system [21].

After the development of MRI, This assumption was no longer tenable. Materials under study were often heterogeneous media whose 'fiber' or principal axes were generally not known a priori and could vary from place to place within the sample. Thus, a general scheme had to be developed to measure the entire diffusion tensor (both its diagonal and off-diagonal elements) in the laboratory frame of reference [21]. In Diffusion Imaging (DI), one measures a single scalar apparent diffusion constant (ADC) in each voxel from a series of diffusion weighted images (DWIs). These are just conventional MRIs whose contrast is sensitized or weighted by the local diffusivity in 
each voxel $[8,10,22]$.

The effect of diffusion on the MRI signal (most often a spin-echo signal) is an attenuation, A, which depends on ADC in the voxel and on a scalar b- factor, which characterizes the gradient pulses (timing, amplitude, shape) used in the MRI sequence [8].

The b-factor can easily be calculated analytically for simple pulse sequences used in spectroscopy. However, complicated imaging pulse sequences may contain localization. B-factors can be evaluated either numerically or experimentally (by calibrating the diffusion coefficient from a phantom material). Accurate determination of the b-factor, taking into account all gradient pulses, is necessary for diffusion measurements [23].

The Diffusion Weighted Imaging (DWI) is used to measure the translational mobility of diffusing molecules along one direction which depends upon a medium's orientation. The diffusion is anisotropic in biological tissues such as brain white matter, skeletal muscle, kidney and cardiac muscle. The ADC depends on the choice of laboratory coordinate system. But in other tissues, such as the gray matter and the cerebrospinal fluid, the diffusion is isotropic; the ADC is independent of the orientation of the tissue relative to the laboratory frame of reference $[7,24,25]$.

From these DWIs, we can estimate the ADC in each voxel using linear regression:

$$
A=\frac{S(b)}{S(b=0)}=\exp ^{-b A D C}
$$

where $S(b)$ and $S(b=0)$ are the echo magnitudes of the diffusion weighted (after application of b-diffusion application of b-diffusion gradient) and non-diffusion weighted signals (without any applied diffusion gradient)respectively.[4]

$$
\ln \left(\frac{S(b)}{S(b=0)}\right)=-b A D C
$$

One complication is that imaging and diffusion gradients interact with one another, 
producing additional cross terms in the b factor that can lead to an incorrect estimation of ADC [24].

\subsubsection{Anisotropy in DWI}

Diffusion isotropy describes the case in which the translational mobility of the diffusing molecule is independent of the medium's orientation. Homogeneous diffusion refers to the case in which the translational mobility of the diffusing molecule is independent of the position within the medium. If a medium is both isotropic and homogeneous, the mobility will be independent on neither the gradient direction nor the medium orientation [7].

Several different scalar indices derived from diffusion weighted images (DWls) have been used to characterize diffusion anisotropy [24]. Moseley et al [26] characterized diffusion anisotropy in each voxel by the ratio of differences and sums of DWls with diffusion-sensitizing gradients applied in two perpendicular directions. e.g. $\mathrm{x}$ and $\mathrm{y}$ :

$$
\frac{D W I_{x}-D W I_{y}}{D W I_{x}+D W I_{y}}
$$

Douek et al. [27] characterized by the ratio of two apparent diffusion constants (ADCs), measured with diffusion-sensitizing gradient applied in two perpendicular directions. e.g. $\mathrm{x}$ and $\mathrm{y}$ :

$$
\frac{A D C_{x}}{A D C_{y}}
$$

and displayed as a color image. In voxels containing one particular tissue (such as white matter) this ratio was a maximum. Its value was assumed to be $A D C_{\perp} / A D C_{\|}$, $[25,27]$ the ratio of ADCs perpendicular to and parallel to the fiber tract direction. Recently, van Gelderen et al. [28] proposed a scalar anisotropy index that is proportional to the standard deviation of three ADCs measured in three mutually perpendicular 
directions: $A D C_{x}, A D C_{y}$, and $A D C_{z}$, divided by their mean value (ADC) [24,29].

$$
\frac{\left(A D C_{x}-\langle A D C\rangle\right)^{2}+\left(A D C_{y}-\langle A D C\rangle\right)^{2}+\left(A D C_{z}-\langle A D C\rangle\right)^{2}}{\langle A D C\rangle}
$$

where

$$
\langle A D C\rangle=\frac{A D C_{x}+A D C_{y}+A D C_{z}}{3}
$$

Unfortunately, none of these anisotropy measures is rotationally invariant. Anisotropy measures based upon DWls are inherently nonobjective; that is, their contrast does not correspond to a single meaningful physical or chemical variable or fundamental parameter, but to a complicated combination of them. This is usually true for anisotropy measures that use the ADC, since they are estimated from DWls using a model that may assume diffusion is isotropic. Even so, these anisotropy measures suffer from a more serious failing, They inherently depend on [7]:

- The choice of the laboratory frame of reference (i.e., the $\mathrm{x}, \mathrm{y}, \mathrm{z}$ coordinate system used to represent the directions of the static $B_{0}$ field and the applied magnetic field gradients in an MR experiment);

- The choice of the direction of the applied diffusion gradients used to acquire the DWIs;

- The orientation and placement of the sample within the magnet; and

- The orientation and position of the macromolecular, cellular, and/or fibrous structures within a voxel that produce the observed diffusion anisotropy.

Clearly, for an anisotropy index (or any other scalar measure of an intrinsic characteristic or feature) to possess the properties of a quantitative histological stain (such as an autoradiography,) it should be objective (i.e., its value in each voxel should be a known monotonic function of a meaningful physical quantity) and it should be invariant with respect to arbitrary rotations and translations. These intuitive criteria are 
used explicitly below to constrain the set of admissible scalar measures of structural features (such as diffusion anisotropy, structural similarity, and fiber organization) that we derive from the diffusion tensor [7].

These indices are so-called rotationally variant indices that depend on the direction of the applied diffusion gradient direction and the tissue orientation within each voxel. More recently other indices derived from DTI have been developed [7, 24,30-32], we will review some of these.

\subsubsection{Applications of DWI}

The practical use of DWI became clear when it was noted that water apparent diffusion is significantly slower in regions of ischemia compared with normal regions of cat and rat brain, with ADC decreasing by $30 \%$ to $60 \%$ after the onset of stroke [7]. These regional differences in ADC (apparent as hyper intensity in DW images) are correlated with total or near-total perfusion deficits; the ADC does not decrease until cerebral blood flow $(\mathrm{CBF})$ drops below 15 to $20 \mathrm{ml} / 100 \mathrm{~g} / \mathrm{min}$. Drops in the ADC are not readily observed in partially reduced flow states. This finding strongly suggests that when collateral blood flow is sufficient to prevent the breakdown of cellular metabolism, the ADC does not drop. The observation that the ADC is coupled to metabolic processes is reinforced by recent reports of ADC decreases observed in models of status epilepticus and spreading depression which can not be seen on

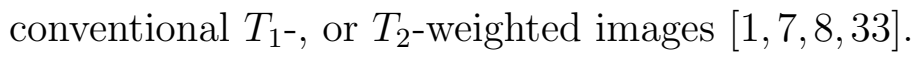

\subsubsection{Diffusion Tensor Imaging}

Since the DWI is inherently a one-dimensional technique, i.e. it can only meaningfully measure molecular displacements along one direction, it can be enough to describe the diffusivity of an isotropic material such as gray matter. In such tissues, where the 
measured apparent diffusivity is largely independent of the orientation of the tissue, it is usually sufficient to characterize the diffusion characteristics with a single (scalar) apparent diffusion coefficient (ADC). However, in anisotropic media, it is not enough for full characterization of an anisotropic tissue such as the white matter which needs a three dimensional description [7,24]. The measured diffusivity is known to depend upon the orientation of the tissue [8].

Diffusion tensor imaging (DTI) [25] was developed to describe diffusion in an anisotropic medium [7], Scalar coeffecisnt are cannot describe the complex anisotropic property diffusion behavior [34]. An effective diffusion tensor D can be estimated from DWls using a more general relationship between the measured echo magnitude in each voxel and the applied magnetic field gradient sequence $[8,34]$.

Furthermore, diffusion is truly a three-dimensional process. Hence, molecular mobility in tissues may not be the same in all directions. This anisotropy may result from a peculiar physical arrangement of the medium (such as in liquid crystals) or the presence of obstacles that limit molecular movement in some directions. However, in the presence of anisotropy, diffusion can no longer be characterized by a single scalar coefficient, but requires a tensor, D, which fully describes molecular mobility along each direction and correlation between these directions [8].

As diffusion is encoded in the MRI signal by using magnetic field gradient pulses, only molecular displacements that occur along the direction of the gradient are visible. The effect of diffusion anisotropy can then easily be detected by observing variations in the diffusion measurements when the direction of the gradient pulses is changed [8].

\subsubsection{Pulsed Gradient Echo Pulse Sequence}

To determine the diffusion tensor fully, diffusion-weighted images must be collected along several gradient directions, using diffusion-sensitized MRI pulse sequences such 


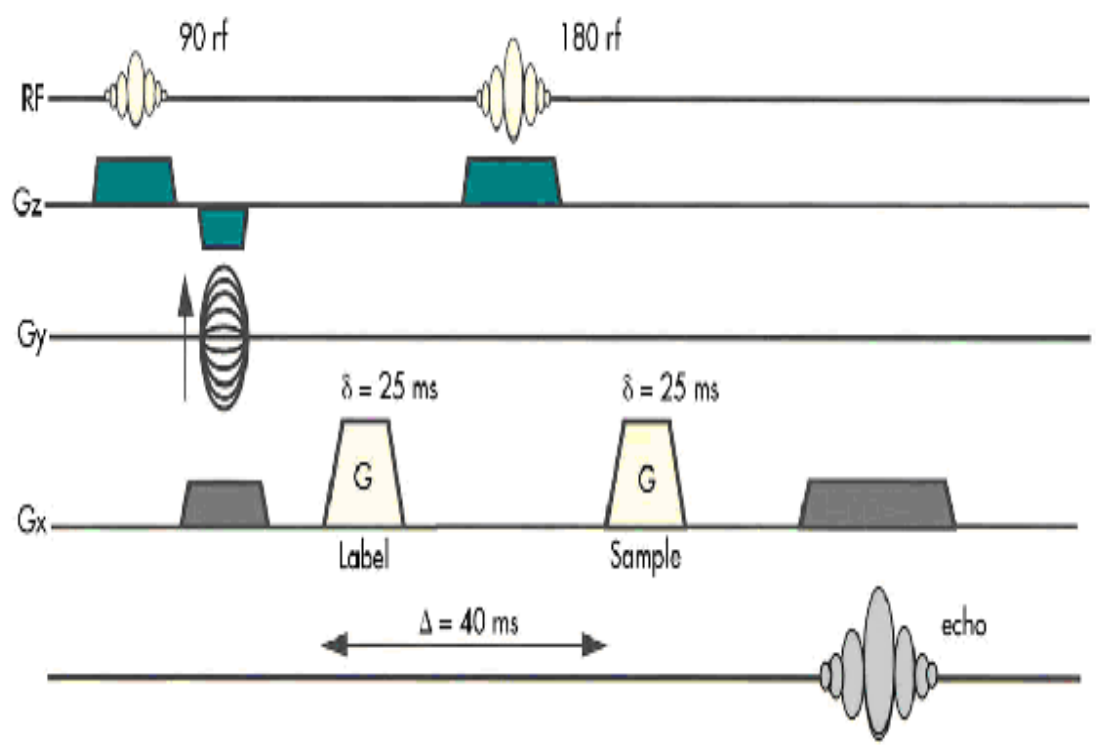

Figure 2.14 Timing diagram for ST SE DW pulse sequences and series of DW images of the human brain.

as echo planar imaging (EPI) [8].

In single tensor estimation, the diffusion tensor is symmetric, measurements along at least six directions are mandatory, along with an image acquired without diffusion weighting $(b=0)$. The diffusion is observable as a shortening on the spin-echo (SE). This signal has prompted the development of numerous nuclear MR methods to measure the self-diffusion coefficient of liquids; the most commonly used method is the pulsed magnetic field gradient SE technique of Stejskal and Tanner. The StejskalTanner (ST) method applies a pair of identical square gradient pulses of strength G (expressed in Gauss per centimeter or in milliTesla per meter) to an SE pulse sequence, thus making the measurement quantitative and more sensitive to diffusion. A major advantage in the pulsed gradient approach is that the stronger gradient pulses overcome poor local magnetic field homogeneities, which are found particularly in vivo tissues [8]. The ST SE sequence has advantages in that the time during which the sequence is sensitive to diffusion $(\delta-\Delta / 3)$ can be accurately controlled. From 


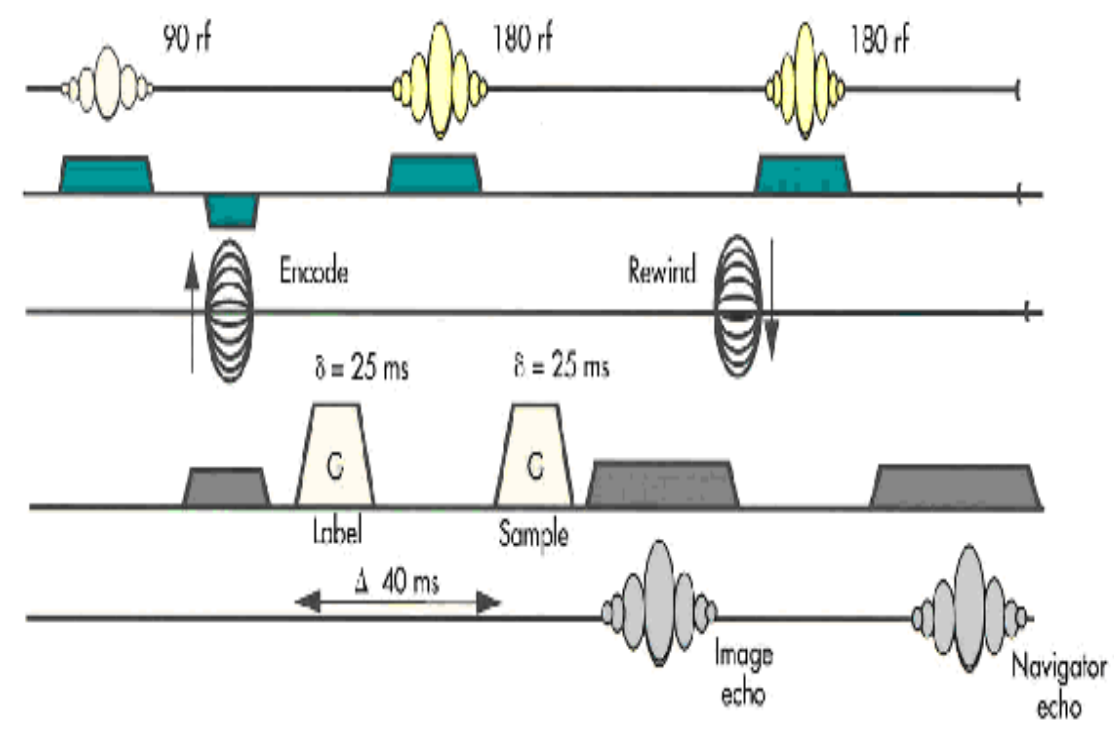

Figure 2.15 Principles of Navigation in DWIs.

the observation time and the measured diffusion coefficient, restriction of diffusion can be evaluated. Also, the direction of the applied diffusion-sensitizing gradient in the ST pulse sequence can be chosen by using one of the $\mathrm{x}-$, y-, or z-axis magnetic field gradients, which allows assessment of the tissue diffusional anisotropy tensor (differing diffusional rates along different directions). In addition, DW images can be acquired with the anisotropic effect averaged out (by mapping the trace of the tensor) or enhanced (by mapping the tensor deviation) [1,22]. However, motion artifacts have severely limited the ability of this approach. To correct for interview phase variations, a navigational motion-correction scheme uses an extra SE in the sequence, in which the added navigator echo has no spatial phase encoding. Motioninduced phase and magnitude variations in the second navigator echo are then used to correct the phase errors occurring in the first imaging echo before two-dimensional (2D) Fourier transformation (FT). One-directional (1D) navigated DW techniques can significantly reduce the motion sensitivity present in conventional SE [1].

A typical set of gradient combinations that preserves uniform space sampling and 

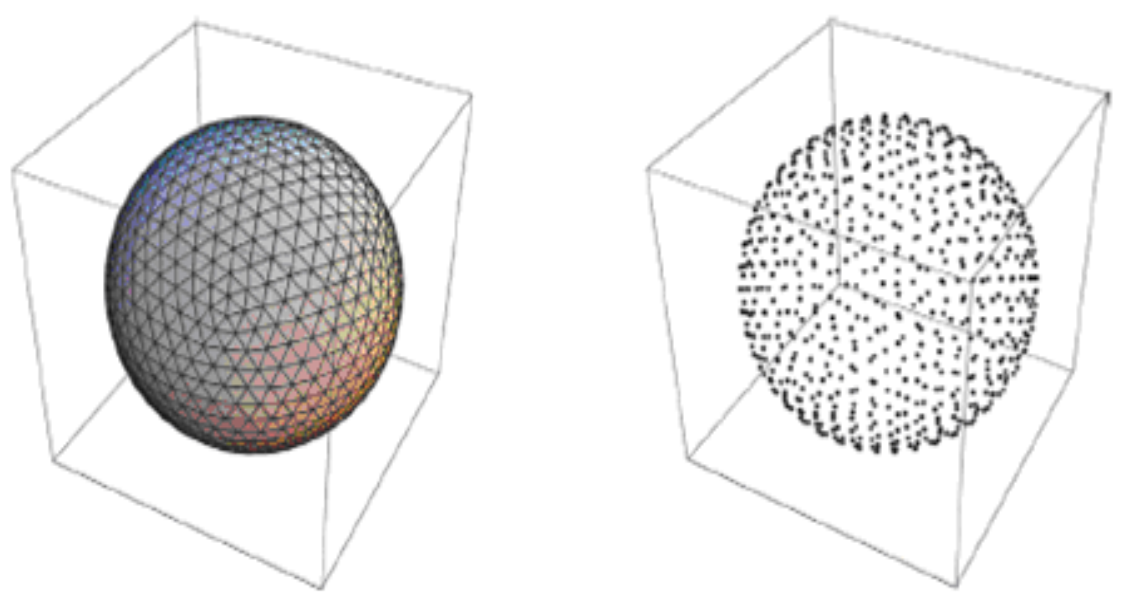

Figure 2.16 Diffusion-encoding directions generated by the pulse sequence are spherical tessellations of an icosahedron.

similar b-factor along each direction is as follows (coefficients for gradient pulses along the $(\mathrm{x}, \mathrm{y}, \mathrm{z})$ axes, normalized to a given amplitude, $\mathrm{G}) \cdot\left(\frac{1}{\sqrt{(2)}}, 0, \frac{1}{\sqrt{(2)}}\right)$ : $\left(-\frac{1}{\sqrt{(2)}}, 0, \frac{1}{\sqrt{(2)}}\right):\left(0, \frac{1}{\sqrt{(2)}}, \frac{1}{\sqrt{(2)}}\right):\left(0, \frac{1}{\sqrt{(2)}},-\frac{1}{\sqrt{(2)}}\right):\left(\frac{1}{\sqrt{(2)}}, \frac{1}{\sqrt{(2)}}, 0\right):\left(-\frac{1}{\sqrt{(2)}}, \frac{1}{\sqrt{(2)}}, 0\right)$ This minimal set of images may be repeated for averaging, to increase the signal to noise ratio (SNR). [8] Efforts are now being made to collect data along as many directions in space as possible to avoid sampling direction biases. This uniform space sampling paradigm is particularly interesting for fiber tracking applications and provides a gain in SNR $[8,35]$

High Angular Resolution Diffusion (HARD) encoding was achieved by generating gradient directions equally spaced on a sphere by tessellations of an icosahedron [36, 37]. The icosahedron uniform space sampling is a widely used scheme for directional sampling [34], as shown in Figure (2.16). This procedure produces directions that are equally separated in angle on the surface of a sphere [38]. 


\subsubsection{Diffusion Tensor Reconstruction}

The diffusion tensor is a mathematically elegant description of diffusion as a function of direction [39] It is fully describes the molecular mobility along each direction (x, $\mathrm{y}, \mathrm{z})$ and the correlation between these directions [34]. The measurement of D in each voxel and the analysis and the display of information derived from it is called Diffusion Tensor Imaging (DTI) [7,40].

Diffusion anisotropy in white matter originates from its specific organization in bundles of more or less myelinated axonal fibers running in parallel, although the exact mechanism is still not completely understood: diffusion in the direction of the fibers is faster than in the perpendicular directions. It quickly appeared that this feature could be exploited to map out the orientation in space of the white matter tracts in the brain using a color scale, assuming that the direction of the fastest diffusion would indicate the overall orientation of the fibers $[8,27,41]$.

\subsubsection{Diffusion Tensor Eigen System}

The diffusion tensor $\mathrm{D}$ is a mathematical description of diffusion as a function of the direction and for uncharged molecules such as water in the human body, D is symmetric. [42]

$$
\mathrm{D}=\left[\begin{array}{ccc}
D_{x x} & D_{x y} & D_{x z} \\
D_{x y} & D_{y y} & D_{y z} \\
D_{x z} & D_{y z} & D_{z z}
\end{array}\right]
$$

In DTI one uses a set of DWIs and their corresponding scalar b-factors to estimate an ADC along a particular direction using linear regression [8,34]. 


$$
\ln \left(\frac{S(b)}{s(b=0)}\right)=-\sum_{i=1}^{3} \sum_{j=1}^{3} b_{i j} D_{i j}
$$

Another step is to determine the main direction of diffusivities in each voxel and the diffusion values associated with these directions by diagonalization of the diffusion tensor to provide eigen-vectors $v_{1}, v_{2}, v_{3}$ and eigen-values $\lambda_{1}, \lambda_{2}, \lambda_{3}$ which correspond respectively to the main diffusion directions and associated diffusivities - These eigen diffusivities represent the unidimensional diffusion coefficients in the main eigen-vectors directions of diffusivities of the medium.

\subsubsection{Diffusion in an Isotropic Medium}

Diffusion isotropy describes the case in which the translational mobility of the diffusing molecule is independent of the medium's orientation. Homogeneous diffusion refers to the case in which the translational mobility of the diffusing molecule is independent of the position within the medium. $\left(\left(D_{x x}=D_{y y}=D_{z z}\right)[8,32]\right.$ or $\left.\left(\lambda_{1}=\lambda_{2}=\lambda_{3}\right)\right)$ If a medium is both isotropic and homogeneous, then the translational displacement profile is given by: $[7,39,42]$

$$
\rho\left(r \mid \tau_{d}\right)=\frac{1}{\sqrt{\left(4 \pi D \tau_{d}\right)}} \exp \left(-\frac{r^{T} r}{4 \pi D \tau_{d}}\right)=\frac{1}{\sqrt{\left(4 \pi D \tau_{d}\right)}} \exp \left(-\frac{x^{2}+y^{2}+z^{2}}{4 D \tau_{d}}\right)
$$

\subsubsection{Diffusion in an Anisotropic Medium}

In biological tissues such as brain white matter, we can ascribe anisotropic diffusion (observed in MR spectroscopy or imaging studies) to spatial variations of molecular mobility (heterogeneity) at micron scales. This phenomenon appears to be caused primarily by the spatial arrangement of macromolecular, membranous, and fibrous constituents and their interfaces. In such tissues, diffusion anisotropy can be charac- 
terized within a macroscopic voxel by an effective diffusion tensor, $\underline{D}[32,43]$ :

$$
\rho\left(r \mid \tau_{d}\right)=\frac{1}{\sqrt{|\underline{D}|\left(4 \pi \tau_{d}\right)}} \exp \left(\frac{-r^{T} \underline{D} r}{4 \tau_{d}}\right)
$$

Clearly, in an anisotropic medium, the six independent components of the symmetric tensor are required to describe the three dimensional displacements of particles, whereas in an isotropic medium, only one parameter, D, is sufficient. These additional parameters are required because in anisotropic media, displacements generally appear to be correlated in both parallel and perpendicular directions, whereas in isotropic media they do not. In fact, the elements of the diffusion tensor represent the magnitude of the correlations between the translational displacements in parallel and perpendicular directions. Specifically, the diagonal elements of D, $D_{x x}, D_{y y}$, and $D_{z z}$ represent the strength of correlations between molecular displacements along the same directions (i.e., along x, y, and z. respectively), while its off-diagonal elements, $D_{x y}, D_{x z}, D_{y z}$, represent strength of correlations in molecular displacements along perpendicular directions (i.e., between $\mathrm{x}$ and $\mathrm{y}, \mathrm{x}$ and $\mathrm{z}$. and $\mathrm{y}$ and z. respectively). In anisotropic media the diagonal elements of the diffusion tensor are generally unequal, whereas in isotropic media they are all equal. Moreover, in anisotropic media the off-diagonal elements are generally non-zero and may be large (i.e., comparable in magnitude to the diagonal elements), whereas in isotropic media they all equal zero [43].

\subsubsection{Quantitative Parameters Obtained by DT MRI}

Quantitative parameters provided by diffusion-tensor MRI can be obtained and explained using a geometric approach. Intrinsic quantities can be used that characterize different unique features, for example, describing the size, shape and orientation of the root mean square (rms) displacement profiles within an imaging volume, which 
can be represented as diffusion ellipsoids. Scalar parameters, functionally related to the diagonal and offdiagonal elements of tensor $D(x, y, z)$, can also be displayed as an image, revealing ways in which the tensor field varies from place to place within the imaging volume. These quantities are rotationally invariant, i.e. independent of the orientation of the tissue structures, the patient's body within the MR magnet, the applied diffusion sensitizing gradients, and the choice of the laboratory coordinate system in which the components of the diffusion tensor [7,8,25].

Several invariant indices are thus made of combinations of the terms of the diagonalized diffusion tensor, i.e., the eigen-values $\lambda_{1}, \lambda_{2}$ and $\lambda_{3}$. The most commonly used invariant indices are the fractional anisotropy (FA), the relative anisotropy (RA), and the volume ratio (VR) indices, defined respectively as [43]:

1. Fractional Anisotropy (FA): measures the fraction of the magnitude of $\mathrm{D}$ that can be ascribed to anisotropic diffusion. It varies between 0 (isotropic diffusion) and 1 (infinite anisotropy).

$$
F A=\frac{\sqrt{3\left[\left(\lambda_{1}-\bar{\lambda}\right)^{2}+\left(\lambda_{2}-\bar{\lambda}\right)^{2}+\left(\lambda_{3}-\bar{\lambda}\right)^{2}\right]}}{\sqrt{2\left(\lambda_{1}^{2}+\lambda_{2}^{2}+\lambda_{3}^{2}\right)}}
$$

2. Relative Anisotropy (RA):is a normalized standard deviation also represents the ratio of the anisotropic part of $\mathrm{D}$ to its isotropic part. It varies between 0 (isotropic diffusion) and $\sqrt{2}$ (infinite anisotropy).

$$
R A=\frac{\sqrt{\left[\left(\lambda_{1}-\bar{\lambda}\right)^{2}+\left(\lambda_{2}-\bar{\lambda}\right)^{2}+\left(\lambda_{2}-\bar{\lambda}\right)^{2}\right]}}{3 \bar{\lambda}}
$$

3. Volume Ratio (VR): represents the ratio of the ellipsoid volume to the volume of a sphere of radius l. Its range is from 1 (isotropic diffusion) to 0(infinite anisotropy), Since it is the opposite meaning of the FA and RA, some authors prefer to use (1-VR).

$$
V R=\frac{\lambda_{1} \lambda_{2} \lambda_{3}}{\overline{\lambda^{3}}}
$$




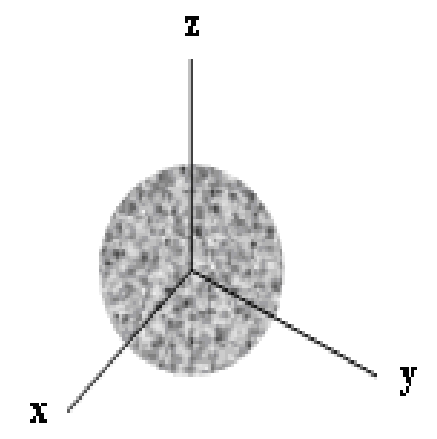

Figure 2.17 Homogenous isotropic tissue diffusion profile.

where $\bar{\lambda}$ is the mean diffusivity:

$$
\bar{\lambda}=\frac{\lambda_{1}+\lambda_{2}+\lambda_{3}}{3}
$$

\subsubsection{Diffusion Profiles of Various Brain Tissues}

There are three main categories into which the human brain tissue can be classified to:

(a) Cerebro-spinal fluid (CSF): located around the brain and the ventricles. Its diffusion profile is much like that of unconstrained diffusing water as it is a homogeneous isotropic tissue. Its principal eigen-diffusivities (eigenvalues) would be related such that: $\lambda_{1}=\lambda_{2}=\lambda_{3}$.

(b) Gray matter (GM): constituting the brain cortex with its nerved cell bodies and nerve centers. The relation between its principal eigen-diffusivities is such that: $\lambda_{1} \approx \lambda_{2} \approx \lambda_{3}$

(c) White matter (WM): compromising the main nerve fiber bundles in the brain. Its principal eigen diffusivities would have the typical relation of: $\lambda_{1}>>\lambda_{2} \approx \lambda_{3}$.

Then, the corresponding ellipsoid would be of cigar-shape [23]. This shape is predominantly observed in white matter fibers, especially in the corpus callosum and in the pyramidal tract in monkeys and in humans. 


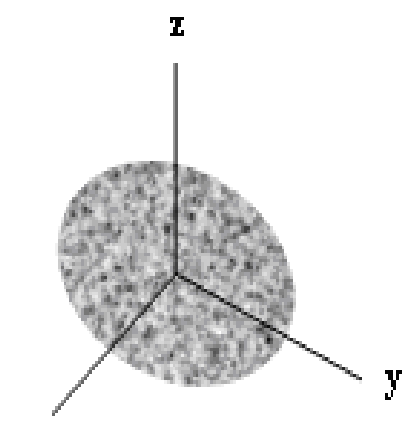

$\mathbf{x}$

Figure 2.18 Homogenous isotropic tissue diffusion profile.

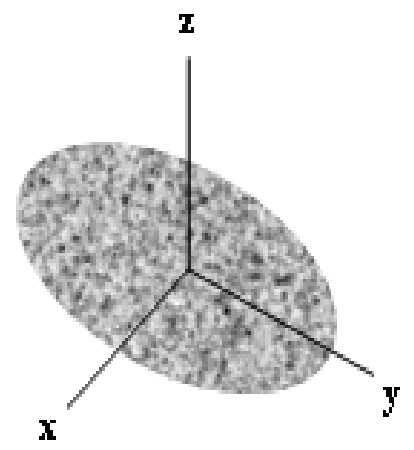

Figure 2.19 Anisotropic white matter diffusion profile. 


\section{Chapter 3}

\section{Multi- and Higher Rank Tensors}

\subsection{Introduction}

Diffusion-tensor imaging (DTI) has long been touted as a means to chart the macroscopic connectivity network of the human brain that is, a means to piece together a circuit diagram of sorts for human neuroanatomy [8]. The microscopic neuronal/axonal scale in the human brain is beyond the limited resolution of DTI, and moreover, the sheer magnitude of a $10^{11} \times 10^{11}$ adjacency matrix comprising in the order of $10^{15}$ nonzero elements is unmanageable. At the macroscopic scale, the basic unit of connectivity are fiber bundles, each of which comprise in the order of $10^{3}-10^{5}$ closely packed axons following a common trajectory in the sub-cortex. Fiber bundles serve as long-distance pathways between distinct regions located in the cortex. Connectivity at the macroscopic scale is approximated with a network (graph) model, where distinct cortical regions play the role of nodes (vertices), while fiber bundles form the interconnecting links (edges). [44]

In this chapter, we will introduce the meaning of multi tensors and higher rank tensors. 


\subsection{Multi Tensors}

Several authors reported the non-mono-exponential behavior for the diffusion-induced attenuation in brain tissue in water and NAA signals. Their studies were based on a 1-D diffusion experiment and the results were fitted to bi- or tri-exponential functions. They indicated that high b values reveal more complicated behavior and therefore their fit depended on the value of b used in the measurement.

In order to overcome the regular tensor model limitations, higher order tensors [45] were introduced to represent more complicated diffusivity profiles which better approximate the local diffusivity function. Generalized scalar quantities such as the variance of diffusivity and the generalized anisotropy were derived as functions of the higher order tensor coefficients [46]. However, in all these works the higherorder tensors are estimated without imposing the positivity of the diffusivity function approximation, which is significantly important since negative diffusivity values are non physical.

Basser et al. [34] discussed the possibility of mixture modeling of diffusion. Even though they indicated that this would present a more complete representation of the process, they argued that there too many issues that need to be resolved before such modeling can be performed in practice. In particular, their hypothetical discussion indicated that such modeling would require a large amount of data to enable the estimation of model parameters and involve the computation of too many parameters.

They suggested also that several problems had to be addressed in such experiments that included optimization of diffusion gradient directions and model order selection. They concluded that this area had many aspects that were yet to be investigated.

Hsu et al. [47] proposed a two-compartment model for the diffusion in fibers of the myocardium. They reported two fast and slow components in their study while 
assuming a slow-exchange process between the two. Inglis et al. [48] reported biexponential diffusion tensor measurements. They hypothesized that these components may represent the intra- and extra-cellular components in tissues. Clark et al. [49] reported variations of the apparent diffusion coefficient with the value of diffusion time. Their hypothesis was that such variations are important indicators of restricted flow, which present a potentially large diagnostic value. In a later study by the same group, Clark et al. [50] reported results of a two-tensor model for diffusion in the human brain. They measured the parameters of a mixture model composed of two weighted tensors. Their results indicated the presence of fast and slow diffusion components, and that each can be modeled by a unique tensor. They indicated that the use of high b-factor was essential to reveal the slow component of diffusion.

Spherical harmonics $(\mathrm{SH})$ are used to solve a large variety of physical problems. Frank [51] used SH in diffusion tensor for displaying the apparent diffusion in free space.

He pointed out that the shape of the surface of the measured diffusion along multiple directions for a single voxel in high angular resolution diffusion measurements can convey more information than the diffusion tensor. This idea leads to the identification of diffusion anisotropy by using the variations of $D_{a p p}$ from a sphere without the necessity of invoking the diffusion tensor formalism. [5] The original model for tensor representation become insufficient to describe the whole voxel fibers. Higher order tensors are more relvant to describe more precisely the voxels' fiber content.

A number of methods has been used to calculate multi tensors, assumed to have 2 compartments, one isotropic and one anisotropic. this model requires low b-factor and high b-factor images for estimation. Tuch et al. [52] assumes having 2 fibers of known anisotropicity. and the target was to calculate the angle between them. Kadah et al. [53] used the differentiation and the exhaustive search to calculate these 
tensors. The main disadvantage of such algorithms are the number of compartment assumption, and the high mathematical and processing complexity of the algorithms.

\subsection{Higher Order Tensors}

To provide a formal basis for the modeling of highly structured diffusion, an extension to the Bloch- Torrey equation [20] was proposed to include a phenomenological diffusion term with a high-rank Cartesian tensor, which yields a generalized StejskalTanner formula will be given by Equation [3.1]

$$
\begin{gathered}
\ln (s)=\ln \left(S_{0}\right)-b \sum_{i_{1}=1}^{3} \sum_{i_{2}=1}^{3} \ldots \sum_{i_{l}=1}^{3} D_{i_{1} i_{2} \ldots i_{l}} g_{\left(i_{1}\right)} g_{\left(i_{2}\right)} \ldots g_{\left(i_{l}\right)} \\
\left.\left.D(g)=\sum_{i_{1}=1}^{3} \sum_{i_{2}=1}^{3} \ldots \sum_{i_{l}=1}^{3} D_{i_{1} i_{2} \ldots i_{l}} g_{(} i_{1}\right) g_{(} i_{2}\right) \ldots g\left(i_{l}\right) \\
D(-g)=-D(g)
\end{gathered}
$$

This equation makes it possible to calculate all the components of the DT of general rank by means of a simple multilinear regression. However, since negative diffusion coefficients are nonphysical, the tensor rank is forced to be an even number.

A general rank-1 Cartesian tensor has $3^{l}$ components, which is a very large number for higher ranks. For example, a rank-10 tensor will have 59049 components. However, symmetries provide a very significant reduction in the number of distinct components. This follows from the realization that $D_{i_{1} i_{2} \ldots i_{l}}$ is a totally symmetric tensor. Total symmetry is due to the fact that this tensor links the components of the same vector to a scalar $(\mathrm{D}(\mathrm{g}))$.

$$
D_{i_{1} i_{2} \ldots i_{l}}=D_{\left(i_{1} i_{2} \ldots i_{l}\right)}
$$

where $\left(i_{1} i_{2} \ldots i_{l}\right)$ stands for all permutations of the indices. This symmetry reduces the 
number of distinct elements to

$$
N_{l}=\frac{(l+1)(l+2)}{2}
$$

which is only 66 for $l=10$ case. To use Equation [3.1] to derive the distinct components of the rank-1 DT, the number of times a given element is repeated is in need to be known. The multiplicity of that element will be denoted with the letter $\mu$. Knowing the multiplicity of every unique element, Equation [3.1] can be rewritten as:

$$
\ln (s)=\ln \left(S_{0}\right)-b \sum_{k=1}^{N_{l}} \mu_{k} D_{k} \prod_{p=1}^{l} g_{k(p)}
$$

where $D_{k}$ is the $k^{t h}$ unique element of the tensor, and $g_{k(p)}$ is the component of the gradient direction specified by the $p^{\text {th }}$ index of the $k^{\text {th }}$ unique element of the generalized DT. The multiplicity of a component of a rank-l tensor is given by

$$
\mu=\frac{l !}{n_{x} ! n_{y} ! n_{z} !}
$$

where $n_{x}, n_{y}$, and $n_{z}$ are respectively the number of the $\mathrm{x}, \mathrm{y}$, and $\mathrm{z}$ indices included in the full sequence of subscripts defining the component of the tensor [45].

\subsubsection{Generalized Anisotropy}

Despite the inflation in the number of anisotropy indices already proposed, the fractional and relative anisotropy indices are the ones most widely used. Since all these measures are based on the eigenvalue calculation, they cannot be used for higher rank tensors. This motivated Orazlan et al. [46] to define the normalized diffusivity function:

$$
\begin{gathered}
D_{N}(g)=\frac{D(g)}{\operatorname{gentr}(D(g)} \\
\operatorname{gentr}\left(D_{N}(g)^{2}\right)=\frac{1}{3\langle D\rangle^{2}} \sum_{k_{1}=1}^{N_{l}} \sum_{k_{2}=1}^{N_{l}} \mu_{k_{1}} \mu_{k_{2}} D_{k_{1}} D_{k_{2}} \gamma_{k_{1} k_{2}}^{-1}
\end{gathered}
$$


where

$$
\gamma_{k_{1} k_{2}}^{-1}=\frac{1}{2 \pi}\left(\int_{\Omega} d u \prod_{p_{1}=1}^{l} \prod_{p_{2}=1}^{l} U_{k_{1}\left(p_{1}\right)} U_{k_{2}\left(p_{2}\right)}\right)
$$

where $\mu_{k_{1}}=l ! / n_{1 x} ! n_{1 y} ! n_{1 z} !$ is the multiplicity of the $k_{1}^{t h}$ unique element of the DT $D_{k_{1}}$, and $U_{k_{1}\left(p_{1}\right)}$ is the component of the unit vector specified by the $p_{1}^{t h}$ index of the $k_{1}^{t h}$ unique element of the DT. Note that in the expression for $\mu_{k_{1}}, n_{1 x}, n_{1 y}$, and $n_{1 z}$ are respectively the number of $\mathrm{x}, \mathrm{y}$ and $\mathrm{z}$ indices in the full sequence of subscripts defining the component of the tensor. The gamma values defined in Equation (3.10)can be evaluated analytically, and the resulting expressions are listed in Table (3.1) for tensors up to rank-4. In this table, $N_{>}, N_{0}$, and $N_{<}$are respectively the maximum, median, and minimum values of the array $\left(n_{1 x}+n_{2 x}, n_{1 y}+n_{2 y}, n_{1 z}+\right.$ $\left.n_{2 z}\right) \cdot \gamma_{k_{1} k_{2}}^{-1}$ values resulting from other possibilities of $N_{>}, N_{0}$, and $N_{<}$are 0 , and do not contribute to the gentr $\left(D_{N}(u)^{2}\right)$. The variance of the normalized diffusivites is a

Table 3.1 The Gamma Values That Are Needed for the Calculation of the $\operatorname{Gentr}\left(D_{N}(u)^{2}\right)$ Values for Tensor Models up to Rank Six

\begin{tabular}{ccccc}
\hline \hline Rank & $N_{>}$ & $N_{0}$ & $N_{<}$ & $\gamma_{k_{1} k_{2}}$ \\
\hline $\mathrm{l}=0$ & 0 & 0 & 0 & 1 \\
$\mathrm{l}=2$ & 4 & 0 & 0 & 5 \\
& 2 & 2 & 0 & 15 \\
$\mathrm{l}=4$ & 8 & 0 & 0 & 9 \\
& 6 & 2 & 0 & 63 \\
& 4 & 4 & 0 & 105 \\
& 4 & 2 & 2 & 315 \\
\hline
\end{tabular}

measure of anisotropy, and is calculated by:

$$
V=\operatorname{variance}\left(D_{N}(u)\right)=\left\langle D_{N}(u)^{2}-\left\langle D_{N}(u)\right\rangle^{2}\right\rangle=\frac{1}{3}\left(\operatorname{gentr}\left(D_{N}(u)^{2}\right)-\frac{1}{3}\right)
$$


It takes its minimum value of 0 in case of isotropic material, and 1 in case of anisotropic. When a rank-l tensor model is used, this constant diffusivity profile is achieved when all terms except $l=0$ in its irreducible representation (Laplace series) are zero. Independently of the choice of tensor rank, the minimum value for the variance is zero, as expected. Under the condition that all diffusivities implied by a rank-l tensor are nonnegative, the supremum value of the variance is achieved when the tensor is given by a pure outer product of the same 1 vectors, i.e., when the components of the tensor are given by:

$$
D_{i_{1} i_{2} \ldots i_{l}}=D g_{i_{1}}^{\prime} g_{i_{2}}^{\prime} \ldots g_{i_{l}}^{\prime}
$$

where $g^{\prime}$ is the unit vector specifying the direction of greatest diffusion coefficient where D is this maximal diffusivity. A real generalized DT may come arbitrarily close to this, it can never reach this form, since it would imply zero diffusivities along directions perpendicular to $u^{\prime}$. Because the value of zero for diffusivities is nonphysical, the variance associated with the tensor given in Equation (3.12) is referred as the supremum, rather than the maximum value. After performing some algebra, it is possible to show that this supremum value corresponding to a rank-l tensor is given by:

$$
\sup \operatorname{variance}\left(D_{N}(u)\right)=\frac{l^{2}}{9(2 l+1)}
$$

The supremum value depends on the rank of the tensor model selected and that there is an intrinsic limit to the anisotropy that can be quantified with a lower-rank tensor model. From which, the authors defined the generalized anisotropy:

$$
G A=1-\frac{1}{1+(250 V)^{\varepsilon(V)}}
$$

where the exponent $\varepsilon(V)$ is defined as:

$$
\varepsilon(V)=1+\frac{1}{1+5000 V}
$$


The form of the expression for GA as given in Equation (3.14) is not arbitrary, and takes into account the sensitivity of the calculated values to the variations in the variance. Unlike the previously introduced indices, GA also has low contrast among voxels with very low anisotropy values, such as those in free water. As a result, the intensity differences in the GA values are concentrated in voxels within gray matter and the transition from gray matter to white matter, while high intensity is retained in the white matter. If one is interested in changing the contrast according to the values in a different kind of data set, one can easily adjust the constants in the definition of GA [46]. Still, GA is a model based anisotropy measure which is mainly based on the Gaussian model assumption. A new measure will be introduced in chapter 6, which is model free. 


\section{Chapter 4}

\section{Tensor Field Subdivision}

Due to the microscopic scale of the nerve fiber, subdivision of tensors have been introduced as a preprocessing step for DTI data analysis. Different algorithms for interpolation will be first discussed, subdivision in graphics will be then introduced. A novel scheme for tensor field subdivision which is inspired by subdivision surfaces in computer graphics is proposed. The main advantages of this method are its ability to be generalized to Cartesian tensors of all orders and the smoothness imposed on the interpolated field by constraining the divergence and curl of the tensor field. Applying the method involves only a sparse matrix-vector multiplication at each iteration. Results are presented for order 1, 2 and 4 tensors.

\subsection{Introduction}

Voxels in clinical DT images are often quite anisotropic, i.e. not equal in length, width and height. Algorithms tracking white matter tracts can be biased by this anisotropy, and it is therefore recommended to use isotropic voxels. A preliminary resampling step with an adequate interpolation method is therefore important for 
such algorithms. Proper interpolation methods are also required to generalize to the tensor case usual registration techniques used on scalar or vector images.

Several interpolation methodologies were previously used for tensor field interpolation, which is based on the tensor components, tensor invariants, eigenvectors and eigenvalues, Riemannian framework. The algorithms are mainly based on minimizing the invariance of shape or direction or anisotropy between neighbors. These interpolations minimize the number of eigenvectors and eigenvalues computations by restricting it to mesh vertices and makes an exact integration of the tensor lines possible. The tensor field topology is qualitatively the same as for the component wise-interpolation. Since the interpolation decouples the 'shape' and 'direction' interpolation it is shape-preserving, which is especially important for tracing fibers in diffusion MRI data.

\subsubsection{Log Euclidian Interpolation}

This method is based on the fact that a tensor D has a unique symmetric matrix $\operatorname{logarithm} L=\log (S)$. It verifies $S=\exp (L)$ where exp is the matrix exponential. Conversely, each symmetric matrix is associated to a tensor by the exponential. L is obtained from $\mathrm{S}$ by changing its eigenvalues into their natural logarithms, which can be done easily in an orthonormal basis in which $\mathrm{S}$ (and L) is diagonal [54].

Since there is a one-to-one mapping between the tensor space and the vector space of symmetric matrices, one can transfer to tensors the addition + and the scalar multiplication - with the matrix exponential. The logarithmic multiplication is commutative and coincides with matrix multiplication whenever the two tensors $S_{1}$ and $S_{2}$ commute in the matrix sense. With $\odot$ and $\otimes$, the tensor space has, by construction, a vector space structure, which is not the usual structure directly inherited from square matrices. 
When one considers only the multiplication $\odot$ on the tensor space, one has a Lie group structure, i.e. a space which is both a smooth manifold and a group in which algebraic operations are smooth mappings. Among Riemannian metrics in Lie groups, the most convenient in practice, when they exist, are bi-invariant metrics, i.e. distances that are invariant by multiplication and inversion. For the tensor Lie group, bi-invariant metrics exist and are particularly simple, which is named as metrics LogEuclidean metrics [54]:

$$
\operatorname{dist}\left(S_{1}, S_{2}\right)=\left\|\log \left(S_{1}\right)-\log \left(S_{2}\right)\right\|
$$

The log Euclidian algorithm preserves the anisotropy, solves the problem of swelling effect appearing in linear interpolation and preserves the positive definiteness of the tensor [54].

\subsubsection{Geodesic Interpolation}

The interpolation in Riemannian space will have the closed-form expression:

$$
\sigma(t)=\exp _{\sigma_{1}}\left(t \log _{\sigma_{1}}\left(\sigma_{2}\right)\right)+\exp _{\sigma_{2}}\left((1-t) \log _{\sigma_{2}}\left(\sigma_{1}\right)\right)
$$

With the standard matrix coefficient interpolation, the evolution of the trace is perfectly linear, and the principal eigenvalue regularly grows almost linearly, while the smallest eigenvalue slightly grows toward a local maxima before lowering. The disadvantage of this algorithm is that the determinant (i.e., the volume) does not grow regularly in between the two tensors, but goes through a maximum. Moreover, there is a much smoother rotation of the eigenvectors than with the standard interpolation $[55,56]$. The geodesic interpolation interpolates determinants exponentially (i.e.,

the exponents). Thus, it preserves the determinant. The mean of two tensors computed by the standard method will in general be different from the mean computed 

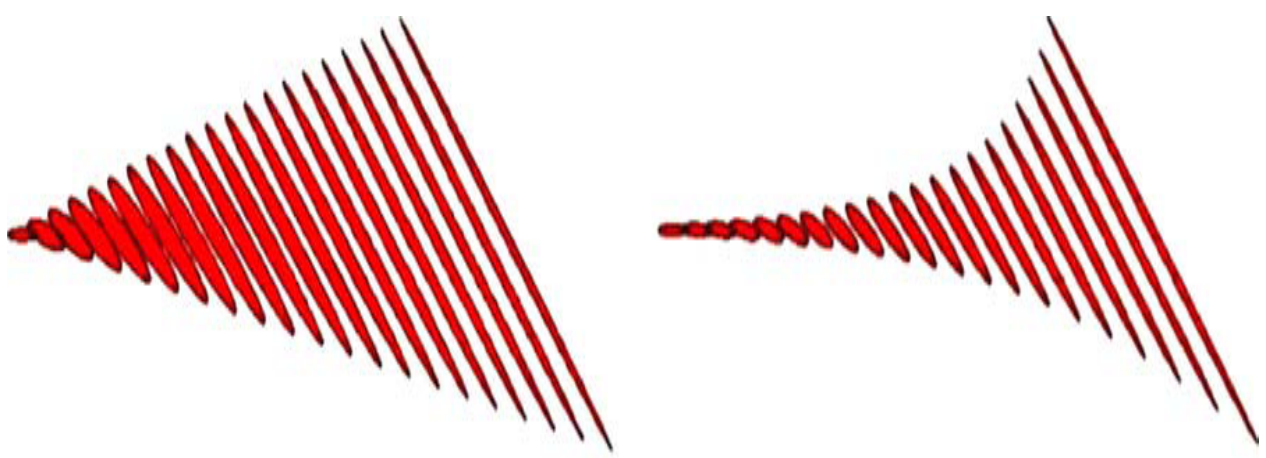

Figure 4.1 Interpolation between 2D tensors of eigenvalues $(5,1)$ horizontally and $(1,50)$ at 45 degrees. Left: Interpolation in the standard matrix space (interpolation of the coefficients). Right: Geodesic interpolation in Riemannian space

using the geodesic distance. When the two tensors are both isotropic but different in size, the standard mean gives the arithmetic mean of the sizes and the geodesic provides the geometric mean. When the two tensors are equal, both means are the same. [57]

\subsubsection{Tensor Spline Interpolation}

Tensor spline interpolation involves the use of a robust tensor product B-spline fitting method involving the minimization of the Riemannian distance between the tensor spline function and the SPD tensor valued data. In order to evaluate a tensor spline, a weighted intrinsic average of SPD tensors is computed [58].

The equation for a $(k-1)^{t h}$ degree B-spline with $(\mathrm{n}+1)$ control points $\left(c_{0}, c_{1},, c_{n}\right)$ and $n+k+1$ numbers called "knots" $\left(t_{-k+1}, t_{-k+2},, t_{n+1}\right)$, is

$$
S(t)=\sum_{i=0}^{n} N_{I, k}(t) c_{i}
$$

Where $t_{0} \leq t \leq t_{n+1-(k-1)}$. Each control point is associated with a basis function 
$N_{i, k}$, where

$$
N_{i, 1}= \begin{cases}0, & t_{i} \leq t<t_{i+1} \\ 1, & \text { otherwise }\end{cases}
$$

and

$$
N_{i, k}(t)=N_{i, k-1}(t) \frac{t-t_{i}}{t_{i+k-1}-t_{i}}+N_{i+1, k-1}(t) \frac{t_{i+k}-t}{t_{i+k}-t_{i+1}}
$$

$N_{i, k}(t)$ functions are polynomials of degree k-1 . Cubic basis functions $N_{I, 4}$ can be used for a third degree B-spline. Knots must be series of monotonically increasing numbers. A more detailed discussion on B-splines can be found in $[58,59]$.

In order to fit a tensor spline to the diffusion tensor data, the control tensors of such a spline are approximated. A tensor spline minimizes the Riemannian distance of the given tensors from the tensor spline curve:

$$
E=\frac{1}{2 N} \sum_{i=0}^{N-1} \operatorname{dist}\left(S\left(t_{i}\right), p_{i}\right)^{2}
$$

In Equation [4.6], the Riemannian metric should be used for the distance calculation, since the tensor space, is a curved manifold (convex cone), a set of control points $\left(c_{0}, c_{1}, \ldots, c_{N-1+k-2}\right)$ that form the spline $S(t)$ which minimizes the energy $E$. The gradient of the square distance between $S\left(t_{i}\right)$ and $P_{i}$ with respect to $S\left(t_{i}\right)$ equals

$$
\nabla_{S\left(t_{i}\right)} \operatorname{dist}\left(S\left(t_{i}\right), p_{i}\right)^{2}=-2 \log _{S\left(t_{i}\right)}\left(P_{i}\right)
$$

where $\log _{S\left(t_{i}\right)}\left(P_{i}\right)$ is the Riemannian logarithmic map, which is a tangent vector at $S\left(t_{i}\right)$. Since the gradient of the energy is with respect to $c_{j}$, the gradient can be expressed in Equation [4.7] by using tangent vectors at point $c_{j}$.

Starting with an initial guess of the control tensors, they are updated by using the gradient descent technique. The new values $c_{j}^{\prime}$ of control tensors will be:

$$
C_{j}^{\prime}=\operatorname{Exp}_{c_{j}}\left(\frac{1}{N} \sum_{i=0}^{N-1} \Lambda_{c_{j}}\left(P_{i}, S\left(t_{i}\right)\right) N_{j, k}\left(t_{i}\right)\right)
$$


Tensor splines can be easily extended to higher dimensional tensor fields. For example, consider the case of a 2-D $(N \times M)$ tensor field. A $(k-1)^{t h}$ degree tensor spline that fits to data requires control tensors and monotonically increasing (in both the dimensions) knots [58]. The tensor spline approximation results in a field which has the noise considerably smoothed out [58].

\subsubsection{Geodesic-Loxodrome Subdivision}

Loxodromes are paths of constant bearing, or paths maintaining a fixed angle with north. let $\mathrm{p}(\phi, \theta)=(r \cos (\theta) \sin (\phi), r \sin (\theta) \sin (\phi), r \cos (\phi))$ be a parametrization of a radius-r globe in $R^{3}$ with $\mathrm{p}(0,0)$ at the north pole. Then $n(x)=-d p / d \phi_{P^{-1}}(x)$ is a tangent to the sphere, pointing north. Let $\hat{\mathrm{n}}(\mathrm{x})=\mathrm{n}(\mathrm{x}) /|\mathrm{n}(\mathrm{x})|$. Then, a loxodrome with unit speed and bearing $\cos ^{-1}(\alpha)$ is traced by a path $\gamma(t)$ on the globe for which:

$$
\left|\gamma^{\prime}(t)\right|=1 \text { and } \gamma^{\prime}(t) \cdot \hat{n}(\gamma(t))=\alpha \text { for all } \mathrm{t}
$$

The path tangent $\gamma^{\prime}(t)$ is also tangent to the sphere, and its constant inner product with $\hat{n}$ implies that $\gamma(t)$ moves northward (or southward) at a constant rate. Geodesicloxodromes similarly move along certain tensor shape parameters at a constant rate, thereby monotonically interpolating tensor shape.

The geodesic-loxodrome is defined as $\gamma(t)$ between $\mathbf{A}$ and $\mathbf{B}$ in $\mathrm{Sym}_{3}$ as the shortest path satisfying:

$$
\gamma(0)=A, \gamma(l)=B,\left|\gamma^{\prime}(t)\right|=1 \text { and } \gamma^{\prime}(t): \hat{\nabla} J_{i}(\gamma(t))=\alpha_{i} \text { for all } \mathrm{t} \epsilon[0, l], i \epsilon 1,2,3
$$

where $\mathrm{l}$ and $\alpha_{i}$ are constants that characterize the path. Geodesic-loxodromes demonstrate the mapping of an intuitive distinction between shape and orientation into a mathematical formulation of interpolation and distance measurement [60]. 
The most important disadvantage of geodesic loxodromes is that it can only apply to $1 \mathrm{D}$ interpolation of 2 nd order tensors.

\subsection{Divergence and Curl Minimizing Subdivision}

The subdivision surfaces, in 3D computer graphics, are defined recursively. The process starts with a given polygonal mesh. A refinement scheme is then applied to this mesh. This process takes that mesh and subdivides it, creating new vertices and new faces. The positions of the new vertices in the mesh are computed based on the positions of nearby old vertices. In some refinement schemes, the positions of old vertices might also be altered (possibly based on the positions of new vertices). This process produces a denser mesh than the original one, containing more polygonal faces. This resulting mesh can be passed through the same refinement scheme again and so on. The limit surface obtained after an infinite number of iterations can be shown to be a smooth surface in some cases - a bicubic B-spline for the scheme of Catmull-Clark [61], and a biquadratic B-spline in the case of Doo-Sabin [62]. The subdivision process is often analyzed as a linear equation $p^{n+1}=S p^{n}$ where $p$ is the set of vertices in the mesh and the superscripts denote iteration number. The subdivision matrix $S$ characterizes the subdivision process of generating new vertices as linear combinations of the old vertices.

Weimer and Warren [63] extended the concept of subdivision to fluid flows. Starting with a coarse vector field representing fluid velocity, their technique generated a dense vector field corresponding to the solution of the Navier-Stokes equation. Similarly, our method can be seen as the solution of a system of partial differential equations. 

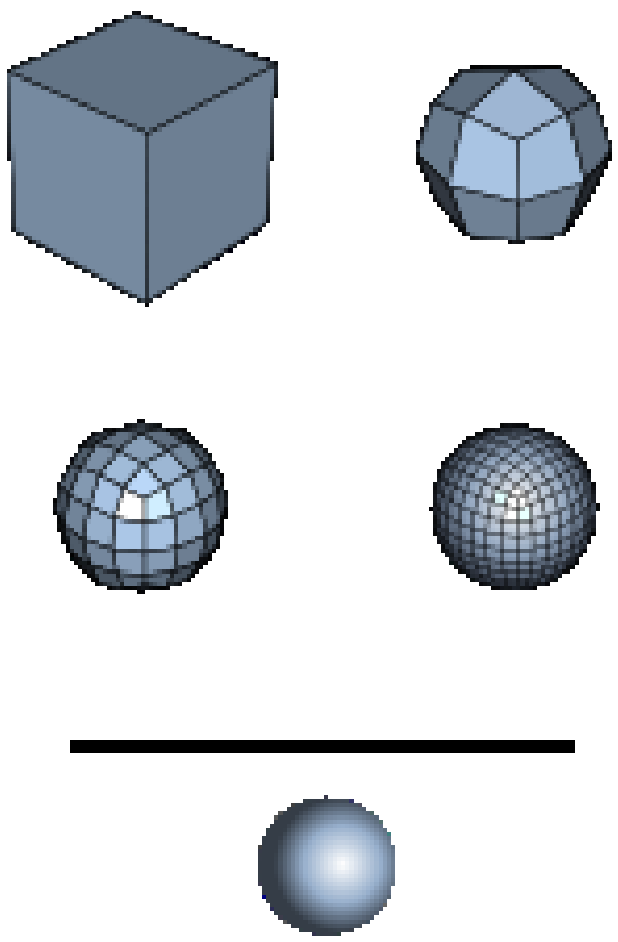

Figure 4.2 First three steps of CatmullClark subdivision of a cube with subdivision surface below 


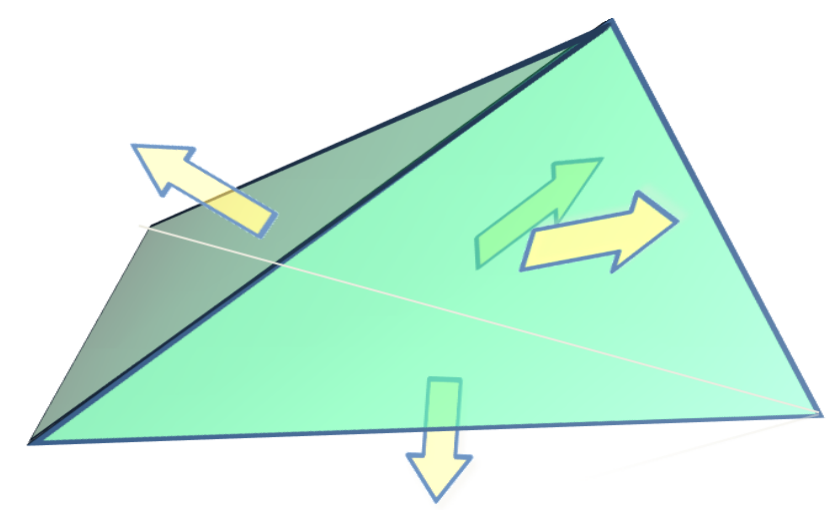

Figure 4.3 Diagram illustrating divergence

\subsubsection{Divergence}

In vector calculus, the divergence is an operator that measures the magnitude of a vector field's source or sink at a given point; the divergence of a vector field is a (signed) scalar. More technically, the divergence represents the volume density of the outward flux of a vector field from an infinitesimal volume around a given point.The inward flux has positive divergence and the outwards flux is negative. The divergence of fluids velocity measures the rate at which fluid is being piped into or out of the region at any point so it is analogous to flux [64].

\subsubsection{Curl}

In fluid dynamics, vorticity is the curl of the fluid velocity. It can also be considered as the circulation per unit area at a point in a fluid flow field. It is a vector quantity, whose direction is along the axis of the fluid's rotation. For a two-dimensional flow, the vorticity vector is perpendicular to the plane.

For a fluid having locally a "rigid rotation" around an axis (i.e., moving like a rotating cylinder), vorticity is twice the angular velocity of a fluid element. An irrotational fluid has no vorticity. Somewhat counter-intuitively, an irrotational fluid can have a non-zero angular velocity (e.g. a fluid rotating around an axis with its tangen- 

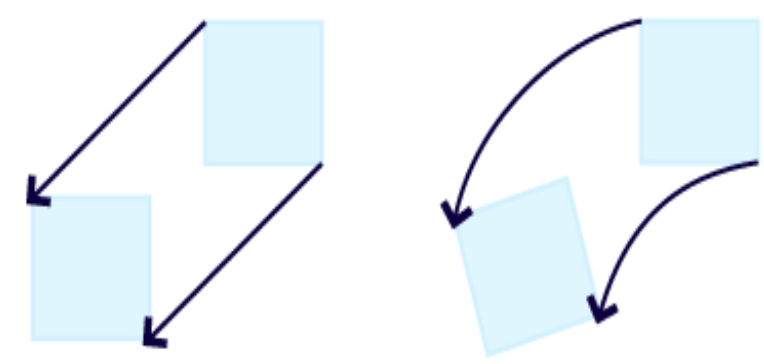

Figure 4.4 Diagram illustrating vorticity in a fluid

tial velocity inversely proportional to the distance to the axis has a zero vorticity).

In general, vorticity is an especially powerful concept in the case that the viscosity is low (i.e. high Reynolds number). In such cases, even when the velocity field is relatively complicated, the vorticity field can be well approximated as zero nearly everywhere except in a small region in space. This is clearly true in the case of 2-D potential flow (i.e. 2-D zero viscosity flow), in which case the flowfield can be identified with the complex plane, and questions about those sorts of flows can be posed as questions in complex analysis which can often be solved (or approximated very well) analytically [65].

\subsubsection{Vector Field Subdivision}

The subdivision scheme for vector field interpolation will be first formulated, which will help explain the tensor field subdivision scheme in the next section of this chapter. the proposed formulation is much simpler than that of Warren and Weimer [63]. Given velocity vectors at the corners of a cube (or square in 2D), a velocity field, which is simultaneously as incompressible and irrotational as possible, is constructed. This can be seen as a physical constraint on the flow, or alternatively since we may wish to interpolate vector fields other than fluid velocity fields, this can also be seen merely 
as a smoothness constraint since spurious sources/sinks and vortices can introduce regions of rapidly changing vector direction and length.

The strength of sources or sinks in a fluid flow can be quantified by the divergence of the velocity field, and the strength of vortices can be quantified by the curl.

$$
\operatorname{div} v=\frac{\partial v_{x}}{\partial x}+\frac{\partial v_{y}}{\partial y}+\frac{\partial v_{z}}{\partial z}, \operatorname{curl} v=\left[\begin{array}{c}
\frac{\partial v_{z}}{\partial y}-\frac{\partial v_{y}}{\partial z} \\
\frac{\partial v_{x}}{\partial z}-\frac{\partial v_{z}}{\partial x} \\
\frac{\partial v_{y}}{\partial x}-\frac{\partial v_{x}}{\partial y}
\end{array}\right]
$$

where $v=\left[v_{x}, v_{y}, v_{z}\right]^{T}$ is the vector field. These are usually denoted by the shorthand $\nabla \cdot v$ and $\nabla \times v$ respectively. We will approximate these operators discretely by using finite differences

$$
\begin{aligned}
\Delta_{x} & =\frac{1}{2}(v(x+1, y, z)-v(x-1, y, z)), \\
\Delta_{x}^{+} & =v(x+1, y, z)-v(x, y, z) \\
\Delta_{x}^{-} & =v(x, y, z)-v(x-1, y, z)
\end{aligned}
$$

which are the central, forward and backward differences respectively. The subdivision operation takes as input a coarse grid of vectors $(2 \times 2$ in $2 \mathrm{D}$, or $2 \times 2 \times 2$ in $3 \mathrm{D})$ we will call $v^{0}$ and produces a refined grid $(3 \times 3$ in $2 \mathrm{D}$, or $3 \times 3 \times 3$ in $3 \mathrm{D})$ we will call $v^{1}$ as shown in Figure (4.5). The process will proceed iteratively and each step will interpolate the results of the previous step. The system of equations which determine $v^{n+1}$ given $v^{n}$ specify 3 types of requirements:

1. Interpolation, the vectors at iteration $n$ should be interpolated in step $n+1$. In the first step we have

$$
\begin{aligned}
& v^{n}(1,1)=v^{n+1}(1,1), \\
& v^{n}(1,3)=v^{n+1}(1,3), \\
& v^{n}(3,1)=v^{n+1}(3,1),
\end{aligned}
$$



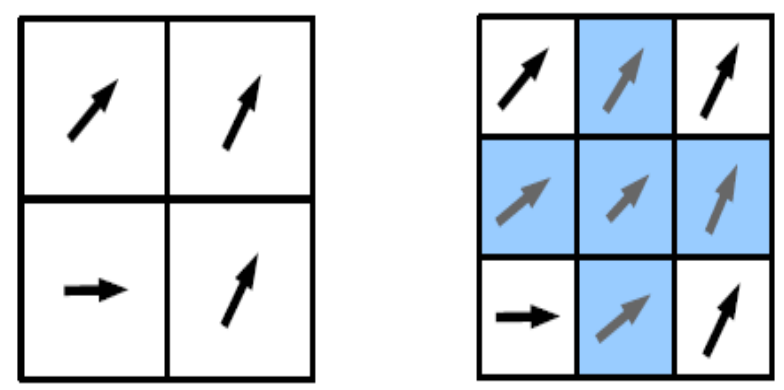

Figure 4.5 Illustration of the subdivision process in 2D. The first subdivision iteration replaces the $2 \times 2$ grid of vectors $\left(v^{0}\right)$ with a $3 \times 3$ grid of vectors $\left(v^{1}\right)$. The vectors in the corners of the domain (white background) are interpolated. The remaining 5 vectors are computed by minimizing the divergence and curl of the field. The next subdivision step would interpolate all 9 vectors. The process can be repeated to obtain $v^{n}$, a grid of size $2^{n}+1 \times 2^{n}+1$

$$
v^{n}(3,3)=v^{n+1}(3,3)
$$

where the array $v^{n}$ has been padded to be the same size as $v^{n+1}$ so that indices at corresponding corners are equal.

2. Divergence minimization, the divergence at each point in $v^{n+1}$ is set to zero, and written in terms of $v^{n}$ when a corner point is involved. If the central difference equation involves a point outside the domain, forward or backward differences are used instead. There will be one equation for each vector in $v^{n+1}$. Each equation will be of the form

$$
0=\Delta_{x} v_{x}+\Delta_{y} v_{y}+\Delta_{z} v_{z}
$$

The superscript on $v$ is $n+1$ for the new voxels being computed, and $n$ for the voxels being interpolated.

3. Curl minimization, the curl is handled analogously to the divergence. For the $2 \mathrm{D}$ example there is only one nonzero component of the curl for each vector. In 


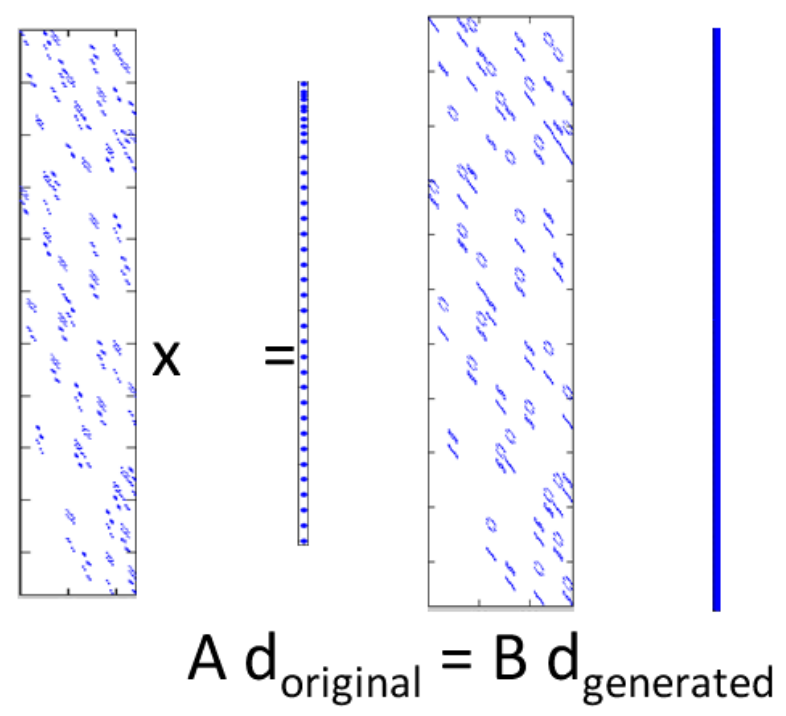

Figure 4.6 Sparse A and B matrices.

the 3D case there will be 3 components per voxel of the form

$$
\begin{aligned}
& 0=\Delta_{y} v_{z}-\Delta_{z} v_{y}, \\
& 0=\Delta_{z} v_{x}-\Delta_{x} v_{z}, \\
& 0=\Delta_{x} v_{y}-\Delta_{y} v_{x}
\end{aligned}
$$

for a total of 81 equations in the first step.

By reshaping $v$ into column vector the equations can be rearranged in the form

$$
0=A v^{n}+B v^{n+1}
$$

Both matrices $A$ and $B$ are sparse, as shown in figure (4.6), and contain only elements with values $\left(-1,-\frac{1}{2}, 0, \frac{1}{2}, 1\right)$. Overall, in the $2 \mathrm{D}$ case we have to solve for 18 vector components in $v^{n+1}$ given 22 equations. In 3D we solve for 81 vector components given 112 equations. The equations are solved in the least squares sense by:

$$
v^{n+1}=-B^{+} A v^{n}
$$



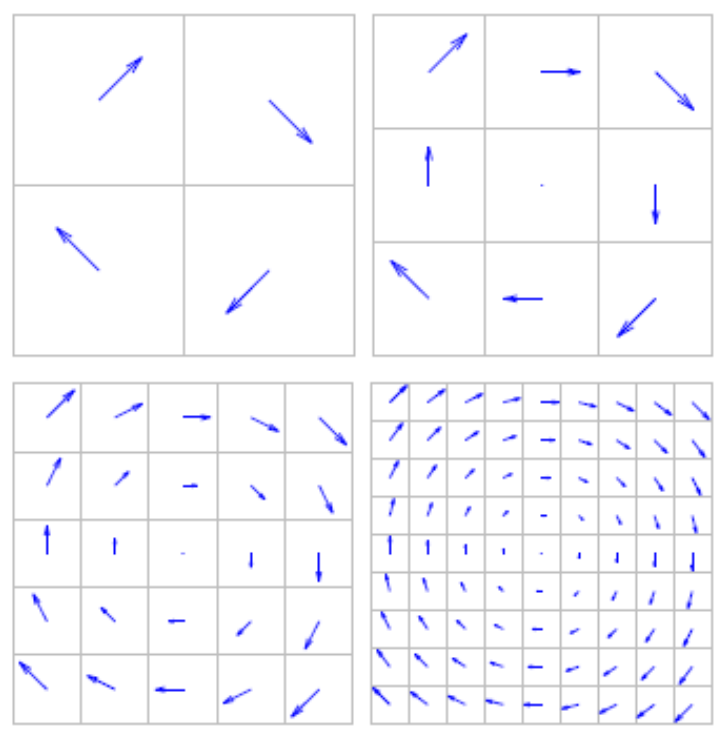

Figure 4.7 Vector Field Subdivision of rotational vector. The field to be interpolated (top left) is subdivided 3 times (results shown top to down and left to right).

where the pseudoinverse $B^{+}=\left(B^{T} B\right)^{-1} B^{T}$. This is a subdivision scheme in which the subdivision matrix is $S=-B^{+} A$. The result is a vector field where the magnitudes of the divergence and curl are minimized while interpolating the coarse vector field. The influence of the divergence and curl minimization can be separately controlled by using a weighted least squares approach. We implement this by scaling the divergence equations in Equation (4.16) by $\sigma_{d i v}=0.9$ and the curl equations by $\sigma_{c u r l}=0.1$.

Results of vector field interpolation are shown in Figures (4.7, 4.8, 4.9, 4.10). Note that even though curl and divergence are minimized in the least squares sense they are not guaranteed to equal zero. The subdivision process can generate rotational and nonsolenoidal flows.

\subsubsection{Tensor Field Subdivision}

The vector field interpolation results of the previous section will be extended to tensor fields. The same constraints (interpolation, divergence minimization and curl 

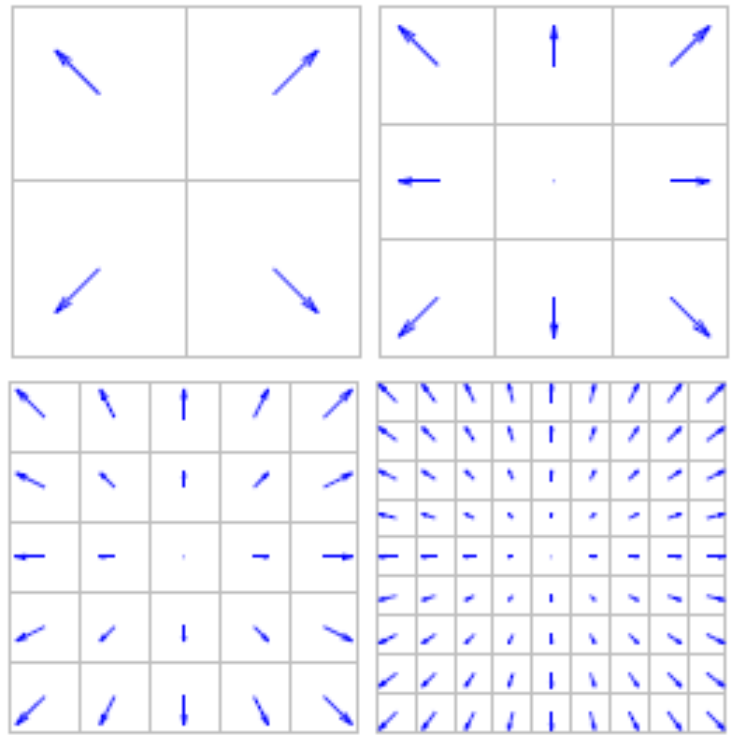

Figure 4.8 Vector Field Subdivision of nonsolenoidal vector. The field to be interpolated (top left) is subdivided 3 times (results shown top to down and left to right).

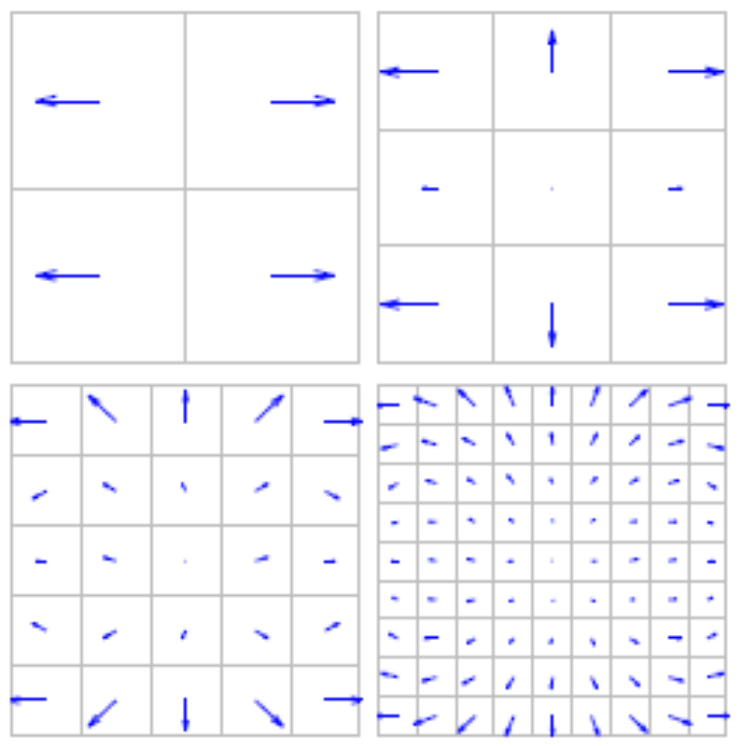

Figure 4.9 Vector Field Subdivision of rotational vector. The field to be interpolated (top left) is subdivided 3 times (results shown top to down and left to right). 


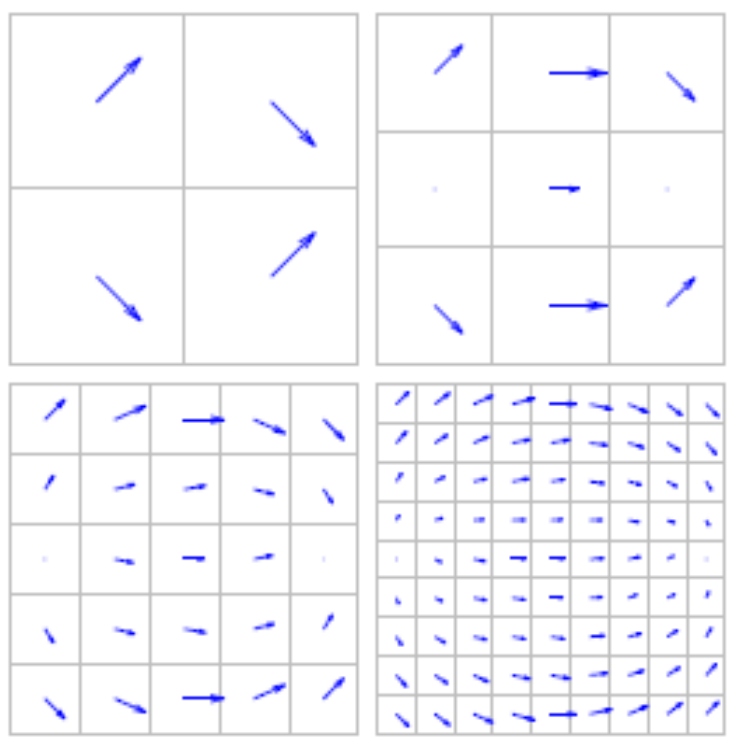

Figure 4.10 Vector Field Subdivision of nonsolenoidal vector. The field to be interpolated (top left) is subdivided 3 times (results shown top to down and left to right).

minimization) were used by simply substituting the definitions of the divergence and curl of tensors of arbitrary order.

\subsubsection{Rank-2 Tensor Subdivision}

The divergence of a rank-2 tensor field is a vector field of the same dimension. For a symmetric tensor we have

$$
\begin{gathered}
\operatorname{div}\left[\begin{array}{cc}
D_{x x} & D_{x y} \\
D_{x y} & D_{y y}
\end{array}\right]=\left[\begin{array}{c}
\frac{\partial D_{x x}}{\partial x}+\frac{\partial D_{x y}}{\partial y} \\
\frac{\partial D_{x y}}{\partial x}+\frac{\partial D_{y y}}{\partial y}
\end{array}\right] \\
\operatorname{div}\left[\begin{array}{ccc}
D_{x x} & D_{x y} & D_{x z} \\
D_{x y} & D_{y y} & D_{y z} \\
D_{x z} & D_{y z} & D_{z z}
\end{array}\right]=\left[\begin{array}{c}
\frac{\partial D_{x x}}{\partial x}+\frac{\partial D_{x y}}{\partial y}+\frac{\partial D_{x z}}{\partial z} \\
\frac{\partial D_{x y}}{\partial x}+\frac{\partial D_{y y}}{\partial y}+\frac{\partial D_{y z}}{\partial z} \\
\frac{\partial D_{x z}}{\partial x}+\frac{\partial D_{y z}}{\partial y}+\frac{\partial D_{z z}}{\partial z}
\end{array}\right] .
\end{gathered}
$$

To perform interpolation we form an equation for each of the vector components in Equation (4.18) or (4.19). For each such equation the corresponding row of matrices $A, B$ has the appropriate elements assigned. 
A good intuition can be gained about the nature of vector divergence by observing that near sources the vector field has positive divergence and locally the vectors appear to point away from the source. Conversely, near a sink the vector appear to converge toward the sink. The meaning of tensor field divergence can be appreciated by considering the diffusion equation when the concentration gradient is constant, but not necessarily zero

$$
\frac{\partial C}{\partial t}=\operatorname{div}(D \nabla C)=\operatorname{div}(D) \cdot \nabla C
$$

Then at steady state $\frac{\partial C}{\partial t}=0$ is achieved for $\operatorname{div}(D)=0$. Under the given conditions, this is equivalent to saying that the inhomogeneous tensor field $D$ does not transform any constant vector field into a vector field with nonzero divergence.

In general, the divergence of a tensor field of rank $n$ is a tensor field of orderrank $(n-1)$ given in Einstein notation as $\partial_{i} D_{i}$. This notation indicates that for all possible values of index $i$, the tensor components are differentiated with respect to that index and summed over. Note that when the field consists of totally symmetric tensors the divergence tensor is also totally symmetric.

The curl of a rank 2 tensor field is a vector in $2 \mathrm{D}$ and $3 \mathrm{D}$,

$$
\begin{gathered}
\operatorname{curl}\left[\begin{array}{cc}
D_{x x} & D_{x y} \\
D_{x y} & D_{y y}
\end{array}\right]=\left[\begin{array}{c}
\frac{\partial D_{x y}}{\partial x}-\frac{\partial D_{x x}}{\partial y} \\
\frac{\partial D_{y y}}{\partial x}-\frac{\partial D_{x y}}{\partial y}
\end{array}\right] \\
\operatorname{curl}\left[\begin{array}{ccc}
D_{x x} & D_{x y} & D_{x z} \\
D_{x y} & D_{y y} & D_{y z} \\
D_{x z} & D_{y z} & D_{z z}
\end{array}\right]=\left[\begin{array}{ccc}
\frac{\partial D_{x z}}{\partial y}-\frac{\partial D_{x y}}{\partial z} & \frac{\partial D_{y z}}{\partial y}-\frac{\partial D_{y y}}{\partial z} & \frac{\partial D_{z z}}{\partial y}-\frac{\partial D_{y z}}{\partial z} \\
\frac{\partial D_{x x}}{\partial z}-\frac{\partial D_{x z}}{\partial x} & \frac{\partial D_{x y}}{\partial z}-\frac{\partial D_{y z}}{\partial x} & \frac{\partial D_{x z}}{\partial z}-\frac{\partial D_{z z}}{\partial x} \\
\frac{\partial D_{x y}}{\partial x}-\frac{\partial D_{x x}}{\partial y} & \frac{\partial D_{y y}}{\partial x}-\frac{\partial D_{x y}}{\partial y} & \frac{\partial D_{y z}}{\partial x}-\frac{\partial D_{x z}}{\partial y}
\end{array}\right] .
\end{gathered}
$$

The curl of a tensor field of rank $n$ is a tensor field of rank $(n+d-3)$ in $d$ dimensions 
defined as $\varepsilon_{i j k}\left(\partial_{j} D_{k}\right)$ where $\varepsilon_{i j k}$ is the Levi-Civita symbol (permutation tensor)

$$
\varepsilon_{i j k}= \begin{cases}+1 & (i, j, k) \text { is an even permutation of indices } \\ -1 & (i, j, k) \text { is an odd permutation of indices } \\ 0 & \text { otherwise }\end{cases}
$$

\subsubsection{Rank-4 Tensor Subdivision}

The divergence and curl can then be generalized for the rank 4 tensor, and even any higher order tensor with increasing the number of equations. In case of 3D rank-4 tensor, the number of unknowns will be $(15 \times 19)$.we will be having 8 equations for interpolation, 270 equations from divergence and 810 equations from curl, which can be solved using least squares.

\subsubsection{Bézier Curves and Splines}

A Bézier curve is a parametric curve important in computer graphics and related fields. Generalizations of Bézier curves to higher dimensions are called Bézier surfaces, of which the Bézier triangle is a special case. In vector graphics, Bézier curves are an important tool used to model smooth curves that can be scaled indefinitely. "Paths," as they are commonly referred to in image manipulation programs [66]. The presented spline will take the form of a tensor-valued Bézier curve. The Bernstein polynomials [67] which form the basis of the Bézier curve of degree $\mathrm{n}$ are given by

$$
B_{i}^{n}(t)=\left(\begin{array}{c}
n \\
i
\end{array}\right) t^{i}(1-t)^{n-i}
$$

where the binomial coefficients are given by

$$
\left(\begin{array}{c}
n \\
i
\end{array}\right)=\left\{\begin{array}{cc}
\frac{n !}{i !(n-i) !} & \text { if } 0 \leq i \leq n \\
0 & \text { Otherwise }
\end{array}\right.
$$


A tensor-valued 3d volume can be defined in terms of the basis functions 4 as

$$
D(u, v, w)=\sum_{i=0}^{n} \sum_{j=0}^{n} \sum_{k=0}^{n} D^{(i, j, k)} B_{i}^{n}(u) B_{j}^{n}(v) B_{k}^{n}(w)
$$

where $D^{(i, j, k)}$ are the control tensors. The Bernstein form of the Bézier curve permits the derivatives of a patch to be computed by simply computing the differences of control points. For example, the derivative in the u-direction of the patch 6 is given by

$$
\partial_{u} D(u, v, w)=\sum_{i=0}^{n-1} \sum_{j=0}^{n} \sum_{k=0}^{n}\left(D^{(i+1, j, k)}-D^{(i, j, k)}\right) B_{i}^{n-1}(u) B_{j}^{n}(v) B_{k}^{n}(w)
$$

Continuity across the boundary between adjacent patches C,D can be obtained by imposing the constraint $C^{(n)}=B^{(0)}$. Smoothness across the boundary between adjacent patches $\mathrm{C}$, D can be obtained by imposing the constraint

$$
C^{n}-C^{n-1}=\alpha\left(D^{(1)}-D^{(0)}\right)
$$

with $\alpha>0$ for $C^{1}$ continuity and $\alpha=1$ for $G^{1}$ continuity. Note that the derivative operation reduces the degree of the patch by one in the direction being differentiated. We can add and subtract patches by simply adding and subtracting control points as long as the patches being operated on have the same degree in each direction. In computing the divergence and curl of patches we will utilize the degree elevation operation on patches to achieve this condition. for a degree $n+1$ curve with control points $\mathrm{E}$, the smoothness function can be calculated using the following function

$$
E^{(i)}=\frac{i}{n+1} D^{(i-1)}+\left(1-\frac{i}{n+1}\right) D^{(i)}
$$

The differentiation and degree elevation operators for the rank 4 tensor valued spline can be implemented as the matrix vector multiplication $c=D_{x} d$ where the control points have been reshaped into $15(n+1)^{3} 1$ column vectors c, d and $D_{x}$ is a sparse $15(n+1)^{3} 15(n+1)^{3}$ matrix. In an analogous fashion, we can define matrices $D_{y}$ and 
$D_{z}$ which can be used to compute the derivative of the spline in the $y$ and $z$ directions. The rows of these matrices can then be used to create matrices which implement the curl and divergence operators.

\subsubsection{Minimization}

The control points for the tensor-valued patch were computed by solving a system of equations in the least squares sense. The system contains 4 types of equations:

- Interpolation, for interpolation within a cell, we impose a hard constraint that the 8 tensors at the corners of the cell are known.

- Boundary conditions, the derivative across the boundary can be controlled to match derivatives with neighboring voxels, or clamp the derivative at the boundary of the dataset.

- Divergence minimization. The divergence of the spline can be expressed in terms of the control vertices. Minimization of the divergence can be seen as a physical constraint which favors conservation of mass.

- Curl minimization, the curl of the spline can also be expressed in terms of the control vertices. These constraints impose additional smoothness on the resulting tensor field.

The boundary, divergence and curl constraints have associated weights $\left(\sigma_{b c}, \sigma_{d i v}, \sigma_{c u r l}\right)$ which permit controlling the influence of each type of constraint independently.

The input to the Algorithm will be the corners of the slice, if it is $2 \mathrm{D}$ subdivision or the 4 corners of the volume in case of the 3D volume subdivision as shown in Figures $(4.11,4.12)$ respectively. The divergence and curl functions will be calculated for 

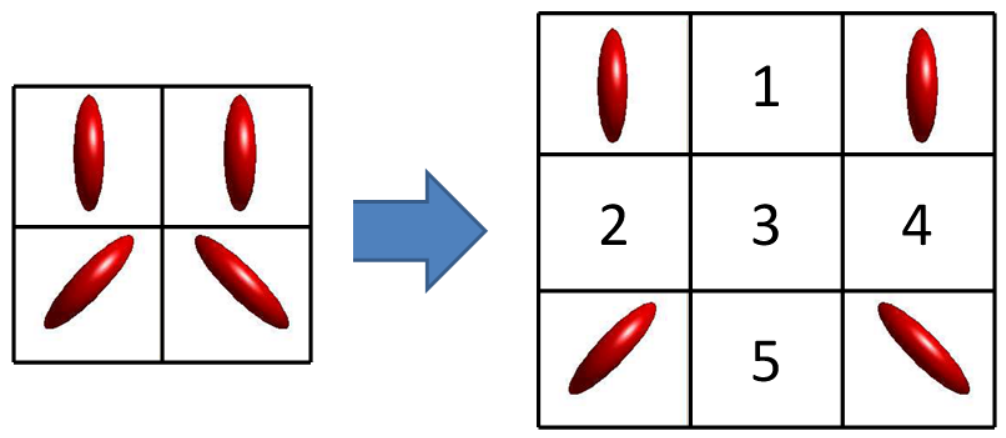

Figure 4.11 Input and Output voxels in case of 2D subdivision
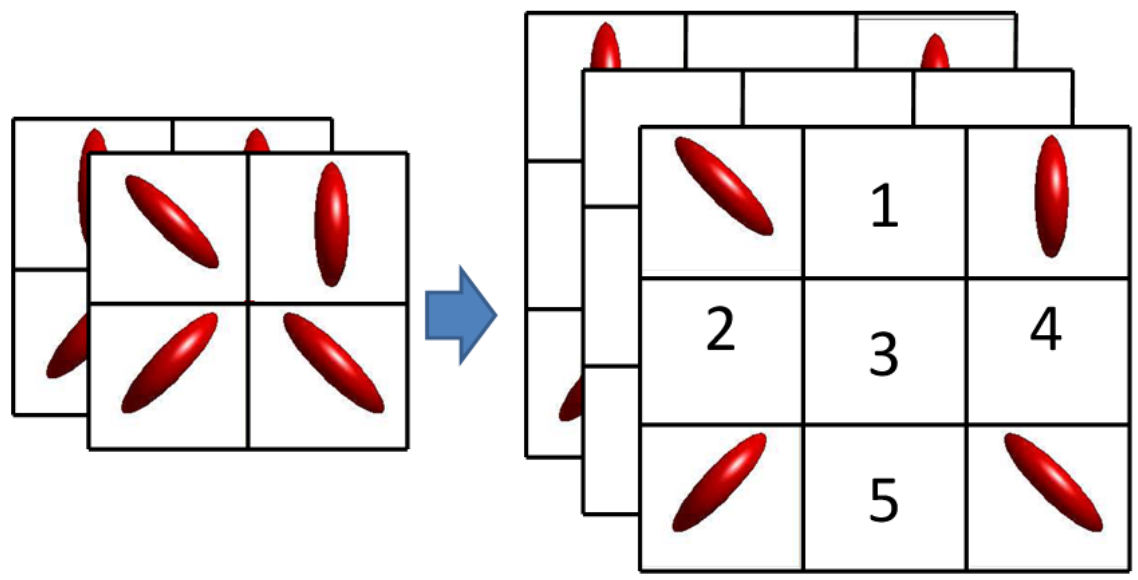

Figure 4.12 Input and Output voxels in case of 3D subdivision 


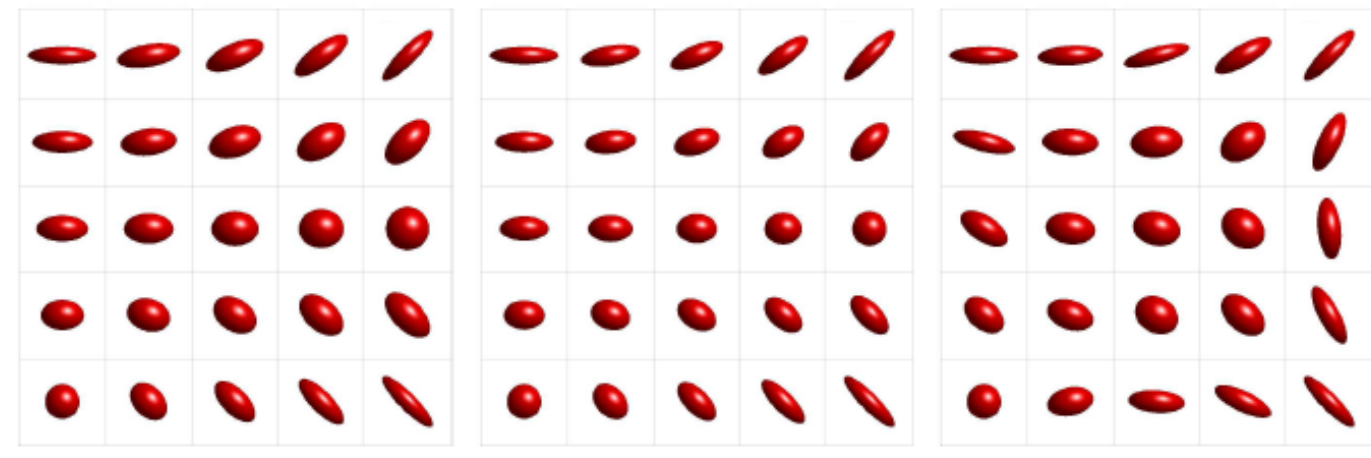

Figure 4.13 Rank 2 tensor field interpolation. Linear interpolation (left), Log-Euclidean interpolation (center), 2 subdivision steps (right).

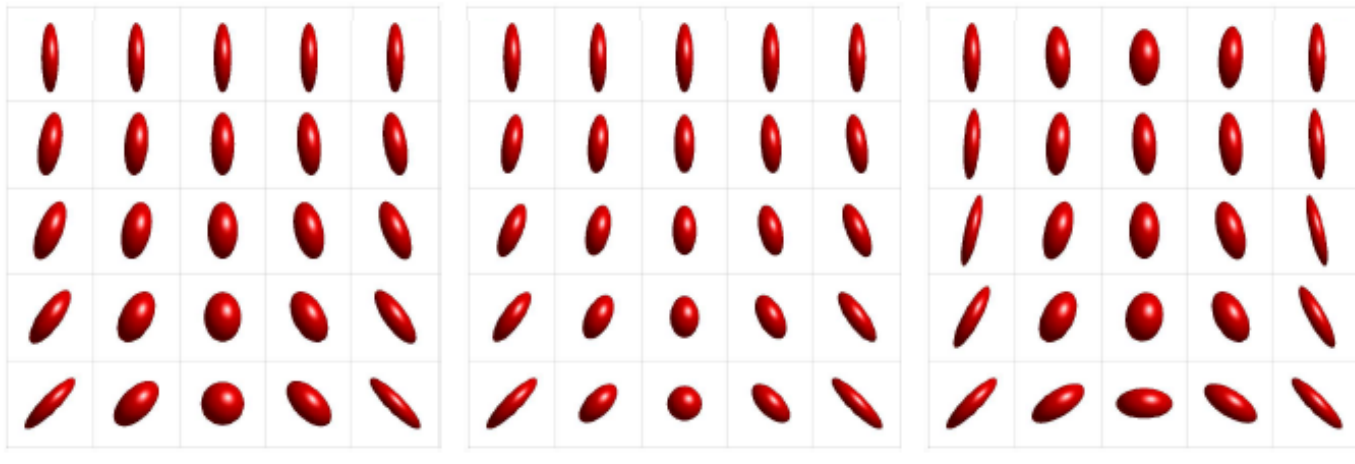

Figure 4.14 Rank 2 tensor field interpolation. Linear interpolation (left), Log-Euclidean interpolation (center), 2 subdivision steps (right).

each voxel, and using least square, the generated voxels can be calculated

$$
A d_{o}=B d_{N} d_{N}=\left(B^{T} B\right)^{-1} B^{T} A d_{o}
$$

Both matrices $\mathrm{A}$ and $\mathrm{B}$ are sparse. The equation is then solved for $\mathrm{d}$ in the least squares sense using sparse Cholesky factorization.

\subsubsection{Subdivision Results and Discussion}

The results of rank 2 tensor field subdivision are shown in Figures $(4.13,4.14)$, along with linear and log-Euclidean interpolation for comparison. Note that in the bottom row of voxels in both examples (top and bottom of Figure $(4.13,4.14)$ ) FA is better 
preserved for the subdivision scheme than in the linear and log-Euclidean interpolation cases. The subdivision scheme results in a smooth rotation of the diffusion tensor.

To measure the difference in estimation between the linear and the subdivision algorithms, several $(9 \times 9)$ synthetic rank-2 tensor slices were generated, to form the isotropic slice, a diagonal fiber with random direction, 2 diagonal crossing fibers with random directions, 2 kissing fibers, bifurcating fibers, solenoid source with random position, exponential decay, a bifurcating fiber as shown in figure. Odd index voxels, forming a $(4 \times 4)$ slices, were used as input for both linearinterpolation and subdivision algorithms. The average Frobenius norm error between the output tensor to the corresponding tensor of the ground truth slice is calculated, to measure of the error between the estimated tensor and the original one. The highest error occurred in case of Solenoidal slice. Figures $(4.15,4.16,4.17)$ are examples output of subdivision and linear interpolation algorithms. the anisotropy is more preserved in case of the subdivision than the interpolation. In case of the solenoidal slice in Figure [4.15], this effect can be seen at the top and bottom row of the output slices. For the kissing fibers in Figure [4.16],It can be seen in the left column and the bottom row.

These different synthetic data were randomly used for subdivision and interpolation, the mean, minimum and maximum average Frobenius norm was in both cases as shown in Table (4.1).

Results for rank 2 Bézier spline tensor interpolation of synthetic data are shown in Figures $(4.18,4.19,4.20,4.21)$. The polynomial degree, n, was varied from 1 to 7 . For $\mathrm{n}=1$ the Bézier patch we compute reduces to linear interpolation. Results are also compared with the subdivision approach in [68]. The background color indicates fractional anisotropy (FA). Note the behavior in the bottom row of voxels in Figures (4.18, 4.22) as the degree of the interpolating curve increases the better that anisotropy is 


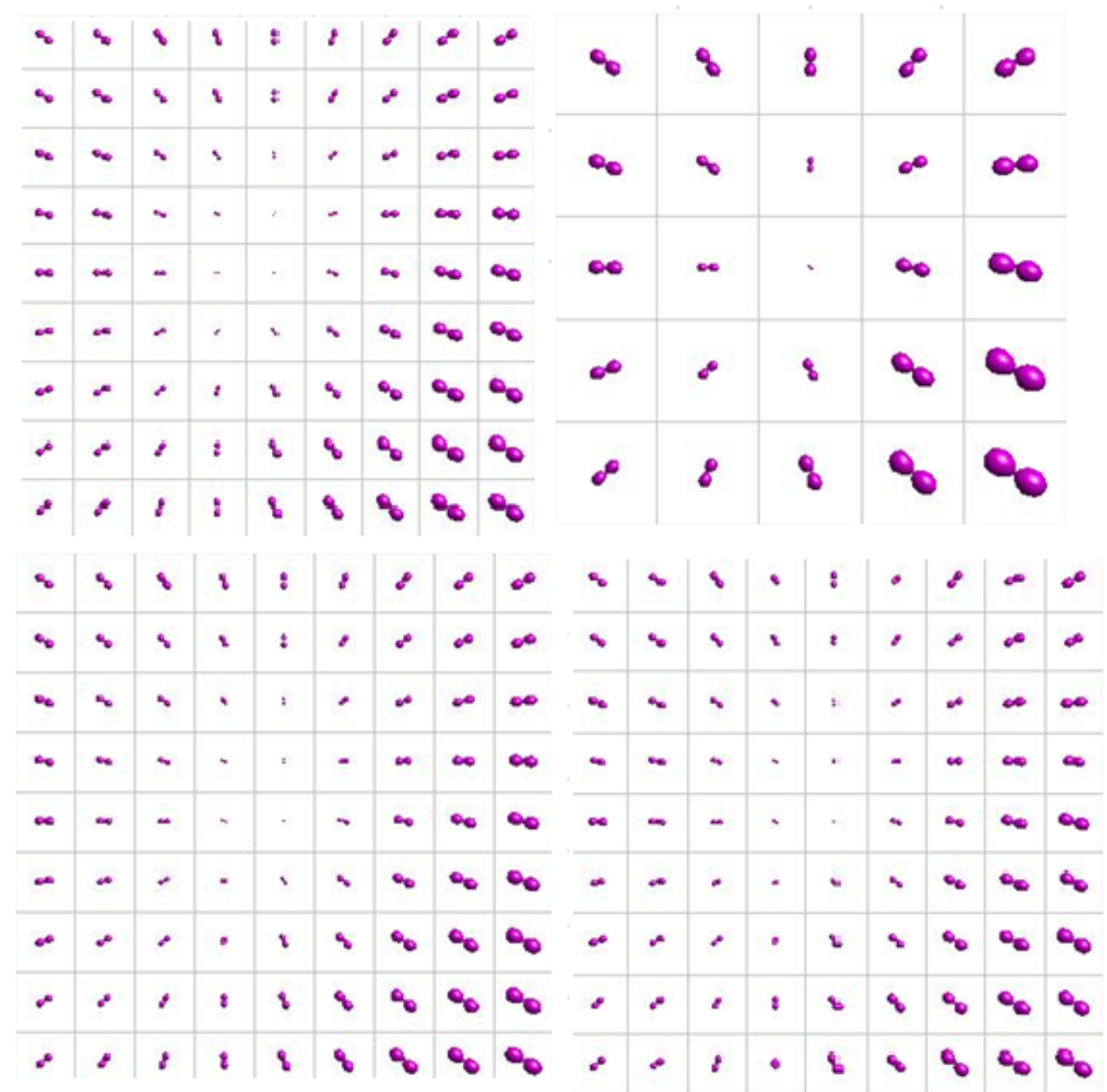

Figure 4.15 Solenoidal, Rank 2 tensor, Top: (Right) Ground truth slice, (Left) Input slice, Bottom: (Right) Subdivision Output, (Left)Linear Interpolation Output. 

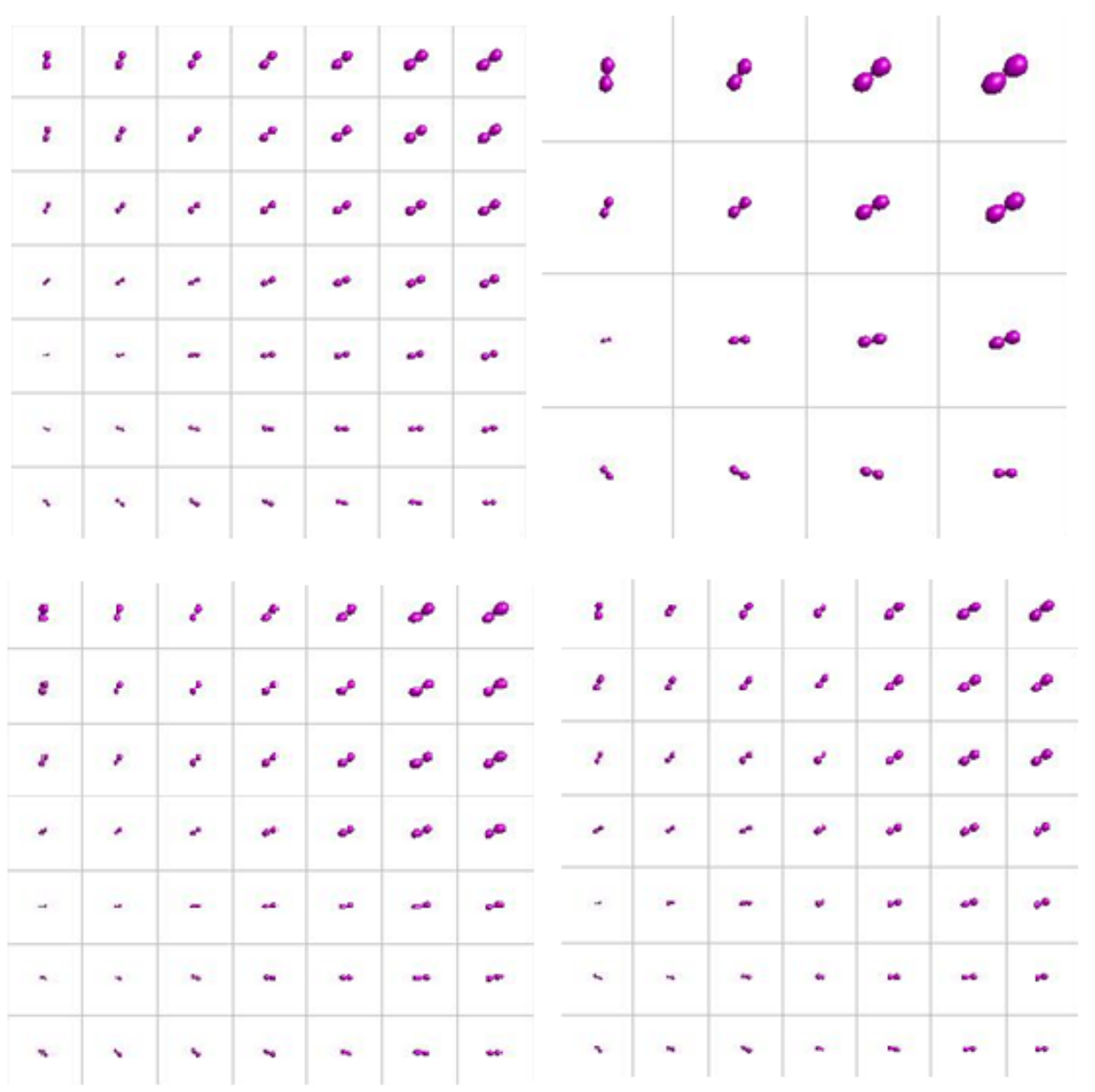

Figure 4.16 Exponential decay, Rank 2 tensor, Top: (Right) Ground truth slice, (Left) Input slice, Bottom: (Right) Subdivision Output, (Left)Linear Interpolation Output. 


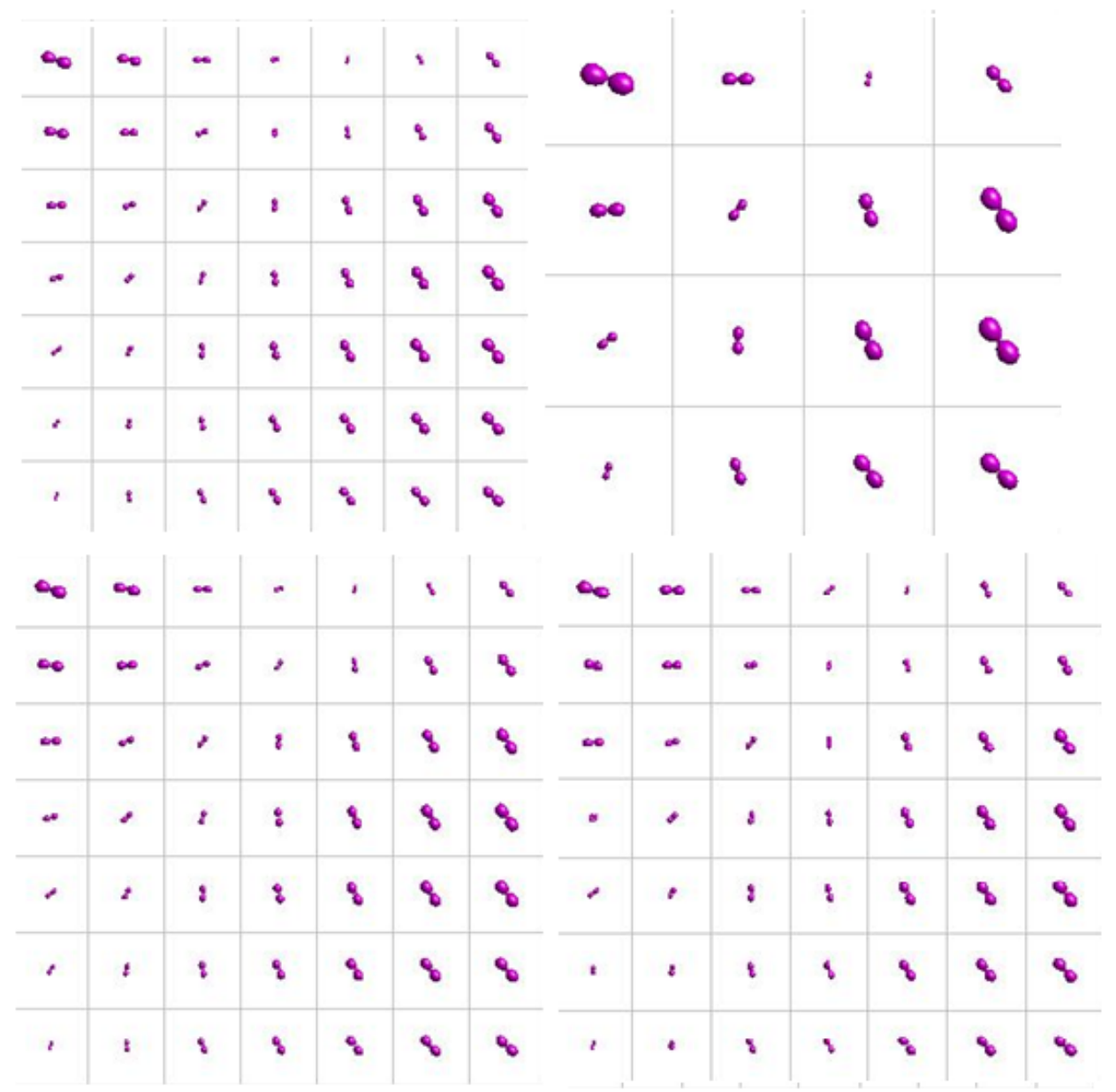

Figure 4.17 kissing fibers, Rank 2 tensor, Top: (Right) Ground truth slice, (Left) Input slice, Bottom: (Right) Subdivision Output, (Left)Linear Interpolation Output. 

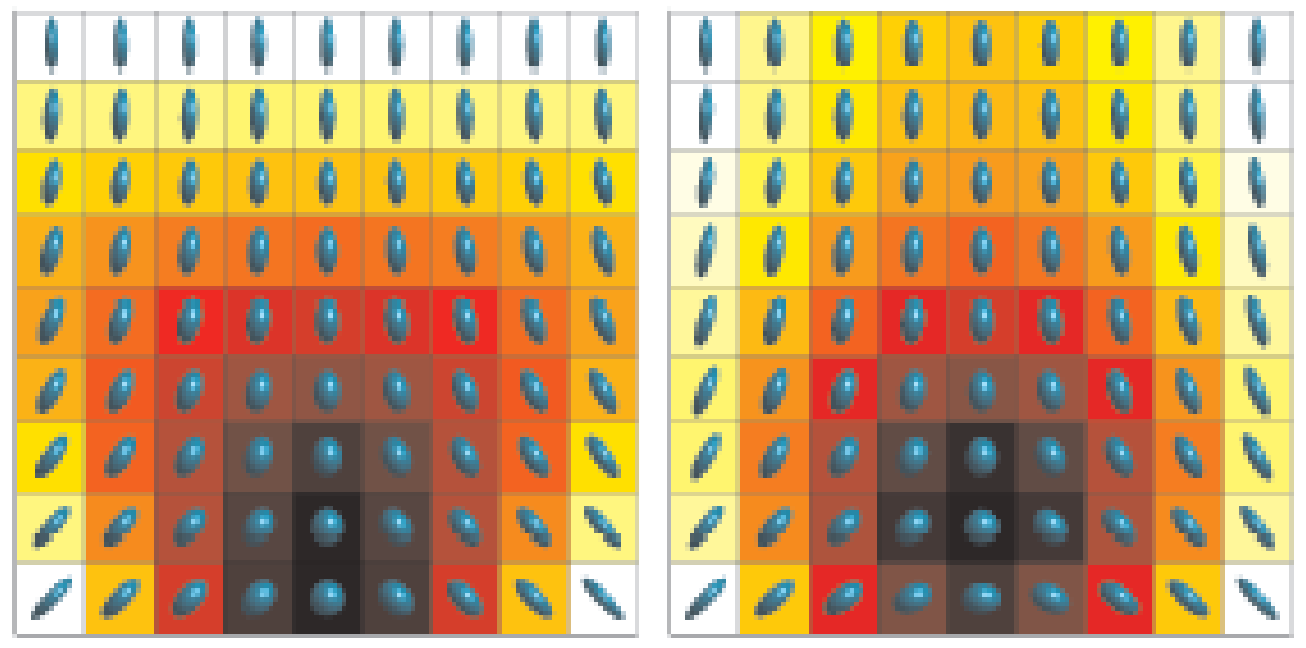

Figure 4.18 Bézier spline Interpolation, Rank 2 tensor results with Degree $\mathrm{n}=1$ (left) and $\mathrm{n}=2$ (right).
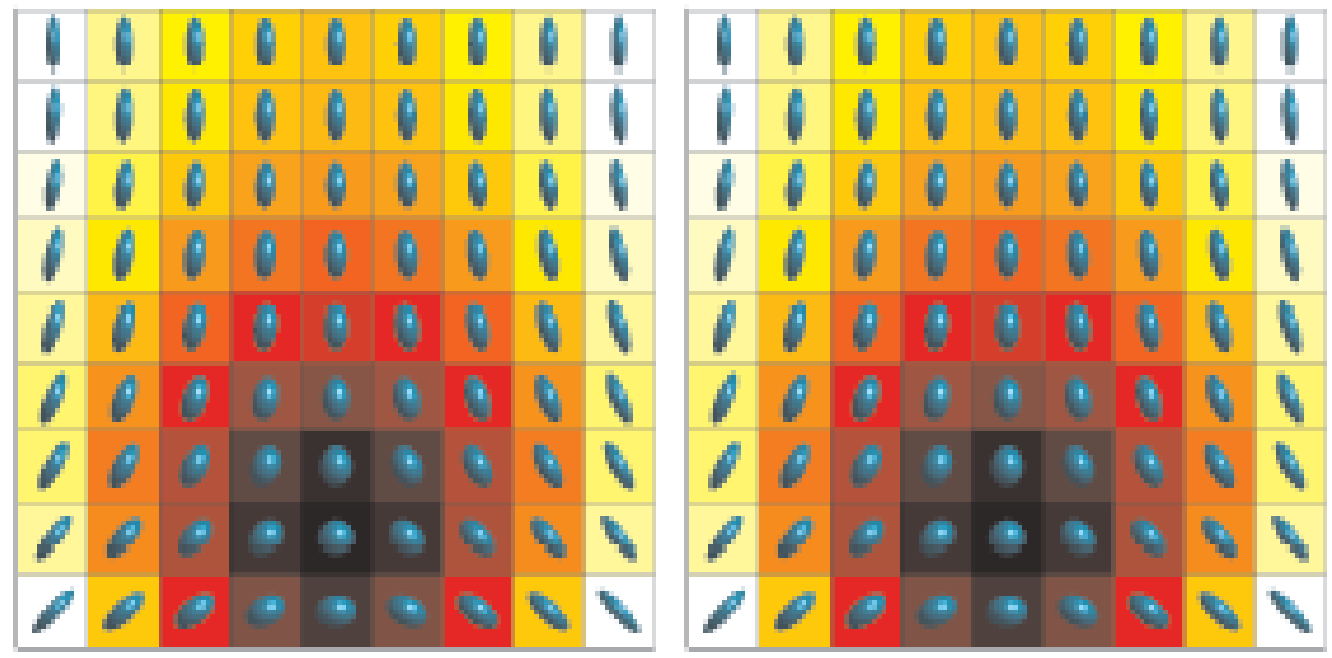

Figure 4.19 Bézier spline Interpolation, Rank 2 tensor results with Degree $\mathrm{n}=3$ (left) and $\mathrm{n}=4$ (right). 

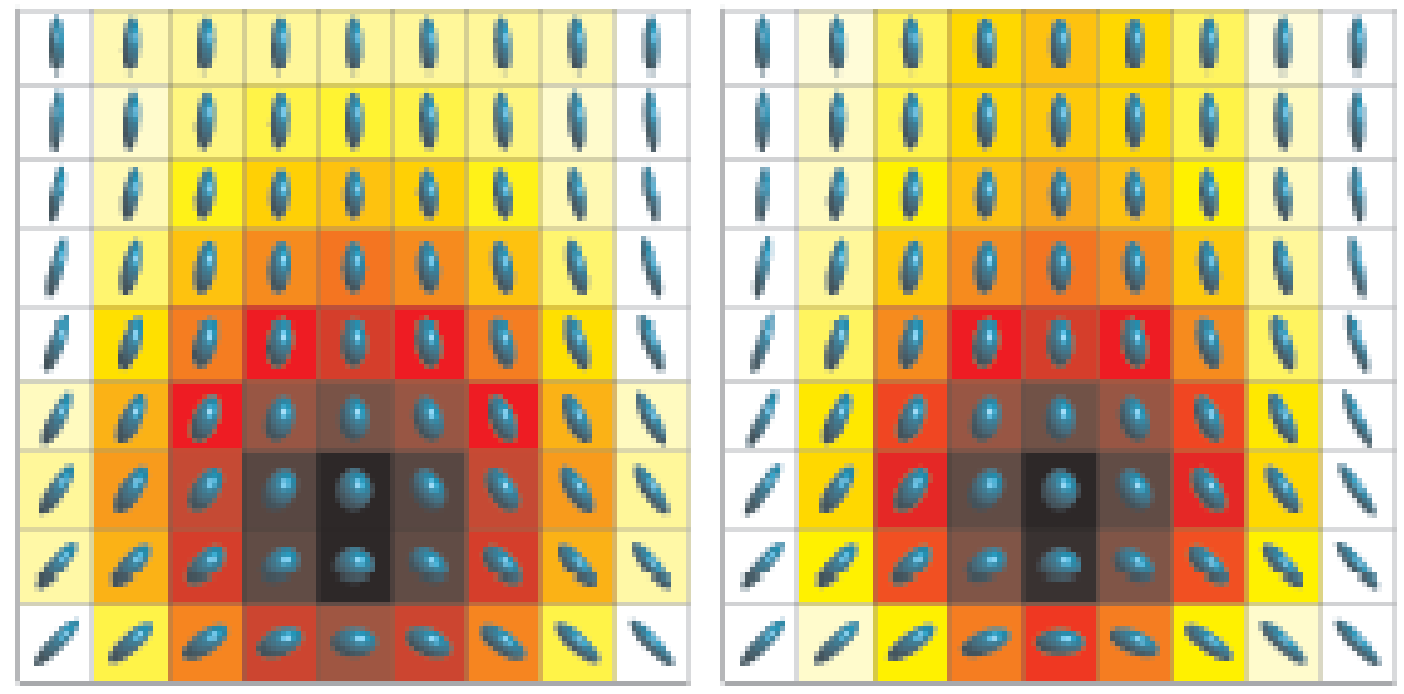

Figure 4.20 Bézier spline Interpolation, Rank 2 tensor results with Degree $\mathrm{n}=5$ (left) and $\mathrm{n}=6$ (right).
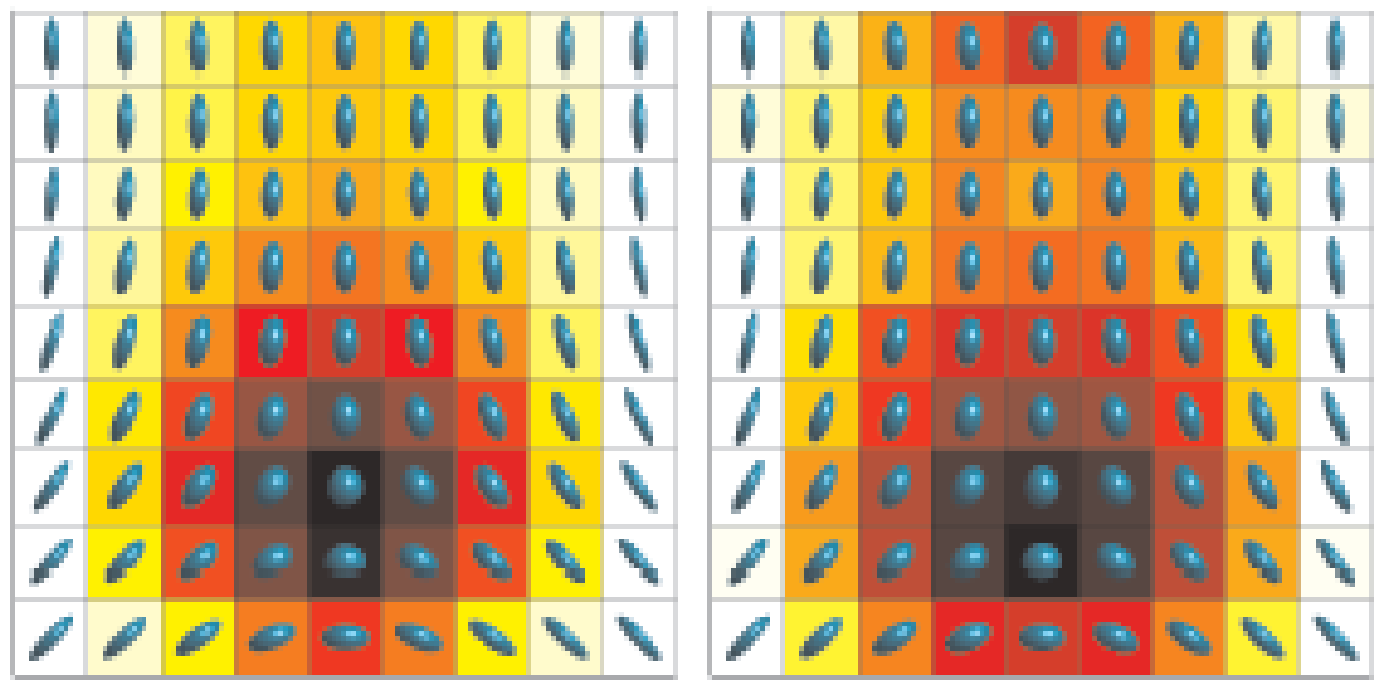

Figure 4.21 Bézier spline Interpolation, Rank 2 tensor results with Degree $\mathrm{n}=7$ (left) and Order 2 tensor subdivision results (right). 

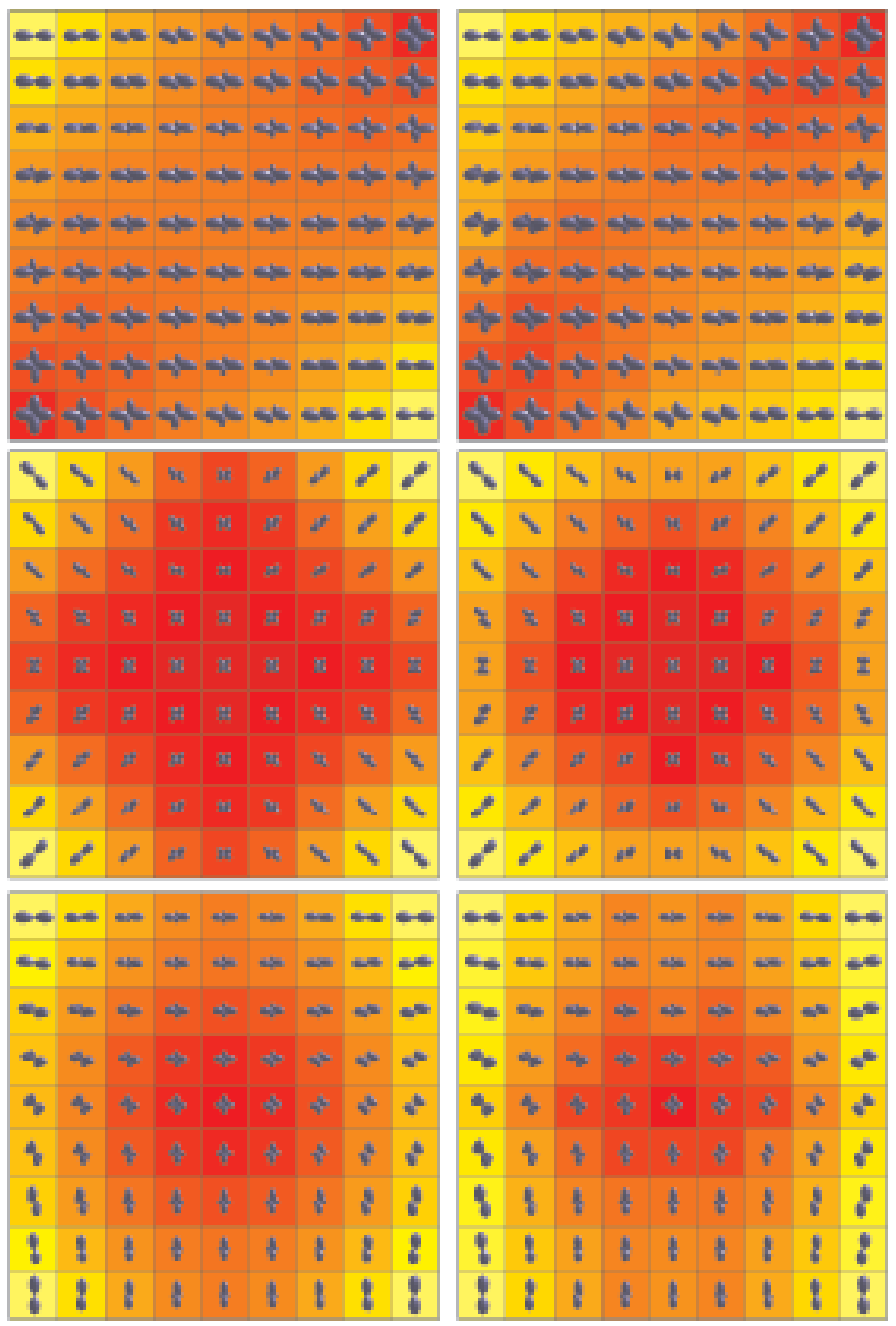

Figure 4.22 Rank-4 tensor interpolation, Degree $n=7$ (left) and subdivision (right). The background image is generalized anisotropy. 
Table 4.1 Slice average Frobenius Norm error for Subdivision and Linear Interpolation

\begin{tabular}{ccc}
\hline \hline Error & Subdivision & Linear Interpolation \\
\hline Max & $23.19 \%$ & $56.05 \%$ \\
Mean & $10.00 \%$ & $25,19 \%$ \\
Min & $0.0000 \%$ & $0.0000 \%$ \\
\hline
\end{tabular}

preserved during interpolation and the behavior of the subdivision solution is better approximated. In particular, the linear interpolation result has difficulty interpolating between the two tensors with perpendicular dominant orientations. The intermediate result is isotropic. Results for rank 4 tensor interpolation of synthetic data are shown in Figure (4.22). The background image represents generalized anisotropy (GA).

High angular resolution diffusion imaging can overcome some limitations or rank 2 diffusion tensor imaging. Models for the diffusivity function have been formulated in terms of tensors of various ranks [45], rank 4 tensors in particular [58] and sequences of tensors of increasing rank [69]. To demonstrate the generality of the subdivision scheme, we present the results of subdivision applied to rank 4 tensor fields in Figure (4.14), along with linear interpolation results.

In these examples it is apparent that the subdivision scheme encourages rotation in the peaks of the diffusivity profiles during interpolation. Note that these do not necessarily correspond to fiber directions. In order to determine fiber directions, we must compute the orientation distribution function from diffusivity. In the case of linear interpolation, the peaks in diffusivity merely grow and shrink while maintaining their orientation.

To verify the new subdivision algorithm, experimental results were obtained from datasets collected from a normal human volunteer, on a 3T Siemens Tesla General 


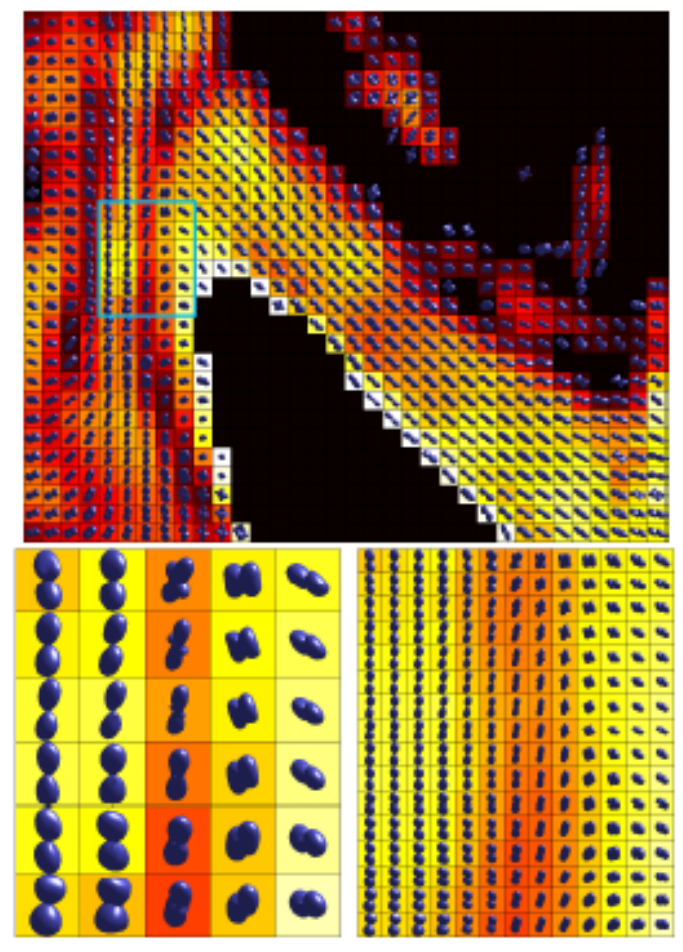

Figure 4.23 Interpolation of real data taken from sample within the blue box (top). Detail of the sample (bottom left), interpolated field (bottom right)

Electric Medical Systems Horizon LX imaging system with a diffusion weighted spin echo pulse sequence at bvalue $=1000 \mathrm{~s} / \mathrm{mm}^{2}$ for 25 directions and a single image was acquired with $b_{0}$ for 30 slices. The image field of view was $24 \times 24 \mathrm{~cm}$ and the image size was $256 \times 256$. The imaging parameters where imaging parameters were : effective $\mathrm{TR}=9000 \mathrm{~ms}, \mathrm{TE}=78 \mathrm{~ms}, \mathrm{NEX}=1$. Rank 2 tensors were calculated and then subdivision algorithm was then used. A sample of the results is shown in Figure (4.23). 


\subsection{Fiber Tracking and Shape Geometry}

The ability to non-invasively image the architecture of white matter pathways would greatly elucidate how neural signals in the human brain are coordinated and processed as part of a distributed network. While functional magnetic resonance imaging, positron emission tomography, and electromagnetic source imaging have shed considerable light on the anatomic location of the specialized processing regions within gray matter, there is currently no noninvasive imaging method capable of resolving the white matter connections between these regions [70-73].

Connectivity studies in animal brains are possible in vivo through a variety of invasive methods that cannot be used for humans [74]. Post mortem studies of fiber bundles are possible for human brains, for instance by observing passive diffusion using chemical dyes, but can take months to perform and are often affected by cross fiber diffusion [74]. Post mortem methods have revealed that white matter in the human brain is highly structured. However, using conventional MRI protocols, white matter appears to be homogeneous. Only recently, by the introduction of DT-MRI, in vivo studies of the human brain fiber tract anatomy have become possible. In DT-MRI the diffusion of water molecules is measured in different directions. This measure can be related to nerve fibers by the fact that water tends to diffuse only along fibers, because tightly packed myelin membranes restricts diffusion perpendicular to the axons. Myelin is not essential for anisotropic diffusion in fiber tracts, as shown in studies of nonmyelinated garfish olfactory nerves and neonates brains, but is widely assumed to be the main barrier for water diffusion [75]. The eigen vector corresponding to the largest eigenvalue of the diffusion tensor often give a good estimate of the local fiber orientation inside a voxel, as can be seen in Figure (4.24) bottom. A simple but effective method for fiber tracking is to simply follow the direction of 

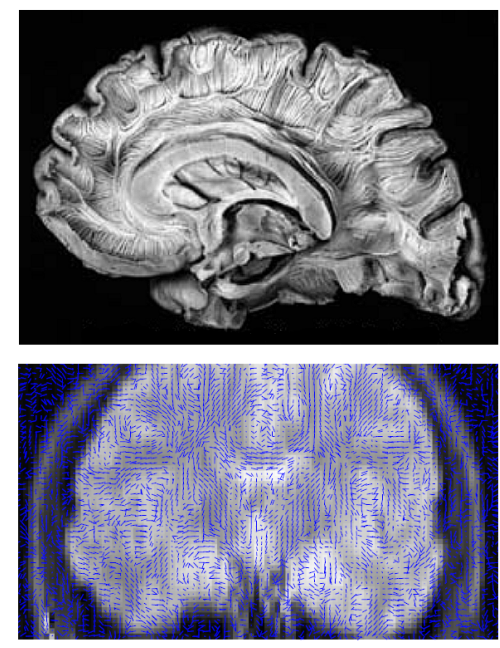

Figure 4.24 Top: A brain dissection showing the structure of white matter (from The Virtual Hospital, University of Iowa). Bottom: A coronal T2 slice combined with a plot of the eigenvector corresponding to the largest eigenvalue.

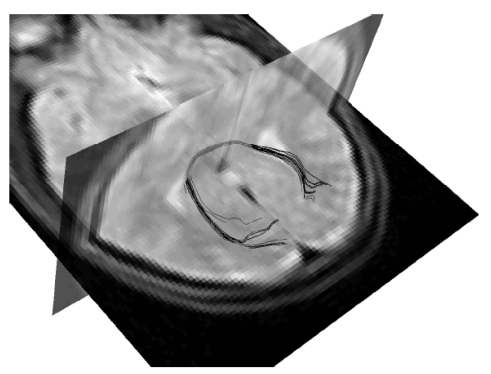

Figure 4.25 Tracking multiple fiber paths, following the direction of maximum diffusion. Visualized using the 3-D Viewer.

maximum diffusion, can be seen in Figure (4.25).

Many applications for white matter tractography and more will appear in the future as DT-MRI and fiber tracking becomes standard clinical procedures as [74]:

- Brain surgery may cause damage to important fiber bundles. Knowledge of their extension could minimize functional damage to the patient.

- White matter can be visualized using fiber traces for a better understanding of brain anatomy. 
- Connectivity between different parts of the brain can be inferred, which is useful for functional and morphological research on the brain.

- Understanding of diffusion properties in many brain-related diseases, e.g., multiple Sclerosis [76,77], Dyslexia [78], Alzheimers disease [79, 80], Schizophrenia $[81,82]$, brain tumours $[83,84]$, Periventricular Leukomalacia [85] as well as spinal cord injury [86] should benefit from those developments.

Most of the algorithms used to infer bundles of fibres from DT imaging are based on a discrete resolution of the integral curves of the vector field corresponding to the reduction of the diffusion tensor to its largest eigenvector as Fiber Assignment by Continuous Tracking Technique [74,87-90] and Streamline Technique [39, 40, 89,9193]. In Tensor Deflection (TEND), The tensor operator deflects the incoming vector towards the major eigenvector direction, but limits the curvature of the fiber [94-98]. Fast Marching Technique (FMT) is basically based on the representation of the front using scalar representation that allows control of front curvature and alignment to chosen features $[99,100]$. Although the tensor model provides a good description of a general diffusion profile, Friman al. [101] used the distribution of the fiber orientation stochastically for fiber tracking estimation.

The objective of this analysis is to find how the interpolation and the subdivision will affect the computed trajectory in comparison to fiber tracking without interpolation

\subsubsection{Fiber Assignment by Continuous Tracking Technique (FACT)}

The fiber tracking was initially introduced by Mori et al. [87] which was based on the principle of water-diffusion anisotropy. For a region where axons are aligned, water 
is restricted in the direction perpendicular to the axons and diffuses preferentially in a direction parallel to them. This situation can be represented mathematically by a so-called diffusion ellipsoid, characterized by diffusion constants along its three orthogonal directions and the (vector) direction of the longest axis [87], [102]. For example, (anisotropic diffusion) suggests the existence of cylindrical structures preferentially aligned along, whereas (isotropic diffusion) suggests sparse or unaligned axons. The most intuitive way to perform this tracking is by connecting each voxel to the adjacent one toward which the fiber direction is pointing [87]. However, when using this approach, the tracking often deviates from the true fiber orientation, because the choice of direction is limited to only eight angle ranges (26 in the case of 3D). This problem is avoided when tracking a continuous rather than a discrete vector field. The tracking is initiated from the center of a voxel and proceeds according to the vector direction. At the point where the track leaves the voxel and enters the next, its direction is changed to that of the neighbor. Due to the presence of continuous intercepts, this tracking now connects the correct voxels and the actual fiber can be assigned. The end point of the projection is judged based on the occurrence of sudden transitions in the fiber orientation [87].

Even though the 3D vector field obtained from the DTI consists of discrete voxels, the tracking is made in a continuous number field. Namely, a line is propagated from the center of the initial voxel along the direction of the vector until the line exits to the next voxel, as shown in figure (4.26). In this approach, the starting point in the next voxel is the intercept of the previous voxel. Once the line is propagated, voxels through which the line passes are connected to represent the fiber projection [102].

The tracking is terminated when it enters a region where the average of the inner products with the vectors of the three closest voxels is smaller than 0.75 . The tract is also terminated if the fractional anisotropy is very low, so that it represents a gray 


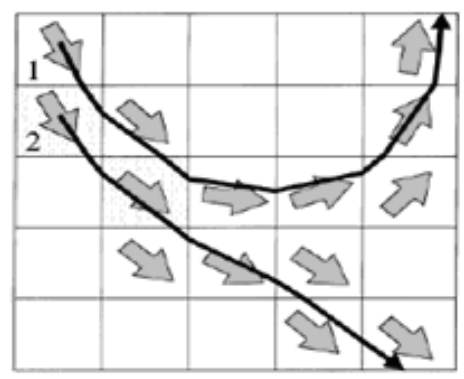

Figure 4.26 FACT Tracking Example

matter $[87,102]$.

In 2001, the same group [103] defined the minimum FA that describes how much the tract is valid, so if the $\mathrm{FA}$ is in range $0.25-0.35$, then the probability of tract validity is very low, and the inner product threshold of higher than 0.75 , identified trajectories by the FACT technique are likely to be valid.

Although the 3D FACT provides an exciting opportunity to visualize association pathways in vivo, its inherent limitations should also be realized. Specifically, fiber orientation revealed by DTI reflects the average orientation of axonal fibers for each pixel, and is susceptible to tissue heterogeneity. Within a pixel, numerous fibers may be crossing, or there may be a small portion of fibers that have different orientations from dominant fibers. Therefore, DTI fiber tracking is presently limited to visualization of in vivo gross anatomy of white matter tracts connecting functional brain regions $[104,105]$.

\subsubsection{Methods}

Two synthetic slices were generated, The first synthetic volume of isotropic material containing one third arc of a circle anisotropic fiber. The second volume is formed of isotropic material containing a spiral shaped fiber. The synthetic data is downsampled. Linear interpolation and subdivision is applied to the downsampled volume. 


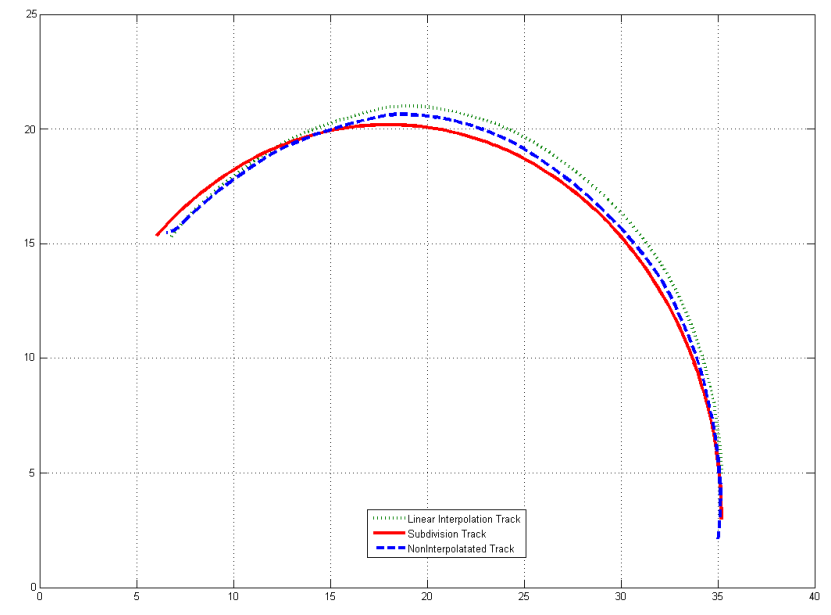

Figure 4.27 Circular Tract for the downsampled, Linearly Interpolated and the Subdivided volumes

FACT is then used for the noninterpolated downsampled volume, the linearly interpolated and the subdivided volume. The 3 generated tract are compared with the original tract. The mean and max radius error in case of the first synthetic is calculated.

\subsubsection{Results and Discussion}

The tracts for the synthetic volumes are shown in Figures $(4.27,4.28)$. It is clear from shown Table (4.27) that tracts for the interpolated and the subdivided are closer to the ground truth than the downsampled tract and that of the subdivided volume is the least deviated from the original tract. 


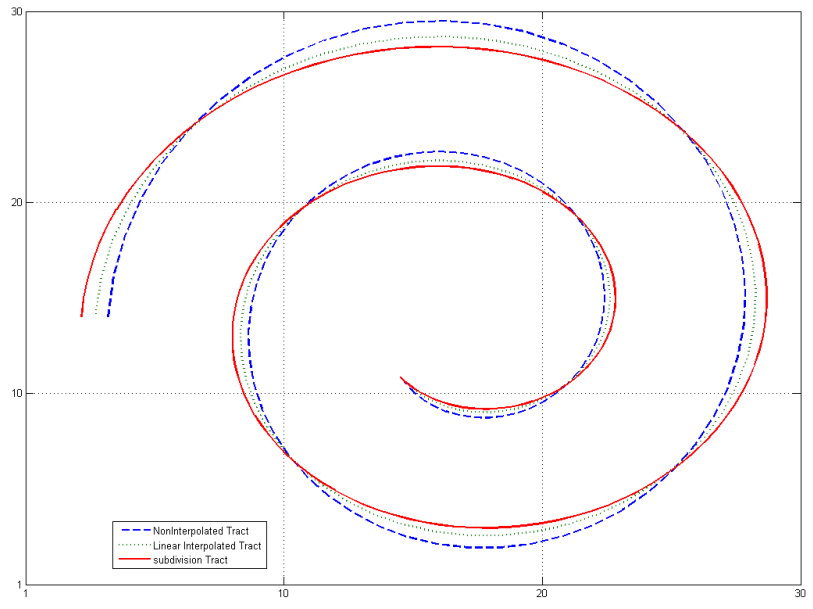

Figure 4.28 Spiral Tract for the downsampled, Linearly Interpolated and the Subdivided volumes

Table 4.2 Radius Error for the Circular tract

\begin{tabular}{ccc} 
Radius Error & Mean & Max \\
\hline Without Interpolation & $5.64 \%$ & $7.26 \%$ \\
Linearly Interpolated & $3.92 \%$ & $5.03 \%$ \\
Subdivision & $2.00 \%$ & $2.32 \%$ \\
\hline
\end{tabular}




\section{Chapter 5}

\section{New Measures Based on Divergence and Curl}

\subsection{Introduction}

In diffusion tensor MRI (DT-MRI) indices of anisotropy, as fractional anisotropy (FA), relative anisotropy [7], volume ratio [25], have found success in clinical applications. They are useful because many neurological disorders are characterized by changes in brain white matter anisotropy, for example stroke, trauma, and multiple sclerosis. Recently higher rank tensors have been proposed as a model for diffusion in the context of diffusion-weighted MRI [45]. Other measures of anisotropy based on variance and entropy have been proposed [46].

New scalar measures based on differential quantities can be computed from tensor fields of arbitrary rank. These studied quantities are generalizations of those which have proven useful in vector field analysis - namely the divergence and curl. The Helmholtz decomposition separates a flow field into divergence-free (solenoidal) and curl-free (irrotational) components. These parts may be analyzed separately to 
robustly identify different types of critical points in the field . The Helmholtz decomposition has recently proved to be useful in the topological analysis of vector fields. Polthier and Preuss [106] used a discrete Helmholtz decomposition to robustly locate singularities in vector fields. Li et al. [107] used the Helmholtz decomposition to segment 2D discrete vector fields. Tong et al. [108] described vector fields in a multiscale framework by defining a vector field scale space in terms of the separate scale spaces of the solenoidal and irrotational parts of the field. We apply a similar principal, and decompose the high rank tensor field into multiple components, and visualize each separately.

Local maxima of our scalar measures can be interpreted as topological features since they serve to identify generalized sources, sinks and vortices of the field. Several approaches to topological tensor field visualization have been described in previous literature. Many consider the topology of the dominant eigenvector field $[109,110]$ and define degenerate points as locations where two or more eigenvalues are equal to each other. Zheng et al. [111] described categories of feature points and numerically stable methods for extracting them and then joining them to form feature lines. Approaches specific to diffusion tensor MRI have considered the topology of scalar fields of tensor invariants as defined by crease lines. Tricoche et al. [112] use this framework applied to tensor mode (which is related to the skewness of eigenvalues), and Kindlmann et al. [14] used fractional anisotropy (which is related to the variance of eigenvalues). Another approach based on degenerate lines derived from probabilistic tractography has been described by Schultz et al. [15]. The concerns expressed in their work is the relying on eigenvectors or streamlines calculation as a preliminary step before the measurement calculation. The Helmholtz decomposition [113] of a vector field, $v$, is given by

$$
v=\nabla \phi+\nabla \times \psi+h
$$


where $\nabla \phi$ is the gradient of a scalar potential field $\phi, \nabla \times \psi$ is the curl of a vector stream field $\psi$ and $h$ is a harmonic vector field. Since $\nabla \phi$ is irrotational, so it is useful for identifying features such as local maxima and minima of divergence (foci of sources and sinks) in $v$ without interference from curl-based features. Likewise, $\nabla \times \psi$ is solenoidal, and is useful for isolating centers of vortices in $v$. The harmonic vector field, $h$, is both solenoidal and irrotational and typically is of small magnitude.

Using the previously defined operators, the Helmholtz decomposition can be extended to 2nd and 4th rank tensor fields as

$$
\begin{aligned}
D_{i j} & =\partial_{i} \phi_{j}+\varepsilon_{i m n}\left(\partial_{m} \psi_{n j}\right)+H_{i j}, \\
D_{i j k l} & =\partial_{i} \phi_{j k l}+\varepsilon_{i m n}\left(\partial_{m} \psi_{n j k l}\right)+H_{i j k l}
\end{aligned}
$$

Just as in the vector field case, the $\operatorname{div}(\operatorname{curl} \psi)=0$ and $\operatorname{curl}(\operatorname{grad} \phi)=0$. The formulation can be made for tensors of any arbitrary rank.

\subsection{Methods}

the discretized operators will be represented as block matrices where the blocks correspond to finite difference operators applied to a single tensor component. For 3D fields the multidimensional difference matrices are given by

$$
\begin{aligned}
& \Delta^{x}=I_{p \times p} \otimes I_{m \times m} \otimes \Delta_{n \times n}, \\
& \Delta^{y}=I_{p \times p} \otimes \Delta_{m \times m} \otimes I_{n \times n}, \\
& \Delta^{z}=\Delta_{p \times p} \otimes I_{m \times m} \otimes I_{n \times n}
\end{aligned}
$$

where $I_{n \times n}$ is an $n \times n$ identity matrix, $\otimes$ is the Kronecker product and $\Delta_{n \times n}$ is an $n \times n$ finite difference matrix. Central differences is used for approximating derivatives, in 
which case $\Delta$ is given by

$$
\Delta=\frac{1}{2}\left[\begin{array}{ccccc}
0 & +1 & 0 & \cdots & 0 \\
-1 & 0 & +1 & \ddots & \vdots \\
0 & -1 & 0 & \ddots & 0 \\
\vdots & \ddots & \ddots & \ddots & +1 \\
0 & \cdots & 0 & -1 & 0
\end{array}\right] .
$$

This definition of this matrix may be modified as needed to impose boundary conditions on the tensor field.

The curl of the second rank tensor field can be approximated as $\psi_{i j}$ as $\mathbf{C} \psi$, where

$$
\mathbf{C}=\left[\begin{array}{ccccccccc}
0 & 0 & 0 & -\Delta^{z} & 0 & 0 & \Delta^{y} & 0 & 0 \\
0 & 0 & 0 & 0 & -\Delta^{z} & 0 & 0 & \Delta^{y} & 0 \\
0 & 0 & 0 & 0 & 0 & -\Delta^{z} & 0 & 0 & \Delta^{y} \\
\Delta^{z} & 0 & 0 & 0 & 0 & 0 & -\Delta^{x} & 0 & 0 \\
0 & \Delta^{z} & 0 & 0 & 0 & 0 & 0 & -\Delta^{x} & 0 \\
0 & 0 & \Delta^{z} & 0 & 0 & 0 & 0 & 0 & -\Delta^{x} \\
-\Delta^{y} & 0 & 0 & \Delta^{x} & 0 & 0 & 0 & 0 & 0 \\
0 & -\Delta^{y} & 0 & 0 & \Delta^{x} & 0 & 0 & 0 & 0 \\
0 & 0 & -\Delta^{y} & 0 & 0 & \Delta^{x} & 0 & 0 & 0
\end{array}\right], \psi=\left[\begin{array}{c}
\psi_{x x} \\
\psi_{x y} \\
\psi_{x z} \\
\psi_{y x} \\
\psi_{y y} \\
\psi_{y z} \\
\psi_{z x} \\
\psi_{z y} \\
\psi_{z z}
\end{array}\right]
$$


Similarly, the gradient of the first rank tensor field $\phi_{i}$ is given by $\mathbf{G} \phi$ where

$$
\mathbf{G}=\left[\begin{array}{ccc}
\Delta^{x} & 0 & 0 \\
0 & \Delta^{x} & 0 \\
0 & 0 & \Delta^{x} \\
\Delta^{y} & 0 & 0 \\
0 & \Delta^{y} & 0 \\
0 & 0 & \Delta^{y} \\
\Delta^{z} & 0 & 0 \\
0 & \Delta^{z} & 0 \\
0 & 0 & \Delta^{z}
\end{array}\right], \phi=\left[\begin{array}{c}
\phi_{x} \\
\phi_{y} \\
\phi_{z}
\end{array}\right]
$$

The discretized operators for rank-4 tensors will contain 81 rows each.

To perform the generalized Helmholtz decomposition we solve the least squares problem

$$
\min _{\psi, \phi}\|D-\mathbf{C} \psi-\mathbf{G} \phi\|_{F}^{2}
$$

where $\|\cdot\|_{F}$ denotes the Frobenius norm of the tensor $\left\|X_{i k}\right\|_{F}=\operatorname{Trace}\left(X_{i j} X_{j k}\right)$.

Using the fact that $\mathbf{C}^{T} \mathbf{G}=\mathbf{G}^{T} \mathbf{C}=0$, numerical implementation is used by alternately solving the normal equations

$$
\begin{aligned}
\mathbf{C}^{T} \mathbf{C} \psi & =\mathbf{C}^{T} D, \\
\mathbf{G}^{T} \mathbf{G} \phi & =\mathbf{G}^{T} D
\end{aligned}
$$

using a stabilized biconjugate gradients method until convergence is reached. Although the matrices on the left-hand sides of Equation (5.8) are symmetric, they are not positive-definite, so the standard conjugate gradients method cannot be used. The derivatives of all tensor components are constrained to be zero across each boundary. Since, the objective is not explicitly solving for $H$, the harmonic part of the field, but instead let $H=D-$ Grad $\phi-C u r l \psi$. 

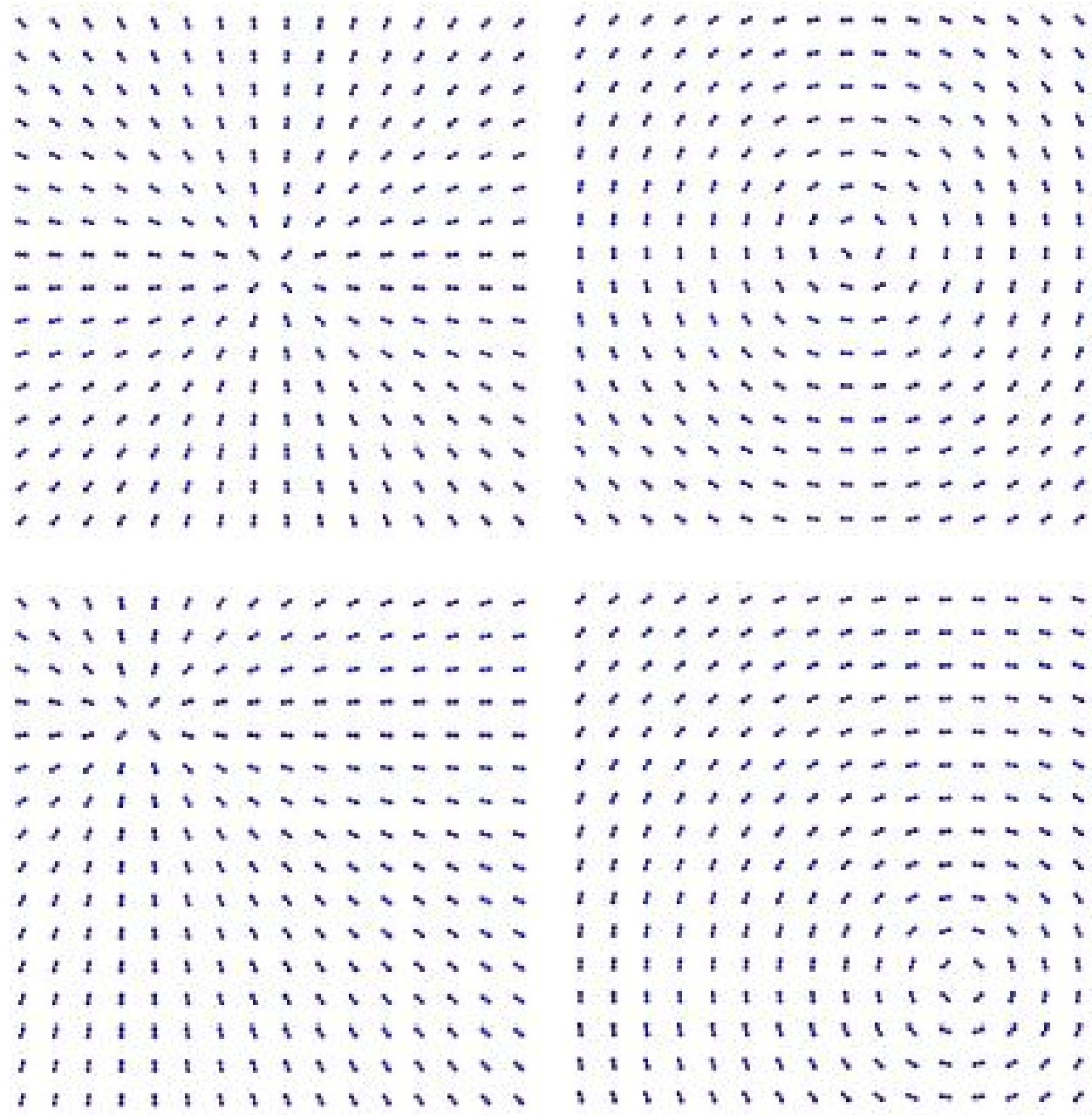

Figure 5.1 Vortices and sources used to construct the synthetic field.

\subsection{Results}

A synthetic rank-2 tensor field was generated from the sources and vortices shown in Figure (5.1) by computing $D=\left(D_{1}+D_{2}+D_{3}+D_{4}\right)^{2}$. The tensor fields in Figures $(5.1,5.2)$ are visualized by plotting the radial surfaces $r(x)=D_{i j} x_{i} x_{j}$ for unit vectors $x$. The surface is colored blue when $r$ is positive and red when $r$ is negative. The results of the generalized Helmholtz decomposition are shown in Figure (5.2).

Another synthetic tensor field was generated from sources and vortices similar to those shown in Figure (5.1), but modeled as rank-4 tensors. The results of the 
- $88 \delta^{\circ} 0^{\circ} 0000000000$

- 18 \& 800000000000

- I \& \& 800000000000

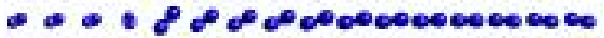

$-\infty<1 .+\infty 00000000000$

- \& \& 8 \& 10000000000

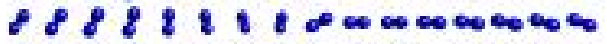

\&888 8 8 $=00000000$

$8888881.80000000 \%$

$828888814000000 \%$

888888881000088

$88888888881=888$

$888888888888 \ldots$

$88888888888 \% \cdots \cdots$

$888888888840 \% \cdots$

$218828248480 * 0$
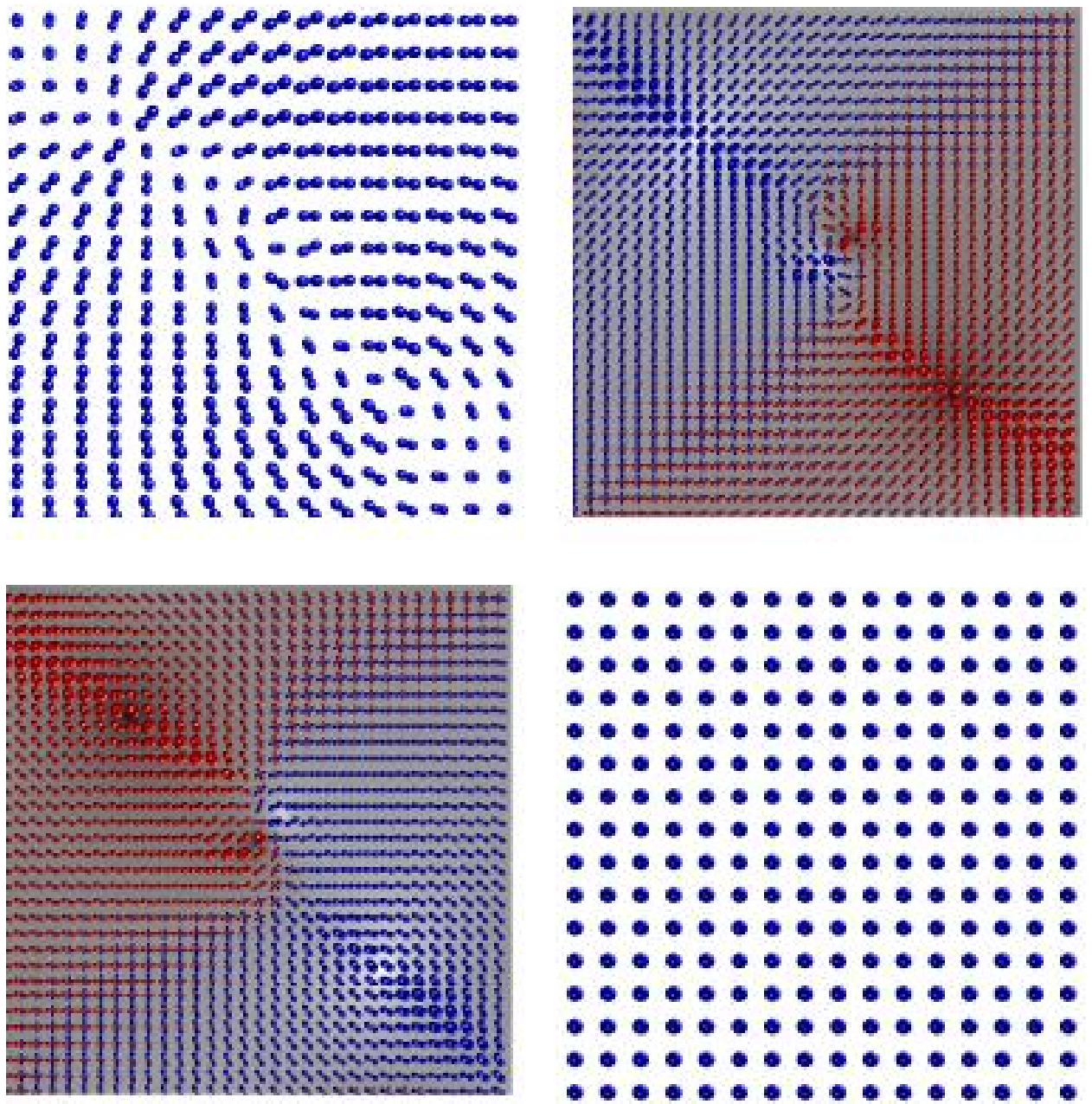

Figure 5.2 Helmholtz decomposition results for rank-2 synthetic tensor field.[top: right: Tensor, Left: Curl, Bottom: right:, left: Harmonic] 
generalized Helmholtz decomposition of this field are shown in Figure (5.3), the background of Figure $(5.3 \mathrm{~b}, \mathrm{c})$ is the trace of the tensor. The tensor fields are visualized by plotting the radial surfaces $r(x)=D_{i j k l} x_{i} x_{j} x_{k} x_{l}$ for unit vectors $x$. The surface is colored blue when $r$ is positive and red when $r$ is negative. Several interesting observations can be made from these results. The critical points in the original field Figure (5.2a), are not clearly visible, but in the decomposed fields they are quite evident. In the decomposed fields there seems to be a correspondence between sources of positive-definite tensors and vortices of negative-definite tensors. The harmonic field, which is typically of small magnitude for vector field decompositions, can be substantial in terms of the tensor trace, but it is extremely smooth - nearly constant in all of our synthetic field experiments.

The decomposition was also applied to diffusion tensor MRI of the human brain described in section 4.2.5. Rank 2 and 4 tensors were computed from the diffusion weighted images by performing a least squares fit to the logarithm of the signal attenuation.

Denoting the irrotational part of the field as $D_{\phi}=G r a d \phi$ and the solenoidal part as $D_{\psi}=$ Curl $\psi$, images of $\left\|\operatorname{Div}\left(D_{\phi}\right)\right\|$ and $\left\|C u r l\left(D_{\psi}\right)\right\|$ in Figures $(5.6,5.3)$ are shown. Images of fractional anisotropy [24] are also presented for comparison. The new scalar measures are based on differential operators applied to tensor fields generated by a global optimization procedure, unlike FA which is simply computed on a voxel-by-voxel basis. As such, these new measures are sensitive to the large changes in diffusivity which occur at the cortical surface and the boundaries of the ventricles. Away from these boundaries it is clear that the critical points do form coherent linear and curved regions in the field, as predicted by previous work.

Compared to FA, new measures seem to be more discriminative, often revealing thinned structures. This can be understood in relation to vector field topological 

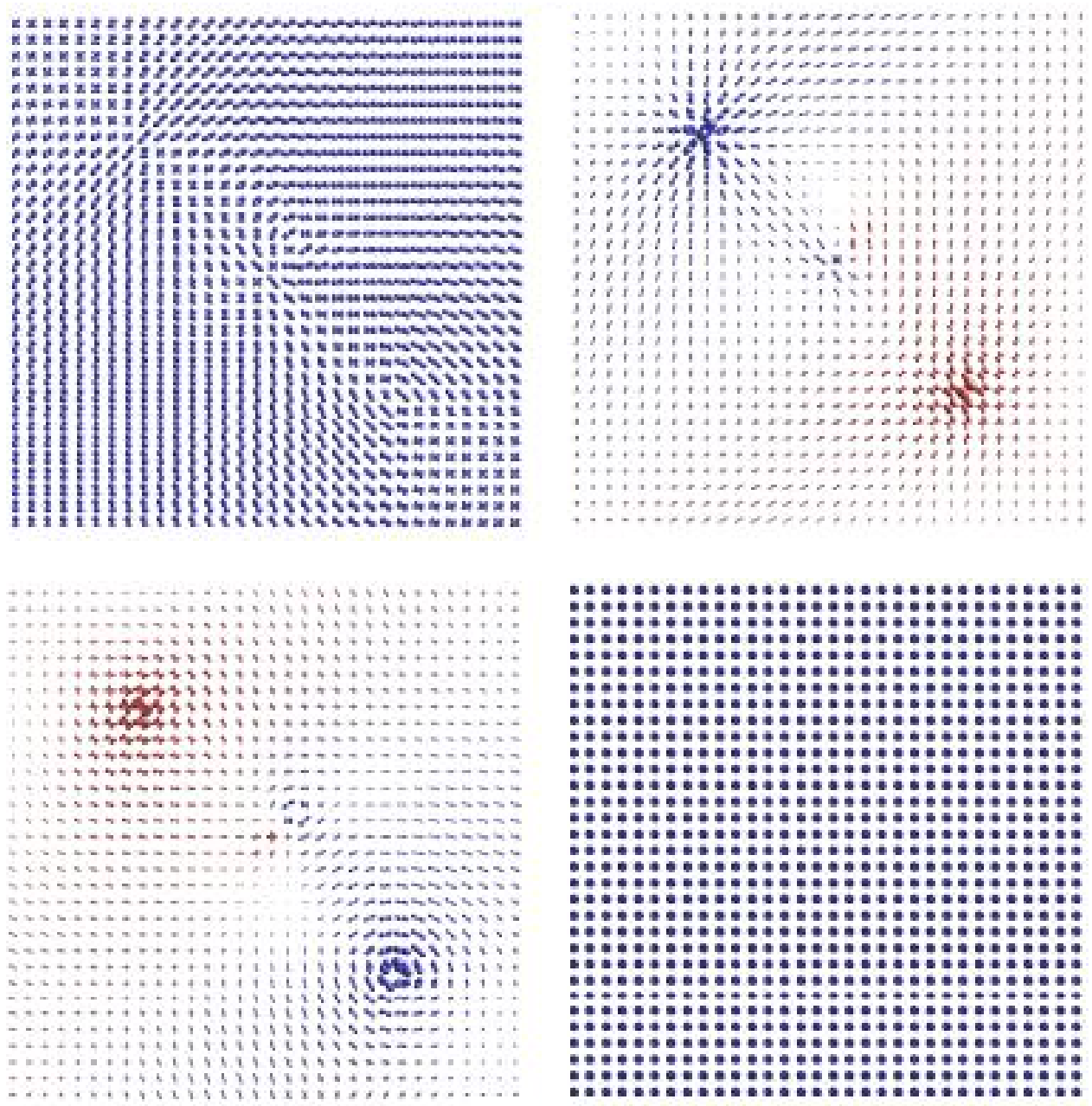

Figure 5.3 Helmholtz decomposition results for rank-4 synthetic tensor field.[top: right: Tensor, Left: Curl, Bottom: right:, left: Harmonic]
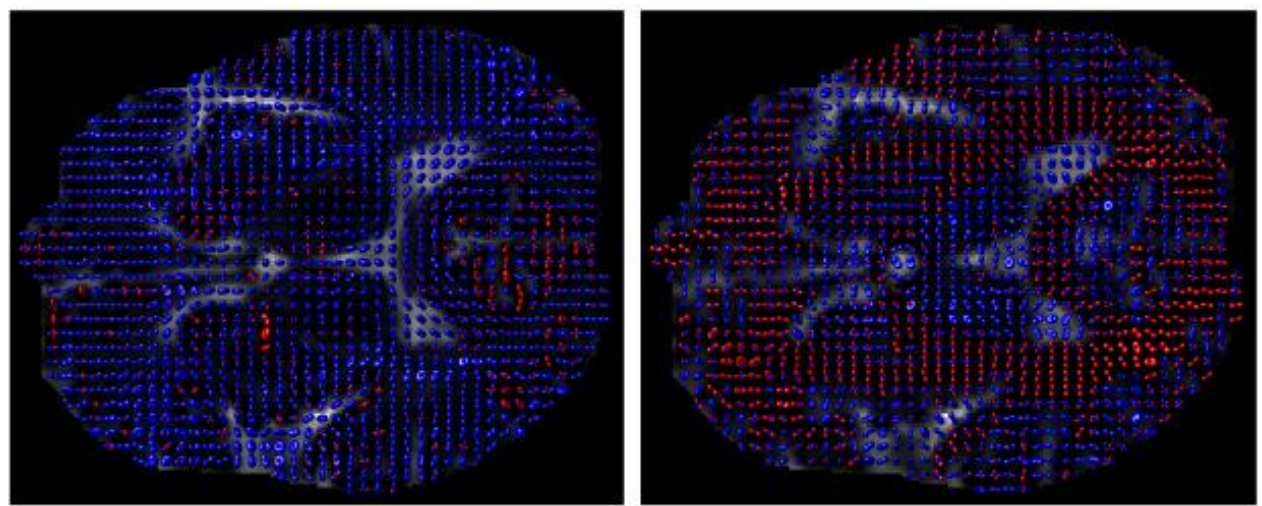

Figure 5.4 Axial slice of real data, rank 2. [left:Curl $\psi$, Right: Grad $\phi]$. 

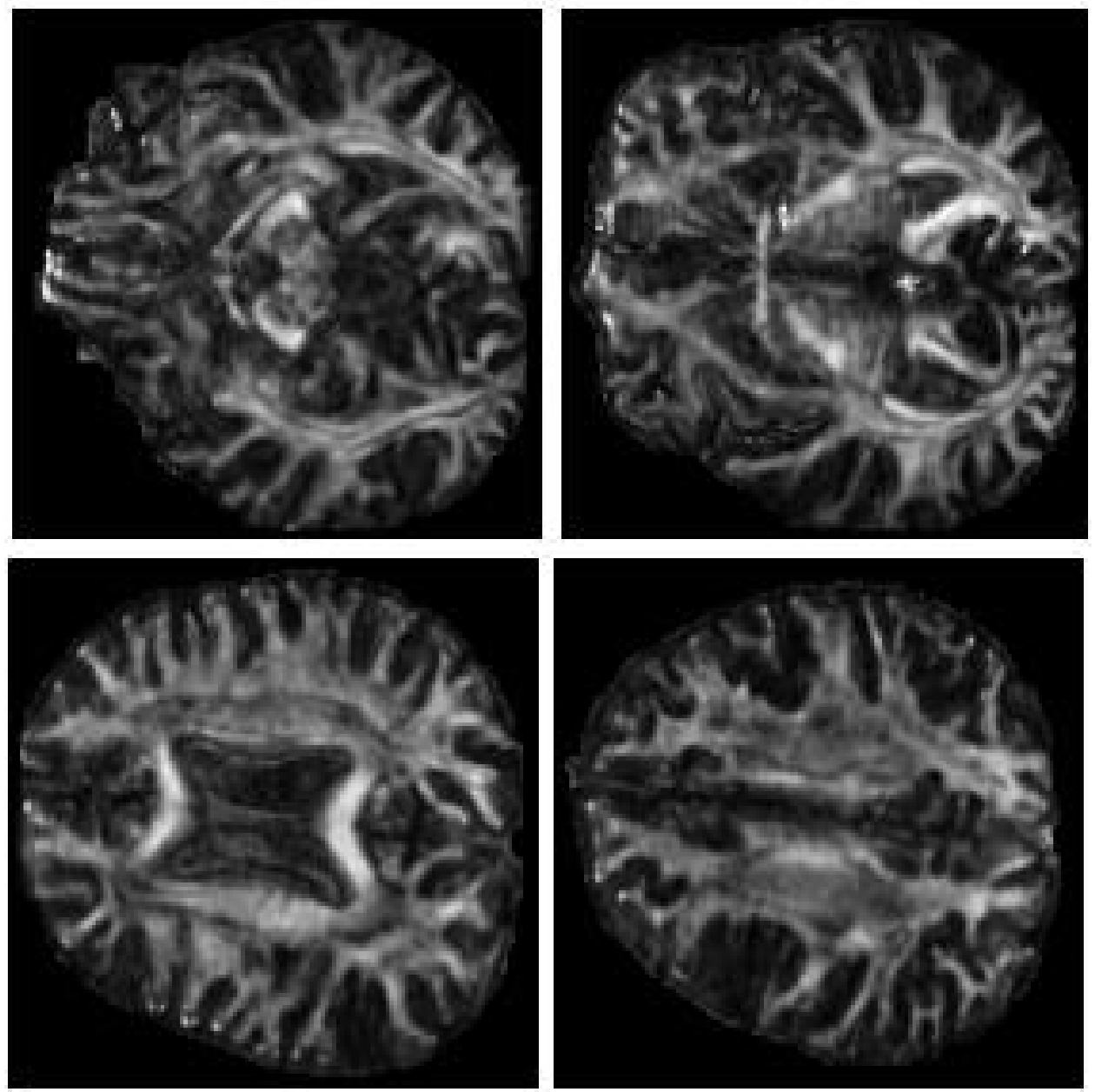

Figure 5.5 A comparison of FA of white-matter structure for an axial slice . ILF: interior longitudinal fasciculus, SFO: superior fronto-occipital fasciculus, SCC: splenium of corpus callosum, RCB/LCB: right/left cingulum bundle, ATR: Anterior thalamic radiation. 

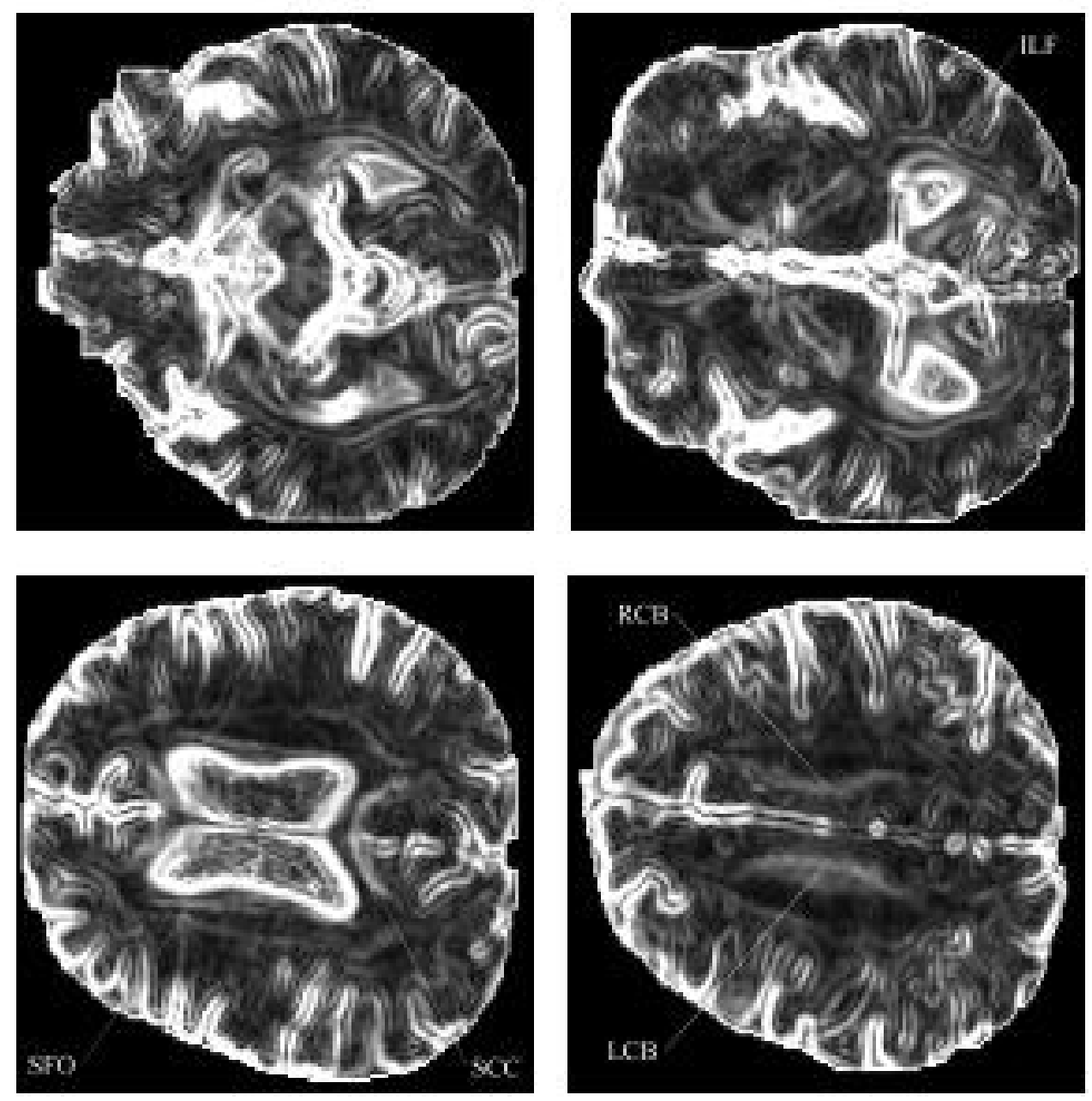

Figure 5.6 A comparison of $\left\|C u r l D_{\psi}\right\|$ of white-matter structure for an axial slice. ILF: interior longitudinal fasciculus, SFO: superior fronto-occipital fasciculus, SCC: splenium of corpus callosum, RCB/LCB: right/left cingulum bundle, ATR: Anterior thalamic radiation. 

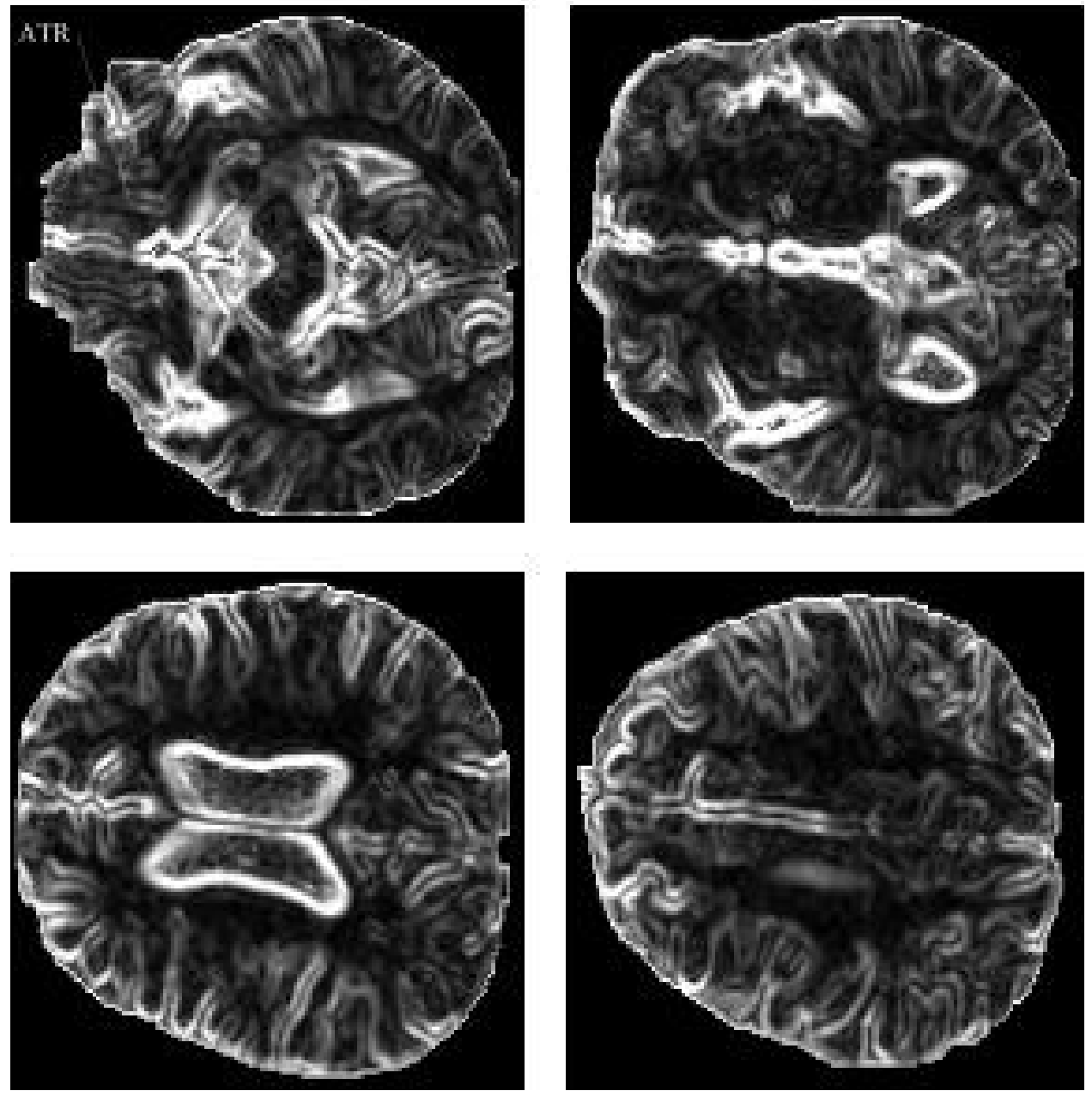

Figure 5.7 A comparison of $\left\|D i v D_{\psi}\right\|$ of white-matter structure for an axial slice. ILF: interior longitudinal fasciculus, SFO: superior fronto-occipital fasciculus, SCC: splenium of corpus callosum, RCB/LCB: right/left cingulum bundle, ATR: Anterior thalamic radiation. 

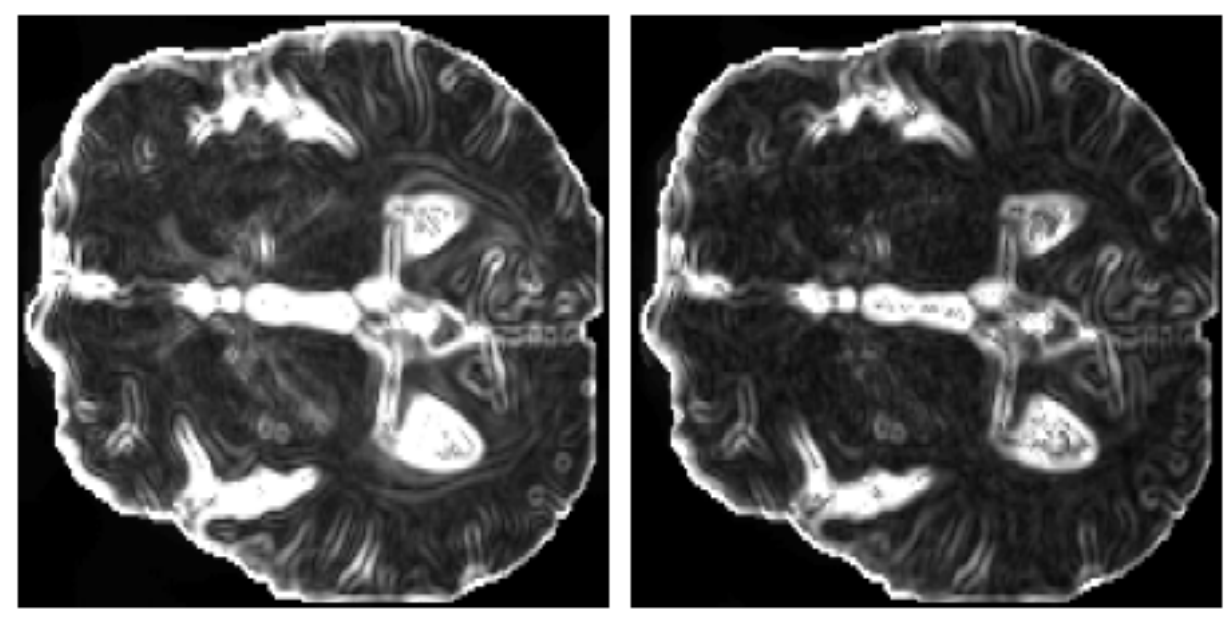

Figure 5.8 Scalar measures of the rank-4 tensor field for an axial slice of real huma brain.[left: $\left\|D_{\psi}\right\|$, Right: $\left.\left\|D_{\psi}\right\|\right]$

visualization technique which use divergence and curl to locate centers of features such as vortices, sources and sinks. These form line structures in 3D flows, and we see analogous behavior from the generalized measures in 3D tensor fields. In the bottom image of Figure (5.6) the left and right cingulum bundles are visible as a pair of bright horizontal regions. We note that the curl image seems to convey much more structural information than the divergence image. This may be due to the incompressibility of water resulting in smaller fluctuations in divergence.

Results for rank-4 tensors computed from the same diffusion weighted data as above are presented in Figure (5.8). The displayed slice is the same as the second column from the left in Figure (5.6). 


\section{Chapter 6}

\section{Orientation Distribution Function}

\section{and Anisotropy Measure}

In order to visualize and analyze the diffusion function, Tuch [36] used the idea of projecting the diffusion function on to the sphere, and the resulting function was termed a spin displacement Orientation Distribution Function (ODF). The diffusion function within each voxel was reconstructed by sampling the diffusion signal on a Cartesian grid and then taking the Fourier transform on the grid.

The radial projection used to construct the ODF discards all of the radial information contained in the diffusion function. Hence, the ODF does not contain the radial information which was originally present in the Cartesian diffusion function, but preserves the salient angular contrast. It would therefore dramatically boost the acquisition efficiency, to measure the ODF directly and bypass the intermediate reconstruction of the diffusion function, much of which is ultimately discarded by the radial projection. The above goal could be accomplished by a sampling/ reconstruction scheme which sampled the diffusion signal directly on the sphere and reconstructed the ODF directly on the sphere. 
The ability to sample directly on the sphere would allow to spend the signal acquisitions more efficiently on angular resolution. In contrast, the signal acquisitions in conventional Cartesian Fourier reconstruction are invested in spatial resolution which contributes relatively inefficiently to the desired end goal of angular resolution.

Moreover, spherical sampling provides a more natural framework for describing angular resolution. Whereas the angular resolution of a spherical sampling scheme is upper-bounded by the angular distance between the sampling points, it is not clear how to define angular resolution in the context of Cartesian sampling. Additionally, with spherical sampling the acquisition can be targeted to the spatial frequency band where the angular contrast-to-noise is greater.

However, the reconstruction involved an elaborate model-fitting procedure which was prone to model mis-specification. An independent sampling model and reconstruction scheme termed Q-ball imaging is used that samples the diffusion signal directly on the sphere, and reconstructs a function closely resembling the ODF obtained from explicit radial projection.

Based on the classical diffusion ODF reconstructed from QBI and the very recent regularized version of the diffusion ODF, a streamline approach with curvature constraint following all maxima to deal with fibers crossing has been proposed by Descouteaux et al. [114,115]. Behrens et al. [116] used the Markov Chain Monte Carlo (MCMC) for Bayesian Fiber tracking, but it suffers from being very time consuming.

Jones et al. [117] used nine redundant sets of DWI volumes obtained to perform the Bootstrap method.

In this chapter, different methods to calculate the tensor distribution function and the anisotropy measure based on this distribution will be introduced, and finally a novel anisotropy measure will be introduced. Some experimental results will be shown to prove the validity of the measure, and then used at the end on a DTI Brain 
data set to compare with other measures.

\subsection{Introduction}

A single second order tensor model is incapable of resolving multiple fiber orientations within an individual voxel. This shortcoming of the tensor model stems from the fact that the tensor possesses only a single orientational maximum, i.e., the major eigenvalue of the diffusion tensor. At the millimeter-scale resolution typical of DTI, the volume of cerebral white matter containing such intravoxel orientational heterogeneity (IVOH) may be considerable given the widespread divergence and convergence of fascicles. The abundance of IVOH at the millimeter scale can be further appreciated by considering the ubiquity of oblate (pancake-shaped) diffusion tensors in DTI, a hypothesized indicator of IVOH.

Tissues with regularly ordered microstructure such as skeletal muscle, spine, heart, and cerebral white matter exhibit anisotropic (that is, directionally-dependent) water diffusion due to the preferred orientation of the diffusion compartments in the tissue. In order to avoid non-unique variations in measuring diffusivity parameters with the positioning of the subject, a more general characterization of the diffusion process was introduced based on diffusion tensors. The basic techniques in diffusion tensor imaging attempt to characterize the 3-D diffusion phenomena in terms of a 3-D Gaussian probability distribution [8]. Based on the eigen structure of the measured diffusion tensor it is possible to infer the orientation of the diffusion compartments within the voxel. Although the success of DTI for resolving the mean fiber orientation in tissue, the tensor model is incapable of resolving multiple fiber orientations within an individual voxel. However, such representation is sufficient in case of the so-called cigar-shaped diffusion tensor representation. 


\subsection{Literature Review}

To overcome this limitation of diffusion tensor model, a number of advanced image acquisition strategies and sophisticated mathematical models have been proposed. With the multidirectional measurements from the high angular resolution diffusion weighted imaging (HARDI) method [118]. However, one major difficulty with employing HARDI in studies involving orientation mapping has been that the peaks of the diffusivity profile do not necessarily yield the orientations of the distinct fiber populations. Orazlan et. al [45] have shown that the (SHT) approach could be seen as a generalization of DTI since the coefficients of the Laplace series (obtained from the SHT of the diffusivity profile) are related to the components of higher-order Cartesian tensors. In this section, some of the ODF Algorithms will be discussed.

\subsubsection{Diffusion Spectrum Imaging (DSI)}

This methodology image a distribution of fiber orientations within each voxel as a 3D probability density function (PDF) of proton diffusion with Q-space diffusion MRI using Fourier Transform (FT) encoding and reconstruction. A disadvantage of this algorithm is its insensitivity to asymmetric internal spin motion such as perfusion and streaming or effective motion produced by asymmetric relaxation sinks, i.e. this methodology will detect the spatially symmetric part of such motion, however more complex transport effects are also possible [37]. Diffusion-weighted single-shot echo-

planar NMR images are acquired for several hundred values of the diffusion-encoding spatial modulation q comprising points of an isotropic 3D grid contained within a spherical volume of radius $\mathrm{r}$. At each voxel, the signal data $\mathrm{S}(\mathrm{q})$ comprise a sampling of the 3D Fourier transform of the probability density function of spin translation

$$
P(\Delta r)=<P(y \mid y+\Delta r, \tau)>
$$


where $\langle P(y \mid y+\Delta r, \tau)>$ is the conditional displacement probability density from location $y$ to point $y+\Delta r$ at the given mixing time $\tau$ and the angle-bracket denotes an average over the observed spins within a voxel. Then $P(\Delta r)$ in each voxel is the inverse 3DFT of the signal

$$
P(\Delta r)=F[S(q)]
$$

Based on this representation, diffusion tensor MRI can be viewed as a second-order approximation of a 3D displacement probability distribution that can be imaged in full with Fourier methods [37].

\subsubsection{Persistent Angular Structure(PAS)}

The (radially) persistent angular structure (PAS) represents the relative mobility of particles in each direction. PAS uses a method based on the principle of maximum entropy. The information content of $\mathrm{p}$ is defined by [119]:

$$
I(p)=\int_{\Omega} p(x) \ln (p(x)) d x
$$

To extract useful information about the angular structure in a computationally efficient way, the authors restricted attention to determining a probability density function of the form

$$
p(x)=\tilde{p}(\hat{x}) r^{-2} \delta(|x|-r)
$$

where $\delta$ is the standard one-dimensional $\delta$ distribution, $r$ is a constant and $\hat{x}$ is a unit vector in the direction of $x$. it was just the projection of the angular structure from all radii onto the sphere of radius $r$, and ignoring any information about the radial structure in the data, which is often very limited. The final result is weakly dependent on the choice of $r$. The PAS $\tilde{p}$ has a unit sphere domain as it represents only orientational information. The relative entropy of the probability density function $p$ 
with respect to the probability density function $p_{0}$ is given by

$$
I\left[p ; p_{0}\right]=\int_{\Omega} p(x) \ln \left(\frac{p(x)}{p_{0}(x)}\right) d x
$$

The constraints on $p$ from the data can be incorporated into the expression above using the method of Lagrange multipliers to yield

$$
I\left[p ; p_{0}\right]=\int\left(\tilde{p}(\hat{x}) \ln (\tilde{p}(\hat{x}))-\tilde{p}(\hat{x}) \sum_{i=1}^{N}\left(\lambda_{j} \exp \left(i q_{j} \cdot r \hat{x}\right)\right)-\tilde{p}(\hat{x}) \mu\right) d \hat{x}
$$

where $q_{j}, 1 \leq j \leq N$, are the non-zero wave numbers for the MRI measurements, the $\lambda_{j}$ are Lagrange multipliers for the constraints from the data and the Lagrange multiplier $\mu$ controls the normalization of $\tilde{p}$ The expression was finally simplified to

$$
\tilde{p}=\exp \left(\lambda_{0}+\sum_{j=1}^{N} \lambda_{j} \cos \left(q_{j} \cdot r \hat{x}\right)\right)
$$

PAS extracts the directional information from the DTI data, which may be small and will often be restricted to a sphere in Fourier space. The advantage of this approach is its statistic robustness and its correspondance to the physiological structure of the human brain.

\subsubsection{Diffusion Orientation Transform (DOT)}

Orszlan et. al [120] used The Fourier transform to relate the signal attenuation to the water displacement probability in spherical coordinates. They use the HARDI data to estimate the probability of finding the particle at the point $R_{0} r$ away from the origin involving the following steps:

- Compute the diffusivity $D(u)$ along each direction $\mathrm{u}$.

- Compute the radial integral $I_{l}(u)$, where $I_{l}(u)$

$$
I_{l}(u)=4 \pi \int_{0}^{\infty} j_{l}\left(2 \pi q R_{0}\right) \exp \left(-4 \pi^{2} q^{2} t D(u)\right) d q
$$

where $j_{l}\left(2 \pi q R_{0}\right)$ is the $l^{\text {th }}$ order spherical Bessel function. 
- For each 1 , compute $\alpha_{l l m}$, the $l_{t h}$ order spherical harmonic transform of $I_{l}(u)$, which will be described in section 6.4.1, using the equation (6.9):

$$
\alpha_{l l^{\prime} m^{\prime}}=\int Y_{l^{\prime} m^{\prime}}(u) I_{l}(u) d u
$$

where $Y_{l m}$ is the spherical Harmonic function 6.4.1

- Evaluate the three dimensional displacement probability function

$$
p\left(R_{0} r\right)=\sum_{l=0}^{\infty} \sum_{m=-l}^{l} p_{l m} Y_{l m}(r)
$$

where $p_{l m}=(-i)^{l} \alpha_{l l m}$

This technique provides a a robust and fast direct estimation of displacement probability surfaces within each voxel. DOT can easily construct high resolution probability surfaces from the signal values. When the acquisition time or the available gradient strength is limited, the monoexponentiality assumption can be employed. This results in some broadening of the PDF whose angular structure is smoother.

\subsubsection{Tensor Distribution Function}

The algorithm's objective is to calculate a probabilistic ensemble of tensors, as represented by a tensor distribution function (TDF) $P^{*}$. To solve for an optimal TDF, multiple diffusion-sensitized gradient directions $q_{i}$ and arrive at $P^{*}$ using the leastsquares principle

$$
P^{*}=\operatorname{argmin}_{p} \sum_{i}\left(S_{\text {observed }}\left(q_{i}\right)-S_{\text {calculated }}\left(q_{i}\right)\right)^{2}
$$

this is used to define the error vector $E\left(q_{i}\right)=S_{\text {observed }}\left(q_{i}\right)-S_{\text {calculated }}\left(q_{i}\right)$ to be the contribution to the total error with respect to $q_{i}$. For $\mathrm{P}(\mathrm{D})$ to be a true TDF, two constraints have to be enforced, 
- The non-negativity constraint: $P(D) \geq 0$ for every $\mathrm{D}$, and this is enforced by using the non-negativity property of the exponential function $P(D)=\exp (R(D))$

- The probability density constraint: $\int P(D) d D=1$

The gradient descent will be solve the minimization problem in the $\mathrm{R}$ space. Once the optimal TDF is calculated, the displacement probability function $p$ is simply:

$$
p(x)=\int_{D \in R} P(D)\left((4 \pi t)^{3} \operatorname{det}(D)\right)^{\frac{-1}{2}} \exp \left(-\frac{X^{T} D^{-1} x}{4 t}\right) d D
$$

The ODF can then be computed analytically using the following equation:

$$
O D F(\tilde{x})=C \int_{r=0}^{\infty} p(r \tilde{x}) d r=C \int_{D \in D} P\left(\operatorname{det}(D) \tilde{x}^{T} D^{-1} \tilde{x}\right)^{\frac{-1}{2}} d D
$$

where $\mathrm{C}$ is a normalization constant. The TDF approach can be considered a hybrid methodology as it has theoretical similarities to other approaches. In TDF, a weight is being assigned to any tensor whose anisotropic properties are consistent with human physiology (i.e., in the solution space). By using the logarithmic transform along with projected gradient descent, the TDF algorithm naturally yields positive weights for all tensors in the solution space, without the need for extra constraints or numerical procedures. but Still the-art analytic ODF reconstruction methods through spherical harmonics are much faster and require fewer directions [121].

\subsubsection{Q-Ball Imaging (QBI)}

Q-Space imaging was introduced firstly by Tuch [122] as the Fourier transformation of the diffusion signal to measure the diffusion function directly, without recourse to a model of the diffusion process, but it was found that it only gives the diffusion PDF exactly when there is no appreciable diffusion during the diffusing encoding period. QSI employs the Fourier relation between the diffusion signal and the diffusion function. The QSI technique requires gradient sampling on a three-dimensional Cartesian 
lattice, which is time intensive and it requires large pulsed field gradients to satisfy the Nyquist condition for diffusion in nerve tissue.

A completely model-free reconstruction scheme for HARDI QBI was then introduced, where The reconstruction is based on a spherical tomographic inversion called the Funk Radon transform (FRT) which is the extension of the original FRT to map from three-dimensional Cartesian space to the sphere, which is defined as the FRT evaluated at a particular radius $r^{\prime}$. Given a three-dimensional function $f(x)$, where $\mathrm{x}$ is a three-dimensional vector [122].

It is substantially more efficient to reconstructing the ODF directly using spherical sampling and reconstruction has a number of advantages:

- both the sampling and the reconstruction are both performed on the sphere so the reconstruction is immune to Cartesian reconstruction bias

- With a spherical sampling scheme, there is also a natural framework for calculating the angular resolution, whereas it is not clear how to define the angular resolution for a Cartesian scheme

- The acquisition can be targeted to specific spatial frequency bands of interest by specifying the radius of the sampling shell

The authors related the ODF and the FRT using a PDF in cylindrical coordinates as $P(r, \theta, z)$.

$$
\psi(\mathbf{u})=G_{q^{\prime}}[E(q)]=2 \pi q^{\prime} \int P(r, \theta, z) J_{0}\left(2 \pi q^{\prime} r\right) r d r d \theta d z
$$

where $J_{0}$ is the zeroth-order Bessel function. This relationship states that the FRT of the diffusion signal gives the radial projection of the PDF, except that instead of the projection being along an infinitely thin line the projection is along a Bessel beam with a width defined by the width of the zeroth-order Bessel function is concentrated 
at the origin. QBI reconstruction has a number of advantages including linearity in the signal, model-independence, and the ability to resolve IVOH. In regions containing intravoxel fiber curvature, QBI arguably provides a more accurate representation of the number of fiber populations present [122].

Descouteaux et al. [123] were also Calculating the ODF by modeling the signal with high order SH series using a Laplace Beltrami regularization method developed for the ADC profile estimation, which lead to an elegant mathematical simplification of the Funk Radon transform which approximates the ODF. The algorithm obtained was fast for the extraction of a robust regularized model independent ODF approximation at each voxel of the raw HARDI data, which offers advantages. this Algorithm will be explained in details in the methods section (6.4).

\subsection{Anisotropy Measurements}

The anisotropy measurement is yet an important parameter to be calculated from the tensors, as FA, RA and VR for single tensor, GA for higher order tensors. So corresponding anisotropy measures, calculated the form the ODF, will be introduced.

Frank et.al [38] proposed the idea of using spherical harmonic decomposition (SHD) to characterize the 3-D apparent diffusion coefficient (ADC) profile measured by HARD imaging. In general, the lower order (0th or 2nd) spherical harmonics (SH) obtained by SHD represent the isotropic diffusion or single fiber diffusion patterns,

whereas the higher orders (4th or higher) represent non-Gaussian patterns associated with intravoxel multiple fiber components. However, compared with DTI, a major disadvantage of the SHD method is that the calculated SHs are actually rotationvariant, i.e., the magnitude and the phase value of the decomposed SH (1st order or higher) change with the rotation of the diffusion profile with respect to the coordi- 
nate system. In the same paper, the fractional multifiber index (FMI) is introduce to measure the significance of a multiple-fiber channel is the fractional even order greater than 0 in that channel.

$$
F M I=\frac{\sum_{L \geq 4} \sum_{M}\left|A_{L, M}\right|^{2}}{\sum_{M}\left|A_{L=2, M}\right|^{2}}, \mathbf{L} \text { even }
$$

Chen et al. [124] calculated the isotropicity of a voxel using:

$$
\begin{aligned}
R_{0} & =\frac{\left|A_{0,0}\right|}{\sum_{l=0,2,4} \sum m=-l^{l}\left|A_{l, m}\right|} \\
G A & =\frac{\sum m=-2^{m=2}\left|A_{2, m}\right|}{\sum_{l=0,2,4} \sum m=-l^{l}\left|A_{l, m}\right|}
\end{aligned}
$$

Large values of $R_{0}$ and $G A$ correspond to isotropic and one-fiber diffusion, respectively. For the rest of points, the number of local maxima of ADC, together with the weights of the variances at the local maxima were used to classify voxels as isotropic, one-fiber or two-fiber diffusion. This procedure is more precise, but there are many measures involved and thus more thresholds needed to be set subjectively [124].

The same group introduced another anisotropy measure based on cumulative residual entropy (CRE). [125] CRE is a measure of uncertainty/information in a random variable. Let $X$ be a random variable in $R, \mathrm{CRE}$ of $\mathrm{X}$ is defined by

$$
C R E(X)=-\int_{R_{+}} P(X>\lambda) \log (P(X>\lambda)) d \lambda
$$

where $R_{+}=X \in R \mid X \geq 0$. The authors used $C R E\left(e^{-b d}\right)$ rather than $d$ to characterize diffusion anisotropy, where $d$ is recovered from HARD measurements. $e^{-b d}$ was chosen for the following reasons:

- the convergence of the magnitude of $e^{-b d}$ is in the order of $10^{-1}$, which is larger than that of ADC itself.

- $e^{-b d}$ is a smooth approximation of the data $\frac{S}{S_{0}}$ 


$$
C R E\left(e^{-b d}\right)=-\sum_{i=2}^{M} P\left(e^{-b d}>\lambda_{i}\right) P\left(e^{-b d}>\lambda_{i}\right) \Delta \lambda_{i}
$$

where $\lambda_{1}<\lambda_{2}<\ldots<\lambda_{M}$ is range of $e^{-b d}$ at voxel $\Delta \lambda_{i}=\lambda_{i}-\lambda_{i-1}$ is the absolute

difference between two adjacent $e^{-b d}$. In most of the cases, the variation of $e^{-b d}$ is the largest for one-fiber diffusion voxels, smaller for two-fiber diffusion and smallest for isotropic voxels. This also explains why CRE is the largest for one-fiber, medium for two-fiber and smallest for isotropic diffusion voxels [125].

$R_{0}$ cannot detect multi-fiber diffusion as it measures the significance of the second order components in SHS. Nonsignificant difference between $R_{2}$ and $F A$ is observed. But CRE differs much from R2 and FA. Furthermore, the smallness of magnitude of $R_{2}$ or $F A$ is unable to distinguish between isotropic and two-fiber diffusion, while CRE does better job [125]. The main problem in all these measures is that they are not rotationally invariant, although the CRE was the least variant between them.

\subsection{Methods}

In this section we will be introducing the ODF methodology developed by Descouteaux et. al [123] to calculate ODF, which will be used in the data analysis . The authors' solution is based on modeling the signal with high order SH series using a LaplaceBeltrami regularization method developed for the ADC profile estimation. This leads to an elegant mathematical simplification of the FunkRadon transform (FRT) which which is used to approximate the ODF.

\subsubsection{Spherical Harmonics}

The spherical harmonics are the angular portion of a set of solutions to Laplace's equation. Represented in a system of spherical coordinates, Laplace's spherical harmonics 
are a specific set of spherical harmonics that forms an orthogonal system, first introduced by Pierre Simon de Laplace. Spherical harmonics are important in many theoretical and practical applications, particularly in the computation of atomic orbital electron configurations [126], representation of gravitational fields [127], geoids [128], and the magnetic fields of planetary bodies and stars [129], and characterization of the cosmic microwave background radiation [130]. In this section spherical harmonics and the Legendre function will be described.

\subsubsection{Legendre Function}

The first class of orthogonal functions is named after Adrien-Marie Legendre [131]. In general represented by the symbol $P_{m}^{l}$, the associated Legendre polynomials are real-valued and defined over the range $[-1,1]$. An explicit definition is

$$
P_{l}^{m}=\frac{(-1)^{m}}{2^{l} l !} \sqrt{\left(1-x^{2}\right)^{m}} \frac{\partial^{l+m}}{\partial x^{l+m}}\left(x^{2}-1\right)^{l}
$$

Although it is rarely used for computational purposes, because the evaluation is tricky and numerically unstable. The function takes two integer arguments $l$ and $m$ which are constrained by $l \in N_{0}$ and $m \in[0 ; l], l$ is used as the band index to divide the class into bands of functions resulting in a total of $(l+1) l$ polynomials for a $l^{\text {th }}$ band series. With respect to $l$, the associated Legendre polynomials obey the orthogonality relationship.

However, for different $m$ on the same band, the polynomials are orthogonal with respect to a different constant and another weighting function. If neither $m=m_{0}$ nor $l=l_{0}$ the polynomials are not orthogonal at all. When used in spherical harmonics, this orthogonality needs to be established by another orthogonal polynomial.

The associated Legendre polynomials 6.1 can also be defined using a set of recurrence relations

$$
P_{m}^{m}(x)=(-1)^{m}(2 m-1) !\left(1-x^{2}\right)^{m / 2}
$$




\begin{tabular}{|c|c|c|}
\hline 1 & $\mathbf{m}$ & $\mathbf{P}_{1}^{\mathbf{m}}(\mathbf{x})$ \\
\hline 0 & 0 & 1 \\
\hline 1 & 0 & $x$ \\
\hline 1 & 1 & $-\sqrt{1-x^{2}}$ \\
\hline 2 & 0 & $\frac{1}{2}\left(3 x^{2}-1\right)$ \\
\hline 2 & 1 & $-3 x \sqrt{1-x^{2}}$ \\
\hline 2 & 2 & $3\left(1-x^{2}\right)$ \\
\hline 3 & 0 & $\frac{1}{2}\left(5 x^{3}-3 x\right)$ \\
\hline 3 & 1 & $\frac{3}{2}\left(1-5 x^{2}\right) \sqrt{1-x^{2}}$ \\
\hline 3 & 2 & $15 x\left(1-x^{2}\right)$ \\
\hline 3 & 3 & $-15 \sqrt{\left(1-x^{2}\right)^{3}}$ \\
\hline
\end{tabular}

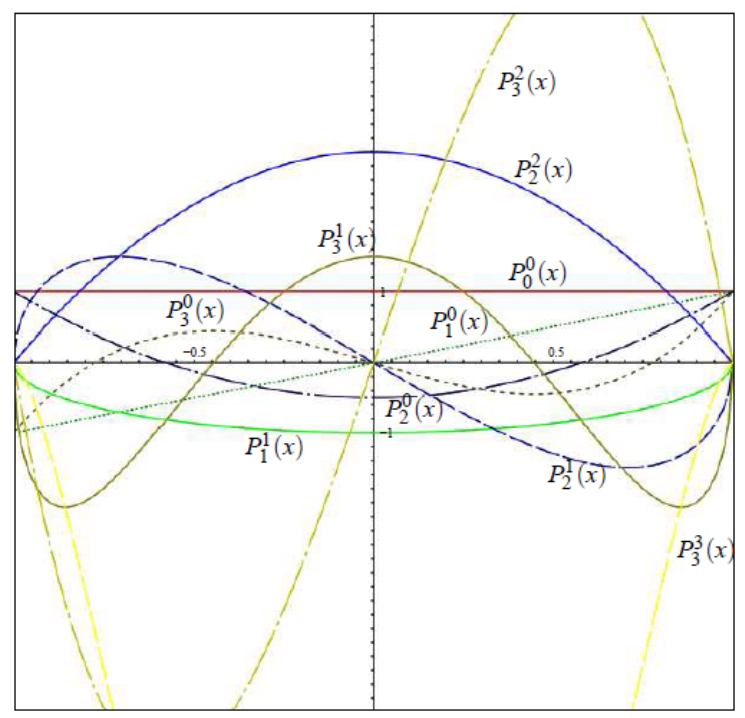

Figure 6.1 First Four bands $1=0, \ldots, 3$ of the associated Legendre Polynomials

$$
\begin{gathered}
P_{m+1}^{m}(x)=x(2 m+1) P_{m}^{m}(x) \\
(l-m) P_{l}^{m}(x)=x(2 l-1) P_{l-1}^{m}(x)-(l=m-1) P_{l-2}^{m}(x)
\end{gathered}
$$

which will come in handy when implementing the function in a computer application, especially since they are easier to compute and less susceptible to numerical errors compared to other methods. To evaluate a given function value $P_{m}^{l}(x)$ primarily equation 6.21 is used to generate the highest $P_{m}^{m}$ possible. Thereafter for $l=m$ the correct value has been computed. Otherwise all that is left to do is to raise the band, so equation 6.22 is used once to get to the next band, and then equation 6.23 can be iterated (because it depends on $l-1$ and $l-2$ results the second rule needs to be applied once) until the correct answer is found.

\subsubsection{Spherical Harmonics (SH)}

The associated Legendre polynomials can be used to express any piecewise continuous function over the interval $[-1,1]$ either as an infinite series of polynomials, a finite series of polynomials for a band-limited approximation or a finite series of polynomials 
in case the function itself does not have frequencies higher than a certain threshold. When looking at the definition of spherical coordinates $(\theta, \phi)$ :

$$
s=(x, y, z)=(\sin (\theta) \cos (\phi), \sin (\theta) \sin (\phi), \cos (\theta))
$$

where s are locations on the unit sphere.

Spherical Harmonics define an orthonormal basis over the sphere. The basis functions are defined as

$$
Y_{l}^{m}(\theta, \phi)=N_{l}^{m} P_{l}^{|m|}(\cos (\theta)), l \epsilon N,-l \leq m \leq l
$$

Where $N_{l}^{m}$ are the normalization constants. The normalization factor can then be derived from

$$
\int_{S} Y_{l}^{m}(\omega) \overline{Y_{l^{\prime}}^{m^{\prime}}} \sin (\theta) d \omega=\delta_{m m^{\prime}} \delta_{l l^{\prime}}
$$

which concurrently proves the orthogonality of the spherical harmonics. The $\sin (\theta)$ weights the function values by the distance from the equator. This is due to the fact that integrating spherical coordinates can be seen as integrating small patches on the sphere. Solving Equation 6.26 by expanding $Y_{l}^{m}$ yields:

$$
\begin{array}{r}
\int_{0}^{2 \pi} \int_{0}^{\pi} Y_{l}^{m}(\theta, \phi) \overline{Y_{l}^{m^{\prime}}}(\theta, \phi) \sin (\theta) d \theta d \phi, \\
=\int_{0}^{2 \pi} \int_{-1}^{1} Y_{l}^{m}(\theta, \phi) \overline{Y_{l}^{m^{\prime}}}(\theta, \phi) d(\cos (\theta)) d \phi, \\
=\int_{0}^{2 \pi} \int_{-1}^{1} N_{l}^{m} N_{l^{\prime}}^{m^{\prime}} P_{l}^{m}(\cos (\theta)) N_{l^{\prime}}^{m^{\prime}}(\cos (\theta)) e^{i m \phi} e^{i \bar{m} \phi} d(\cos (\theta)) d \phi, \\
=N_{l}^{m} N_{l^{\prime}}^{m^{\prime}} \int_{-1}^{1} P_{l}^{m}(\cos (\theta)) N_{l^{\prime}}^{m^{\prime}}(\cos (\theta)) d(\cos (\theta)) \int_{0}^{2 \pi} e^{i m \phi} e^{i \bar{m} \phi} d \phi
\end{array}
$$

After solving this integral

$$
N_{l}^{m} N_{l^{\prime}}^{m^{\prime}} \frac{4 \pi}{2 l+1} \frac{(l+m) !}{(l-m) !} \delta_{l l^{\prime}} \delta_{m m^{\prime}}=\delta_{l l^{\prime}} \delta_{m m^{\prime}}
$$

assuming that $m=m^{\prime}$,it becomes obvious that 


$$
N_{l}^{m}=\sqrt{\frac{2 l+1}{4 \pi} \frac{(l-m) !}{(l+m) !}}
$$

Most applications of spherical harmonics require only real valued spherical functions, it is convenient to define the real-valued spherical harmonics function as

$$
Y_{l}^{m}= \begin{cases}\sqrt{2} R\left(Y_{l}^{m}\right)=\sqrt{2} N_{l}^{m} \cos (m \phi) P_{l}^{m}(\cos (\theta)) & \text { if } m>0 \\ Y_{l}^{0}=N_{l}^{0} P_{l}^{0}(\cos (\theta)) & \text { if } m=0 \\ \sqrt{2} I\left(Y_{l}^{m}\right)=\sqrt{2} N_{l}^{|m|} \sin (|m| \phi) P_{l}^{|m|}(\cos (\theta)) & \text { if } m<0\end{cases}
$$

While the complex spherical harmonic basis includes a pair of sines, the separated imaginary and real parts of the real spherical harmonics only have one sine, and thus the normalization needs to be adjusted by a factor of $\sqrt{2}$ for those cases.

\subsubsection{ODF Calculation}

The SH basis is designed to be symmetric, real and orthonormal. Symmetry is ensured in the ODF by choosing only even order $\mathrm{SH}$ and the ratios in front of each term also ensure that the modified basis is real and orthonormal.

The signal at each of the $\mathrm{N}$ gradient directions $\mathrm{i}$ is approximated as

$$
S\left(\theta_{i}, \phi_{i}\right)=\sum_{j=1}^{R} c_{j} Y_{j}\left(\theta_{i}, \phi_{i}\right)
$$

where $R=(l+1)(l+2) / 2$ is the number of terms in the modified SH basis $\mathbf{Y}$ of order l. Letting $\mathbf{S}$ be the $N \times 1$ vector representing the input signal for every encoding gradient direction, $\mathbf{C}$ the $R \times 1$ vector of $\mathrm{SH}$ coefficients $c_{j}$ and $\mathbf{B}$ is the $N \times R$ matrix constructed with the discrete modified SH basis

$$
B=\left(\begin{array}{cccc}
Y_{1}\left(\theta_{1}, \phi_{1}\right) & Y_{2}\left(\theta_{1}, \phi_{1}\right) & \cdots & Y_{R}\left(\theta_{1}, \phi_{1}\right) \\
\vdots & \vdots & \ddots & \vdots \\
Y_{1}\left(\theta_{N}, \phi_{N}\right) & Y_{2}\left(\theta_{N}, \phi_{N}\right) & \cdots & Y_{R}\left(\theta_{N}, \phi_{N}\right)
\end{array}\right)
$$


The set of equations can be rewritten as an over-determined linear system $\mathbf{S}=\mathbf{B C}$, by solving for the SH series coefficients $c_{j}$, where $c_{j}=\int_{\Omega} S(\theta, \phi) Y_{i}(\theta, \phi) d \Omega$.

At this point, a local regularization can be directly used into the fitting procedure. This is to be able to use a high order estimation without overmodeling, the small perturbations because of noise in the input diffusion MRI signal. Thus, a measure, $\mathrm{E}$, of the deviation from smoothness of a function $f$ is defined on the unit sphere, as $E(f)=\int_{\Omega}\left(\Delta_{b} f\right)^{2} d \Omega$, where $\Delta_{b}$ is the Laplace Beltrami operator. The Laplace Beltrami operator is a natural measure of smoothness for functions defined on the unit sphere. If the spherical function $f$ is parameterized with $\mathrm{SH}$, the Laplace Beltrami operator is very simple to evaluate when acting on this parametrization as it satisfies the relation $\Delta_{b} Y_{l}^{m}=-l(l+1) Y-l^{m}$. This relation also holds for the modified SH basis Y. Using the orthonormality of the modified SH basis, the above functional E can be rewritten as:

$$
E(f)=\int_{\Omega} \Delta_{b}\left(\sum_{p} c_{p} Y_{p}\right) \Delta_{b}\left(\sum_{q} c_{q} Y_{q}\right) d \Omega=\sum_{j=1}^{R} c_{j}^{2} l_{j}^{2}\left(l_{j}+1\right)^{2}=\mathbf{C}^{\mathbf{T}} \mathbf{L} \mathbf{C}
$$

where $\mathbf{L}$ is simply the $R \times R$ matrix with entries $l_{j}^{2}\left(l_{j}+1\right)^{2}$ along the diagonal $l_{j}$ is the order associated with the $j^{\text {th }}$ coefficient. Therefore, the quantity to be minimized can be expressed in matrix form as:

$$
M(C)=(S-B C)^{T}(S-B C)+\lambda C^{T} L C
$$

where $\lambda$ is the weight on the regularization term. The coefficient vector minimizing this expression can then be determined just as in the standard least-squares fit $(\lambda=$ 0 ). Then, the generalized expression for the desired SH series coefficient vector.

$$
C=\left(B^{T} B+\lambda L\right)^{-1} B^{T} S
$$

From this SH coefficient vector, the signal on the q-ball can be recovered for any $(\theta, \phi))$ using the equation (6.31). 
Intuitively, this approach penalizes an approximation function for having higher order terms in its modified SH series, which eliminates most of the high order terms due to noise while leaving those that are necessary to describe the underlying function. However, obtaining this balance depends on choosing a good value for the parameter $\lambda$.

\subsubsection{Orientation Distribution Function Anisotropy Measure [ODFA]}

Kim [132] introduced an anisotropy parameter, to establish links between geometrical features and mechanical performance of nonwoven fabrics and the point bonded (spot bonded) nonwoven using ODF, which represents composite materials anisotropy of fiber orientation distribution with respect to the preferred fiber orientation in order to check the alignment of this preferred fiber to a referred direction.

$$
<\cos ^{2}(\theta)>=\frac{\int_{0}^{\pi} \cos ^{2}\left(\theta-\theta_{\text {ref }}\right) \psi(\theta) d \theta}{\int_{0}^{\pi} \psi(\theta) d \theta}
$$

where $\theta$ : orientation angle, $\theta_{\text {ref }}$ : angle at a referred direction, $\psi(\theta)$ : ODF frequency at each orientation angle.

This parameter's range varies between 0 and 1, 1 indicates perfect alignment of the fibers parallel to the reference direction and 0 indicates perfect perpendicular alignment. The advantage of this isotropicity measure that it is rotationally invariant. This function is actually representing the convolution of the ODF function with the $\cos ^{2}$ function, It can be extended to any cos function of any even power, which technically means more anisotropic convolution function.

This inspired us to explore its use in ODF DTI analysis, can be extended to be calculated for the ODF function overall the surface of the sphere as the Isotropic 
Measure (IM):

$$
\begin{gathered}
I M=\frac{1}{\pi^{2}} \int_{0}^{\pi} \int_{0}^{\pi} \frac{\left.\int_{0}^{\pi} \int_{0}^{\pi} d o t^{2}\left(v(\theta, \phi), v\left(\theta_{r e f}, \phi_{r e f}\right)\right) \psi(\theta, \phi)\right) d \theta_{\text {ref }} d \phi_{\text {ref }}}{\int_{0}^{\pi} \int_{0}^{\pi} \psi(\theta, \phi) d \theta d \phi} d \theta d \phi \\
I M=\frac{1}{\pi^{2}} \sum_{0}^{\pi} \sum_{0}^{\pi} \frac{\left.\sum_{0}^{\pi} \sum_{0}^{\pi} d_{o t^{2}}\left(v(\theta, \phi), v\left(\theta_{r e f}, \phi_{r e f}\right)\right) \psi(\theta, \phi)\right)}{\sum_{0}^{\pi} \sum_{0}^{\pi} \psi(\theta, \phi)}
\end{gathered}
$$

If the material is totally isotropic, the Anisotropy the ODF (ODFA) will be 1 and decrease as the anisotropy increases. Since the most of the anisotropic measures are getting larger as the anisotropy increases. and the IM is not a linear function as the anisotropicity increases, we used the ODFA measure as a function of this IM as follows:

$$
O D F A=1-\sqrt{I M}
$$

The new anisotropy measure performance was compared to the CRE and $R_{0}$ to prove the rotation invariance property as a function of the separation angle between 2 tensors. Mathematica was used to find a closed form of the ODFA, to decrease the processing time. the new measure was also compared to other anisotropy measures to demonstrate the linearity, rotation invariance and noise robustness.

\subsubsection{Monte Carlo Simulation}

Monte Carlo Simulations were performed to assess the effect of noise on the anisotropy indices estimated from noisy attenuation signals through the calculation of the apparent diffusion coefficient and spherical Harmonics coefficients. Diffusion tensors whose eigen values are representative of human brain tissues anisotropy, white matter (WM), gray matter (GM) and cerebro-spinal fluid (CSF) were used. For simplicity , the anisotropic tissues principal axes coincided with the laboratory frame of reference was assumed. Noise free Attenuation was calculated form these tensors using Equation (2.11). Rician thermal noise in the MR measurement was simulated by 
generating complex random numbers whose real and imaginary parts were Gaussian distributed with zero mean and standard deviation scales to the signal to noise ratio (SNR) to represent the Rician noise distribution defined by $[133,134]$. The noise free attenuation is added to the real component of the complex noise, and the magnitude of the complex number to generate the noisy signal. The rank-2 tensor, and the SH coefficients and their corresponding anisotropy measure FA and ODFA respectively were calculated. The previous steps were repeated 1000 times, the mean and the standard deviation of these anisotropy measurements were calculated to measure the validity of these measurements.

\subsection{Results and Discussion}

Tensors with different anisotropcity were used to calculate the their corresponding ODFA, FA and GA, to compare the relation between such measures. The attenuation of a single tensor of different anisotropy values was used to calculate the corresponding FA for rank-2 tensor, GA for rank-4 tensors IM and ODFA for the SH ODF coefficients. As shown in Figure [6.2], the IM is following a nonlinear function with respect to the FA. The relation between the ODFA and the FA is near linear with almost the same slope in most of the range except for very high isotropic materials. GA, and FA function is nonlinear. the linearity of the ODFA can be considered as one of the advantages of the new measure. Some of the most popularly anisotropy measurements, CRE, Variance and $R_{2}$ which are not tensor based parameters, characterizing diffusion anisotropy, were used for rotation invariance comparison. In Figure[6.3]. The total attenuation, corresponding to two tensors with eigen values $\left[8 \times 10^{-2}, 10^{-2}, 3 \times 10^{-2}\right]$ where eigen vector corresponding to the major eigen values, is used to calculate the corresponding effective anisotropy. The synthetic data is constructed as follows: by 


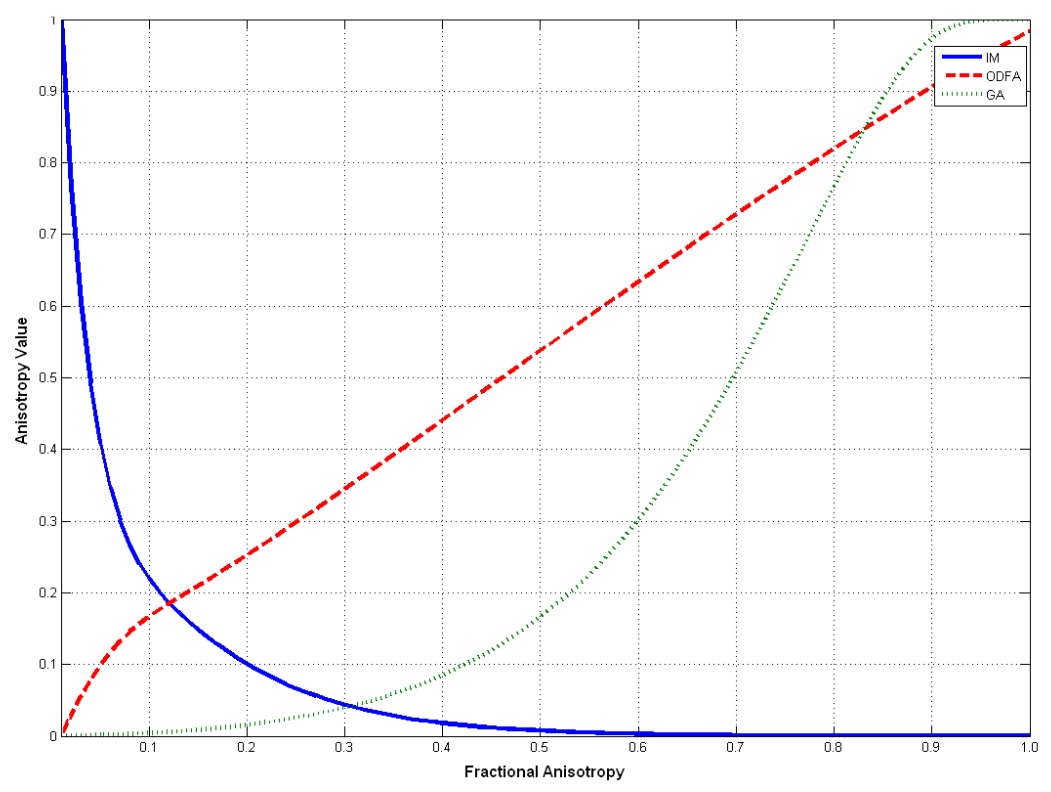

Figure 6.2 GA and ODFA values corresponding to FA

setting $D_{1}$ and $D_{2}$ with eigen values as defined previously. The first tensor $D_{1}$ has a fixed direction and the second tensor $D_{2}(\psi)$, where $\psi \in[0, \pi]$. ODFA, CRE, variance and $R_{2}$ were calculated for each separation angle, and then each curve is normalized to 1 . The results proves the rotational Invariance of the new measure. Since the processing time for one ODFA calculation knowing the corresponding SH ODF coefficient takes $0.11 \mathrm{sec}$ on a 2.1 Dual core Intel processor and 4.00 GB DRAM machine because of the four nested summation functions. The processing time will be taking hours when it is used to calculate the anisotropy of a 3D volume. We found that it would be more convenient to find a closed form to decrease this processing time to less than $0.1 \mathrm{~m}$ sec. Mathematica was used to generate a closed form for this anisotropy measurement. the ODFA for SH of order 6 can be calculated using the following equation: 


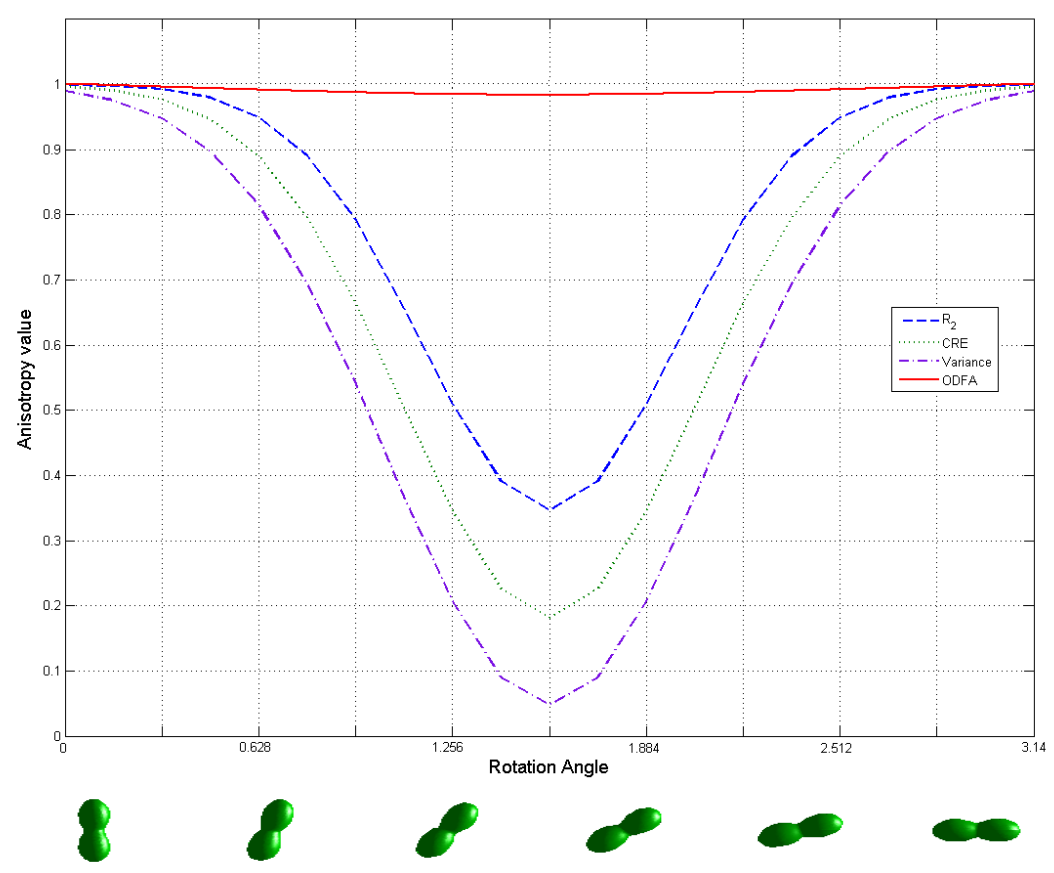

Figure 6.3 Rotation variance of the closed form of the ODFA for different Anisotropy values

and for order 8

$$
I M=\frac{3}{8} \times \frac{\left.A_{0} \times c(0,0)+A_{1} \times c(2,2)\right)+A_{2} \times c(4,4)+A_{3} \times c(6,6)+A_{4} \times c(8,8)}{\left.B_{0} \times c(0,0)+B_{1} \times c(2,2)\right)+B_{2} \times c(4,4)+B_{3} \times c(6,6)+B_{4} \times c(8,8)}
$$

where $A_{0}=16384, A_{1}=6144 \sqrt{5}, A_{2}=9472, A_{4}=2160 \sqrt{13}, A_{5}=1645 \sqrt{17}, B_{1}=$ $16384, B_{2}=4096 \sqrt{5}, B_{3}=6912, B_{4}=1600 \sqrt{13}, B_{5}=1225 \sqrt{17}$. This can be then generalized to any SH order, so this ODFA formulation will be used for further analysis to decrease the processing time. To check rotational invariance of the new formulation for the ODFA. A single tensor with different anisotropy values and different directions has been used, the corresponding ODFA is calculated, mean, minimum and maximum value of the anisotropy were plotted in figure [6.4]. the calculated ODFA is near rotationally invariant, the variance increases with the increase of the anisotropy. the maximum rotation variance is, which can be neglected. this variance is due to the 


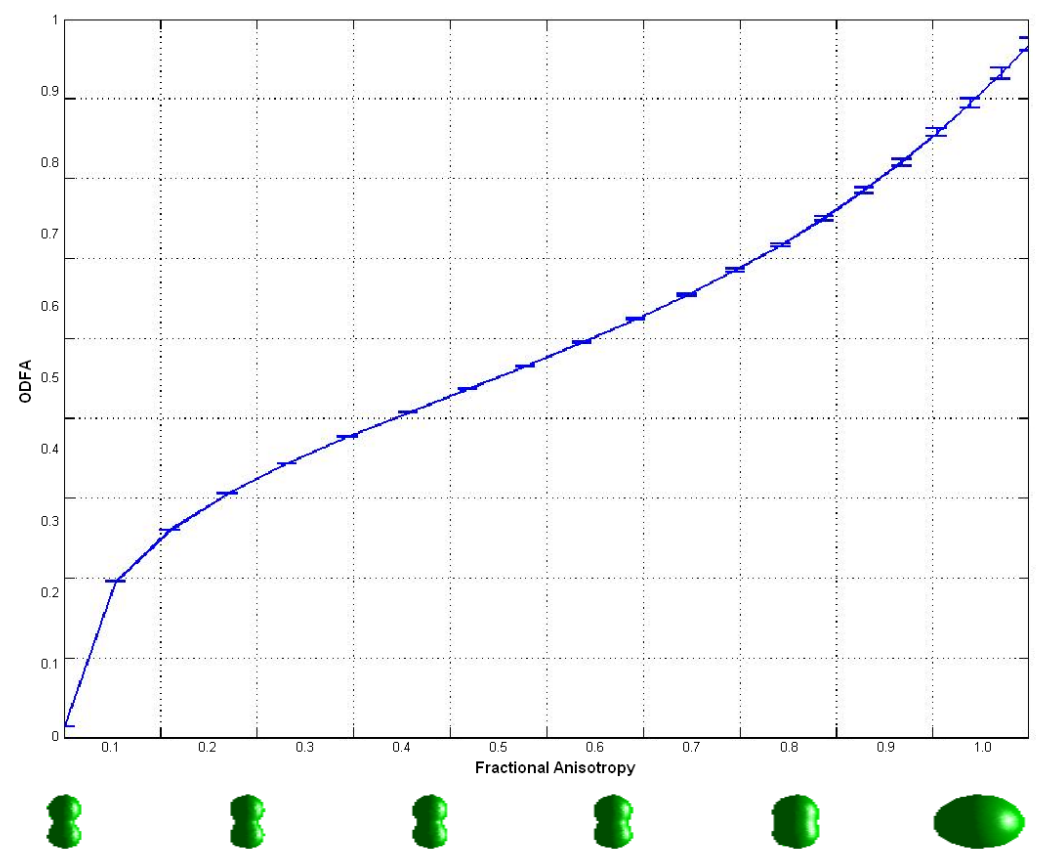

Figure 6.4 Rotation variance of the closed form of the ODFA for different Anisotropy values

approximation of the four nested summation function and the spherical harmonics function to the ODF coefficient.

Linearity and rotation invariance of the new ODFA closed form function are further investigated as a function of change of anisotropy the different anisotropic tensor is shown in figure [6.4].

Figure (6.5) is studying how will be the ODFA of white matter's tensor, having eigen values $\left[0.3 \times 10^{-2}, 0.9 \times 10^{-2}, 1.7 \times 10^{-2}\right]$, changes with the orientation changing from $[0, \pi]$. Monte Carlo simulation is used to study the behavior of the anisotropy measure due to noise with different signal to noise ratio (SNR) for the 3 different brain tissues: White Matter (WM), Gray Matter (GM) and Cerebrospinal fluid (CSF). the eigen values used to represent these three materials are $[0.3 \times$ $\left.10^{-2}, 0.9 \times 10^{-2}, 1.7 \times 10^{-2}\right]$ for $\mathrm{WM},\left[0.8 \times 10^{-2}, 0.9 \times 10^{-2}, 1.1 \times 10^{-2}\right]$ for GM and $\left[0.208 \times 10^{-2}, 0.216 \times 10^{-2}, 0.217 \times 10^{-2}\right]$ for CSF. The mean and variance of 
anisotropy for both the ODFA and the FA with different SNR of the 3 materials are shown in Figures $[6.5,6.6,6.7]$. As a general observation we can see that the ODFA is stabilizing at lower SNR than that for the FA, and even the deviation of the ODFA from the true value is less in case of the ODFA in case of low SNR, which means that the ODFA is much more robust for the noise than the ODFA. At high SNR, the anisotropy calculation is trivial, but otherwise, noise is affecting the attenuation, which is consequently affecting the estimation of the tensor and the spherical harmonic coefficient,and consequently propagated through in the anisotropy derived quantities [135]. The matrix perturbation theory analysis shows that if the perturbation order $\epsilon$ in a matrix A, then an eigen value $\lambda$ may be perturbed by an amount $\epsilon / s(\lambda)$. Thus, if $s(\lambda)$ is small, then $\lambda$ is appropriately regarded as ill-conditioned. A small $s(\lambda)$ implies that $\mathrm{A}$ is near a matrix having a multiple eigen-value. In particular, if $\lambda$ is distinct and $s(\lambda)<1$, then there exists an $E$ such that $\lambda$ is repeated eigenvalue of $A+E[136]$

$$
\frac{\|E\|_{2}}{\|A\|_{2}} \leq \frac{s(\lambda)}{\sqrt{1-s(\lambda)^{2}}}
$$

In case of tensor estimation the noise perturbs all the indices calculation, which in its turn perturbs the eigen values, making misclassification more likely. It overestimates the highest eigenvalues, and underestimates the lower eigen values. This bias enhances artificially the mean anisotropy and introduces a sorting bias. Negative eigenvalues are detected for higher SNR preventing an interpretation of the tensor as a quantity describing diffusion. That makes the estimation of the tensor is less robust. For linear regression model, the diagonal elements are underestimated [137,138]. Since such perturbation doesn't affect the SH ODF estimation, this leads to noise robustness of the ODFA.

The FA, GA and ODFA were calculated for a single human brain HARDI MRI data. The GA appears to be much more blurred or less contrast image which highlight 


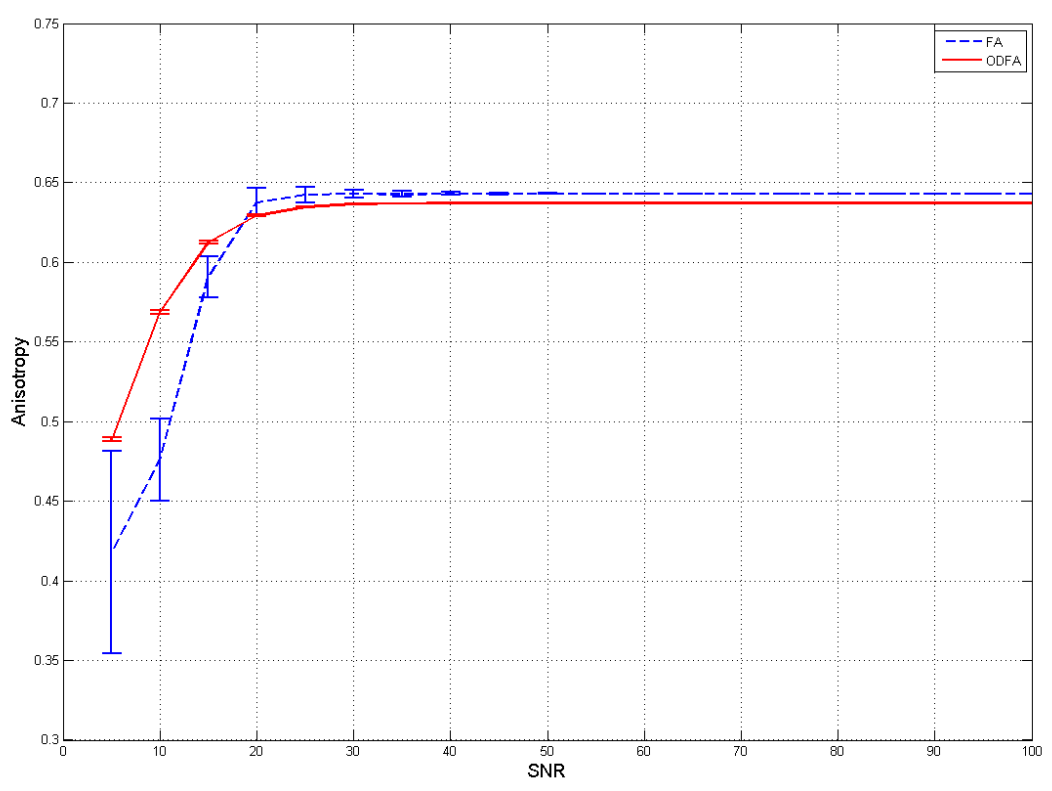

Figure 6.5 Effect of noise on the FA and ODFA for White Matter tissue

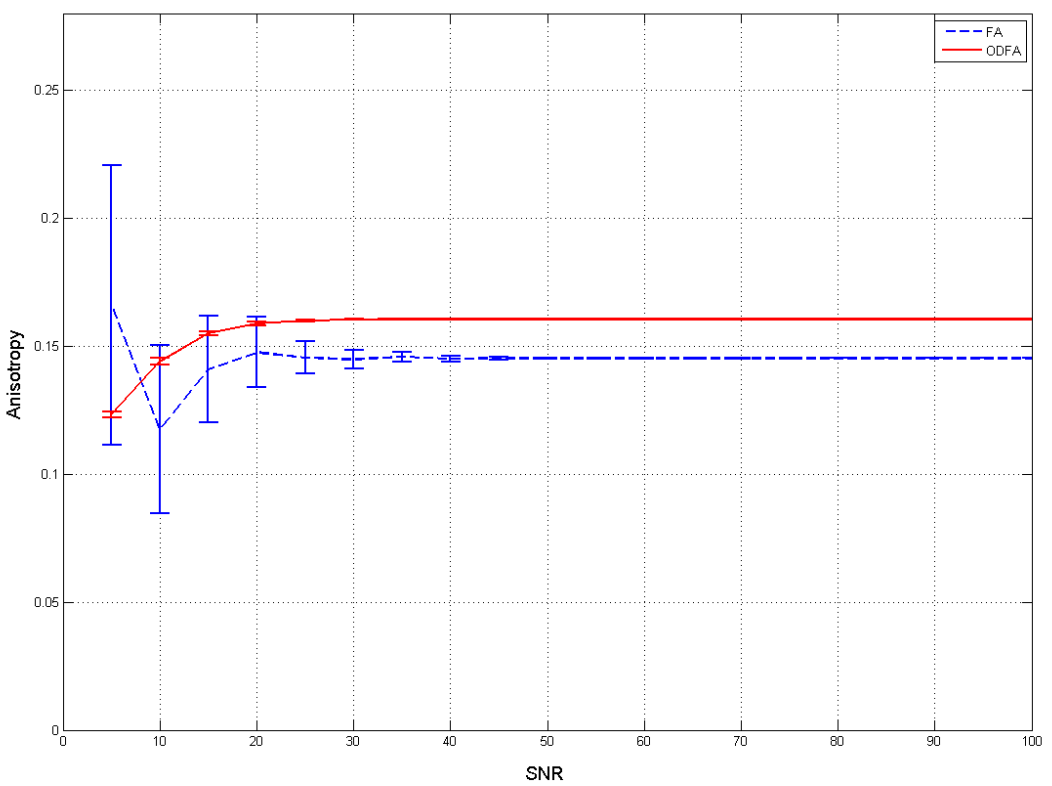

Figure 6.6 Effect of noise on the FA and ODFA for Gray Matter tissue 


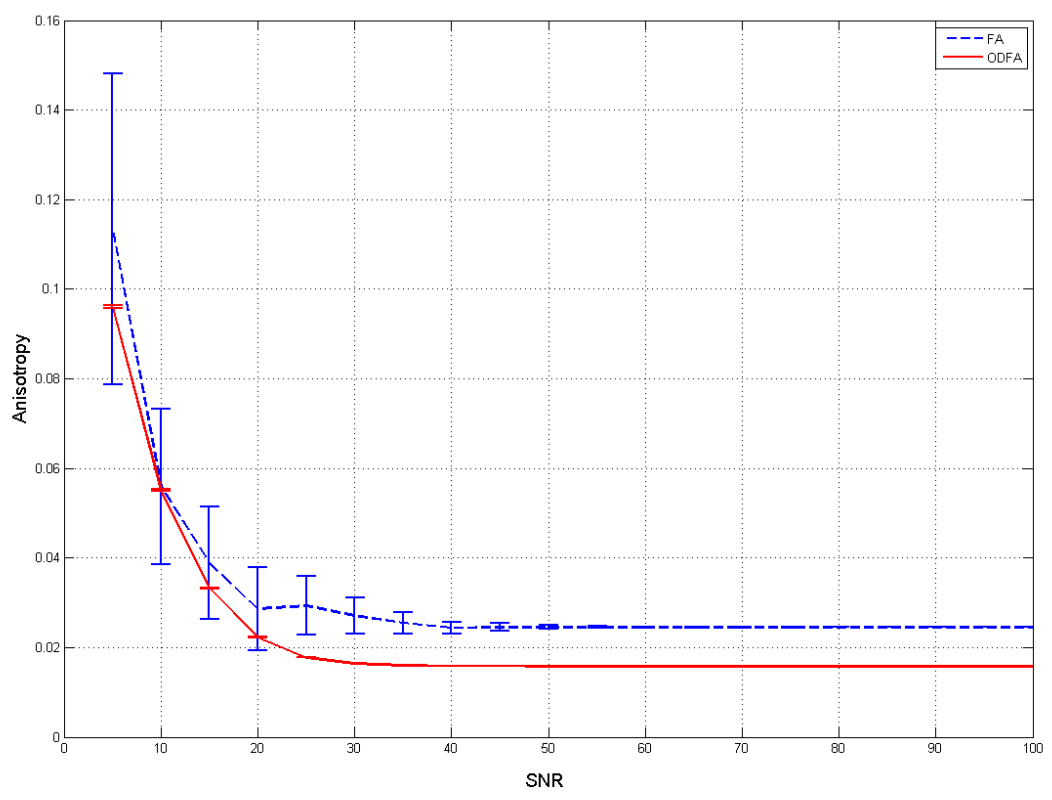

Figure 6.7 Effect of noise on the FA and ODFA for Cerebrospinal Fluid tissue

in another way that there are some anisotropicity occurring, which is most apparent in the ODFA measurement for the peripheral temporal lobes and the basal ganglia, which correspond to have multi fibers in these regions. 


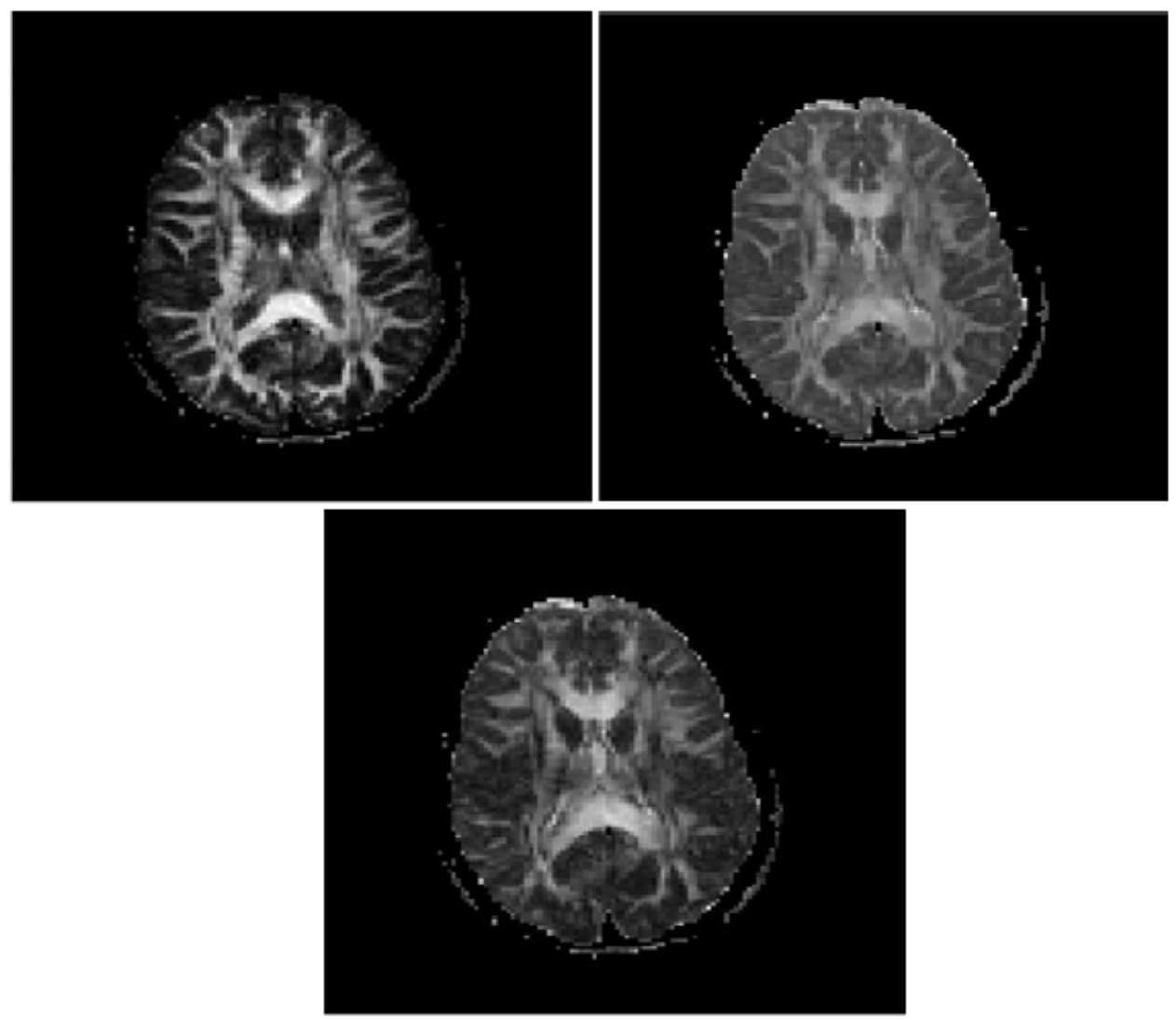

Figure 6.8 Anisotropy measurements for an axial human brain slice: Top [FA (left), GA (right)], Bottom [ODFA] 


\section{Chapter 7}

\section{Conclusion and Future Work}

\subsection{Conclusions}

A new scheme for tensor field interpolation which can be extended to tensors of any arbitrary rank, has been presented. This scheme can be used for interpolation or even approximation. It is physically-based on mass conservation and fiber smoothness is guaranteed. The method is computationally efficient - It requires only a sparse matrix-vector multiplication at each step, and the matrix can be precomputed since it is independent of the data. Results show that the technique better preserves FA in case of rank 2 and GA in case of higher rank tensors during interpolation in some cases than linear and log-Euclidean interpolation. Results for FACT technique, used for shape geometry analysis, showed that the tract of the subdivided model had the least error than that the linearly interpolated tract and the downsampled tract. Frobenius norm error comparison between the subdivided volumes and the linearly interpolated volumes to the original volume showed error decrease to $10.00 \%$ for the subdivision volume than $25.19 \%$ for the linearly interpolated volume.

Bezier Curve approximation can be used for subdivision in any arbitrary point 
without the need of going the whole subdivision process in case of the regular subdivision. For higher degrees, such as $n=7$ the results approximate those of the Bezier subdivision approach.

The generalized Helmholtz decomposition was also used provide intuitive and useful information about the structure of tensor fields. Based on this decomposition, new scalar measures for DT-MRI can be formulated which convey topological information. Specifically, local peaks in magnitude of divergence and curl correspond to critical lines in the tensor field. The presented formulations are general with respect to tensor rank and do not require eigenvalues to be computed. The decomposition and the new scalar measures are easy to compute and can be used to provide useful structural measurement. The new Scalar measures can be used to develop a more complete topological characterization of high rank tensor fields, including more types of critical points and sparse matrices. To explore the potential field $\phi$ and stream field $\psi$ to see if useful information can be extracted directly from them is a another future problem to be investigated.

A novel rotationally invariant anisotropy measure has been used to calculate the anisotropy from the SH-ODF coefficient ODF and Anisotropy measurement. The algorithm is based on the convolution of $\cos ^{2}$ function with the ODF. the new anisotropy measure has the advantage of being rotationally invariant, the closed form of the ODFA has been calculated and used to check linearity and robustness of the noise. ODFA has an approximate linear relation with the FA. Experiments on the dataset of human brain HARD MRI data showed the effectiveness and robustness of the proposed model in the characterization of diffusion anisotropy. they have less error than the FA in case of low SNR. The results were able to retain more details in multi-crossing fiber areas that appears totally isotropic in case FA measurement as the peripheral temporal lobes and the basal ganglia. This new measure is very 
permissible that solved the problem of anisotropy measure that are based on model assumption.

\subsection{Future Work}

As future work for the subdivision, the tensor basis functions underlying this subdivision scheme need to be investigated. Applications for the use of these splines include tensor field regularization, tractography and model-based tensor field segmentation. More complete topological characterization of high order tensor field. we can also explore the potential field $\phi$ and the stream field $\psi$ for more useful information.

We also need to study how will the ODFA measure constraint affect fiber tracking constraints as one of the most important applications of DTI, specially in the multicrossing fiber. 


\section{Bibliography}

[1] M. L. Wood and F. W. Wehrli, Principles of Magnetic Resonance Imaging (by D.D. Stark, W.G. Bardley, Jr., Mosby CD Online-products, 1956).

[2] C. Westbrook, MRI at a Glance (Blackwell Science Ltd, 2002).

[3] M. A. Bernstein, K. F. King, and X. J. Zhou, Handbook of MRI Pulses (Elsevier Academic Press, 2004).

[4] D. Weishaupt, V. D. Kchli, and B. Marincek, How Does MRI Work? An Introduction to the Physics and Function of Magnetic Resonance Imaging, 2 ed. (Springer-Verlag Berlin Heidelberg, 2006).

[5] S. C. Bushong, Magnetic Resonance Imaging: Physical and Biological Principles (Mosby-Year Book, Inc., 1996).

[6] G. Liney, MRI from A to Z: A Definitive Guide for Medical Professionals (Cambridge University Press, 2005).

[7] P. J. Basser, "New Histological and Physiological Stains Derived from Diffusion-Tensor MR Images," Annals New York Acad. Sci. 820, 123-138 (1997). 
[8] D. L. Bihan, J. Mangin, C. Poupon, C. A. Clark, S. Pappata, N.Molko, and H. Chabriat, "Diffusion Tensor Imaging: Concepts and Applications," J. Mag. Reson. Imaging 13, 534-546 (2001).

[9] H. Y. Carr and E. M. Purcell, "Effects of Diffusion on Free Precession in Nuclear Magnetic Resonance Experiments," phys. Rev. 94, 630-638 (1954).

[10] E. O. Stejskal and J. Tanner, "Spin Diffusion Measurements: Spin Echoes in the Presence of Time-Dependent Field Gradient," J. Chem. Phys. 42, 288-292 (1965).

[11] R. Brown, "A Brief Account of Microscopical Observations Made in the Months of June, July, and August 1827 on the Particles Contained in the Pollen of Plants; and on the General Existence of Active Molecules in Organic and Inorganic Bodies.," Philosoph. Mag. 4, 161 (1828).

[12] A. Einstein, "Uber Die von der Molekularkinetischen Theorie der Warme Geforderte Bewegung von in Ruhenden Fl," ussigkeiten suspendierten Teilchen. Ann. Physik. 4, 549-560 (1905), english translation in Ref. 41).

[13] A. Einstein, "Sue Theorie der Brownschen Bewengung," Ann. Physik 19, 371381 (1906), english translation in Ref. 41).

[14] M. von Smoluchowski, "Zur Kinetischen Theorie der Brownschen Molekularbewegung under Suspensionen," Ann. Phys. 21, 756-780 (1906).

[15] J. Perrin, "Mouvement Brownien et Rtextmidacuteealittextmidacutee Moltextmidacuteeculaire," Annales de chimie et de physiq 18, 4-114 (1909).

[16] F. Bloch, "Nuclear Induction," Phys. Rev. 70, 460-474 (1946). 
[17] F. Bloch, W. W. Hansen, and M. Packard, "Nuclear Induction," Phys. Rev. 69, 127 (1946).

[18] E. M. Purcell, H. C. Torrey, and R. V. Pound, "Resonance Absorption by Nuclear Magnetic Moments in a Solid," Phys. Rev. pp. 69-37 (1946).

[19] E. Hahn, "Spin Echoes," Phys. Rev. 80, 580-594 (1950).

[20] H. Torrey, "Bloch Equations with Diffusion Terms," Phys. Rev. 104, 563-565 (1956).

[21] D. Woessne, "NMR Spin-Echo Self-Diffusion Measurements on Fluids Undergoing Restricted Diffusion," J. Phys. Chem. 67, 1365-1366 (1963).

[22] D. le Bihan, "Diffusion NMR Imaging," Magn. Reson. 7, 1-30 (1991).

[23] J. Matiello, P. J. Basser, and D. leBihan, "Analytical Expressions for the BMatrix in NMR Diffusion Imaging and Spectroscopy," J. Magn. Reson. 108, 131-141 (1994).

[24] P. J. Basser and C. Pierpaout, "Microstructural and Physiological Features of Tissues Elucidated by Quantitative Diffusion Tensor MRL," J. Magn. Reson. 111, 209-219 (1996).

[25] C. Pierpoali, P. Jezzard, P. Basser, A. Barnett, and G. D. Chiro, "Diffusion Tensor Imaging of the Human Brain," Radiology 201, 637-648 (1996).

[26] M. E. Mosely, Y. Cohen, and J. kucharczyk, "Diffusion-Weighted MR Imaging of Anisotropic Water Diffusion in Cat Central Nervous System," Radiology 177, 439-446 (1990). 
[27] P. Douek, R. Turner, J. Perkar, N. J. Patronas, and D. LeBihan, "MR Color Mapping of Myelin Fiber Orientation," J. Comput. Assist. Tomogr. 15, 923929 (1991).

[28] P. V. Glederen, M. D. Vleeschouwe, and D. des Pres, "Water Diffusion and Acute Stroke," Magn. Reson. Med. 31, 154-163 (1994).

[29] C. H. Neuman, "Spin Echo of Spins Diffusing in a Bounded Medium," J. Chem. Phys. 60, 4508-4511 (1974).

[30] C. and P. J. Basser, "Toward a Quantitative Assessment of Diffusion Anisotropy," Magn. Reson. Med. 36, 893-906 (1996).

[31] A. M. Ulug and C. van Zijl, "Orientation-Independent Diffusion Imaging Without Tensor Diagonalization: Anisotropy Definitions Based on Physical Attributes of the Diffusion Ellipsoid," J. Magn. Reson. Imag 9, 804- 813 (1999).

[32] C. Beaulieu, "The Basis of Anisotropic Water Diffusion in the Nervous System- a Technical Review," NMR in Biomed. 15, 435-455 (2002).

[33] C. Baratti, A. S. Barnett, and C. Pierpaoli, "Comparative MR Imaging Study of Brain Maturation in Kittens with T1, T2, and the Trace of the Diffusion Tensor," Radiology 210, 133-142 (1999).

[34] P. J. Basser and D. K. Jones, "Diffusion-Tensor MRI: Theory, Experimental Design and Data Analysis- a Technical Review," NMR Biomed. 15, 456-467 (2002).

[35] M. E. Mortenson, Computer Graphics Handbook: Geometry and Mathematics (Industrial Press Inc., 1990). 
[36] D. S. Tuch, R. M. Weisskoff, J. W. Belliveau, and V. J. Wedeen, "High Angular Resolution Diffusion Imaging of the Human Brain," Proceedings of the 7th Annual Meeting of ISMRM p. 37 (1999).

[37] V. J. Wedeen, T. Reese, D. S. Tuch, M. R. Weigel, J. Dou, R. M. Weiskoff, and D. Chesler, "Mapping Fiber Orientation Spectra in Cerebral White Matter with Fourier Transform Diffusion MRI," Proceedings of the 8th Annual Meeting of ISMRM p. 82 (2000).

[38] L. Frank, "Characterization of Anisotropy in High Angular Resolution Diffusion-Weighted MRI," Mag. Reson. Med. 47 (2002).

[39] P. J. Basser, J. Matiello, and D. le Bihan, "MR Diffusion Tensor Spectroscopy and Imaging," J. biophys 66, 256-267 (1994).

[40] A. L. Alexander, K. M. Hassan, M. Lazar, J. S. Tsuruda, and D. L. Parker, "Analysis of Partial Volume Effects in Diffusion-Tensor MRI," Magn. Reson. in Med. 45, 770-780 (2001).

[41] R. A. D. Graaf, K. P. J. Braun, and K. Nicolay, "Single Shot Diffusion Trace NMR Spectoscopy," Magn. Reson. in Med. 45, 741-748 (2001).

[42] P. J. Basser, J. Mattiello, and D. L. Bihan, "Estimation of the Effective SelfDiffusion Tensor from the NMR Spin Echo," J. Magn. Reson. Ser. 103, 247254 (1994).

[43] D. L. Bihan and P. van Zijl, "From the Diffusion Coefficient to the Diffusion Tensor," NMR Biomed. 15, 431-434 (2002). 
[44] A. Zalesky and A. Fornito, "A DTI-Derived Measure of Cortico-Cortical Connectivity," Medical Imaging, IEEE Transactions Accepted to be published (2009).

[45] E. Ozarlan and T. H. Mareci, "Generalized Diffusion Tensor Imaging and Analytical Relationships between Diffusion Tensor Imaging and High Angular Resolution Diffusion Imaging," Magn. Reson. Med. 50, 955-965 (2003).

[46] E. Ozarlan, B. C. Vemuri, and T. H. Mareci, "Generalized Scalar Measures for Diffusion MRI using Trace, Variance, and Entropy," Magn. Reson. Med. 53, 866-876 (2005).

[47] E. W. Hsu, D. L. Buckley, J. D. Bui, S. J. Blackband, and J. R. Forder, "TwoCompartment Diffusion Tensor MRI of Isolated Perfused Hearts," Magn. Reson. Med. 45, 1039-1045 (2001).

[48] B. A. Inglis, E. L. Bossart, D. L. Buckley, E. D. W. III, and T. H. Mareci, "Visualization of Neural Tissue Water Compartments using Biexponential Diffusion Tensor MRI," Magn. Reson. Med. 45, 580-587 (2001).

[49] C. A. Clark, M. Hedehus, and M. E. Moseley, "Diffusion Time Dependence of the Apparent Diffusion Tensor in Healthy Human Brain and White Matter Disease," Magn. Reson. Med. 45, 1126-1129 (2001).

[50] C. A. Clark, M. Hedehus, and M. Moseley, "In Vivo Mapping of the Fast and Slow Diffusion Tensors in Human Brain," Magn. Reson. Med. 45, 623-628 (2002).

[51] L. R. Frank, "Anisotropy in High Angular Resolution Diffusion-Weighted MRI," Magn. Reson. Med. 45, 935-939 (2001). 
[52] D. S. Tuch, T. G. Reese, M. R. Wiegell, N. Makris, J. W. Belliveau, and V. J. Wedeen, "High Angular Resolution Diffusion Imaging Reveals Intravoxel White Matter Fiber Heterogeneity," Magn. Reson. Med. 48, 577-582 (2002).

[53] Y. Kadah, S. Ma, S. LaConte, I. Yassine, and X. Hu, "Robust Multi-component Modeling of Diffusion Tensor Magnetic Resonance Imaging Data," Proc. SPIE Medical Imaging (2005).

[54] V. Arsigny, P. Fillard, X. Pennec, and N. Ayache, "Fast and Simple Calculus on Tensors in the Log-Euclidean Framework," Procc. MICCAI: New York: Springer 6, 259-267 (2005), lecture Notes in Computer Science.

[55] X. Pennec, P. Fillard, and N. N. Ayache, "A Riemannian Framework for Tensor Computing," Inter. Jour. Comp. Vision 66, 41-66 (2006).

[56] P. Fletcher and S. Joshi, "Riemannian Geometry for the Statistical Analysis of Diffusion Tensor Data," Sig. Process. 87, 250-262 (2007).

[57] p. Batchelor, M. Moakher, D. Atkinson, F. Calamante, and A. Connelly, "A Rigorous Framework for Diffusion Tensor Calculus," Magn. Reson. Med. 53, 221-225 (2005).

[58] B. A. Barmpoutis, B. Jian, and T. M. Shepherd, "Symmetric Positive 4th Order Tensors and their Estimation from Diffusion Weighted MRI," Proc. of IPMI07: Info. Proc. Med Imag. pp. 308-319 (2007).

[59] C. deBoor, "On Calculating with B-Splines," J. Approx. Theory 6, 50-62 (1972). 
[60] G. Kindlmann, S. Jos, M. Niethammer, S. Haker, and C. Westin, "GeodesicLoxodromes for Diffusion Tensor Interpolation and Difference Measurement Export," Proc. of MICCAI pp. 1-9 (2007).

[61] E. Catmull and J. Clark, "Recursively Generated B-Spline Surfaces on Arbitrary Topological Surfaces," Computer-Aided Design 10, 350-355 (November 1978).

[62] D. Doo and M. Sabin, "Behavior of Recursive Subdivision Surfaces near Extraordinary Points," Comp. Aided Design 10, 356-360 (1978).

[63] H. Weimer and J. Warren, "Subdivision Schemes for Fluid Flow," Proc. 26th Ann. Conf. Comp. Graph. inter. Tech. pp. 111-120 (1999).

[64] T. M. Korn and G. Arthur, in Mathematical Handbook for Scientists and Engineers: Definitions, Theorems, and Formulas for Reference and Review (Dover Publications, 1999), p. 157160.

[65] G. K. Batchelor, in Introduction to Fluid Dynamics (Cambridge University Press, 2000), pp. 266-273.

[66] R. C. Beach, An Introduction to Curves and Surfaces of Computer-Aided Design (Van Nostrand Reinhold, 1991).

[67] G. Farin, Curves and Surfaces for Computer Aided Geometric Design: a Practical Guide (Academic Press Professional Inc. San Diego, CA, USA, 1993).

[68] I. Yassine and T. McGraw, "A Subdivision Approach to Tensor Field Interpolation," Workshop On Computational Diffusion MRI, MICCAI pp. 117124 (2008). 
[69] C. Liu, R. Bamme, B. Acar, and M. Moseley, "Characterizing Non-Gaussian Diffusion by using Generalized Diffusion Tensors," Magn. Reson. Med. 51, 924-937 (2004).

[70] F. Crick and E. Jones, "Ackwardness of Human Neuroanatomy," B. Nature 361, 109-110 (1993).

[71] L. Heimer and M. Robards, "Neuroanatomical Tract-Tracing Methods," Plenum, New York (1981).

[72] L. Heimer and L. Zaborszky, "Neuroanatomical Tract-Tracing Methods," Plenum, New York (1989).

[73] M. Mesulam, "Tracing Neural Connections with Horseradish Peroxidase," Wiley, Great Britain (1982).

[74] T. Conturo, N. Lori, T. Cul, E. Akbudak, A. Snyder, J. Shimony, R. McKinstry, H. Burton, and M. Raichle, "Tracking Neuronal Fiber Pathways in the Living Human Brain," Proc. Nat. Aca. Sc. USA 96, 10422-10427 (1999).

[75] C. Westin, S. Maier, B. Khidir, P. Everett, F. Jolesz, and R. Kikinis, "Image Processing for Diffusion Tensor Magnetic Resonance Imaging," MICCAI pp. 441-452 (1999).

[76] J. Maldjian and R. Grossman, "Future Applications of DWI in MS," J. Neurol. Sci. 186, S55S57 (2001).

[77] M. Filippi, M. Cercignani, M. Inglese, M. Horsfield, and G. Comi, "Diffusion Tensor Magnetic Resonance Imaging in Multiple Sclerosis," Neurology 56, 304-311 (2001). 
[78] T. Klingberg, M. Hedehus, E. Temple, T. Salz, J. Gabrieli, M. Moseley, and R. Poldrack, "Microstructure of Temporo-Parietal White Matter as a Basis for Readi," Neuron 25, 493-500 (2000).

[79] S. Rose, F. Chen, J. Chalk, F. Zelaya, W. Strugnell, M. B. ans J.Semple, and D. Doddrell, "Loss of Connectivity in Alzheimers Disease: an Evaluation of White Matter Tract Integrity with Colour Coded MR Diffusion Tensor Imaging," J. Neurol. Neurosurg. Psychiatry 69, 528-530 (2000).

[80] M. Bozzali, A. Falini, M. Franceschi, M. Cercignani, M. Zuffi, C. Scotti, G. Comi, and M. Filippi, "White Matter Damage in Alzheimers Disease Assessed in Vivo using Diffusion Tensor Magnetic Resonance," J. Neurol. Neurosurg. Psychiatry 72, 742-746 (2002).

[81] K. Lim, M. Hedehus, M. Moseley, A. de Crespigny, E. Sullivan, and A. Pfefferbaum, "Compromised White Matter Tract Integrity in Schizophrenia Inferred from Diffusion Tensor Imaging," Arch. Gen. Psychiatry 56, 367-374 (1999).

[82] J. Foong, M. Maier, C. Clark, G. Barker, D. Miller, and M. Ron, "Neuropathological Abnormalities of the Corpus Callosum in Schizophrenia: a Diffusion Tensor Imaging Study," J. Neurol. Neurosurg. Psychiatry 68, 242$244(2000)$.

[83] A. Field, A. Alexander, K. Hasan, K. Arfanakis, B. Witwer, R. Moftakhar, P. D. V. Haughton, and H. R. et al., "Diffusion-Tensor MR Imaging Patterns in White matter Tracts Altered by Neoplasm," Workshop on Diffusion MRI: Biophysical Issues. ISMRM pp. 137-140 (2002). 
[84] S. Mori, K. Frederiksen, P. van Zijl, B. Stieltjes, M. Kraut, S. M., and M. Pomper, "Brain White Matter Anatomy of Tumor Patients Evaluated with Diffusion Tensor Imaging," Ann. Neurol. 3, 377p-380 (51).

[85] A. Hoon, W. Lawrie, E. Melhem, E. Reinhardt, P. V. Zijl, M. Solaiyappan, H. Jiang, M. Johnston, and S. Mori, "Diffusion Tensor Imaging of Periventricular Leukomalacia Shows Affected Sensory Cortex White Matter Pathways," Neurology $\mathbf{5 9 ,} 752-756(2002)$.

[86] H. Mamata, C. Westin, K. Zou, and U. Girolami, "Combined Mapping of Functional Domains and Axonal Connectivity in Cat Visual Cortex using FMRI and DTI," ISMRM pp. 121-124 (2002).

[87] S. Mori, B. Crain, V. Chacko, and P. van Zijl, "Three-Dimensional Tracking of Axonal Projections in the Brain by Magnetic Resonance Imaging," Ann. Neurol. 45, 265-269 (1999).

[88] D. Jones, A. Simmons, S. Williams, and M. Horsfield, "Non-Invasive Assessment of Axonal Fiber Connectivity in the Human Brain via Diffusion Tensor MRI," Magn. Reson. Med. 42, 37-41 (1999).

[89] P. Basser, S. Pajevic, C. Pierpaoli, J. Duda, and A. Aldroubi, "In Vivo Fiber Tractography using DT-MRI Data," Magn. Reson. Med. 4, 625-632 (44).

[90] C. Tench, P. Morgan, M. Wilson, and L. Blumhardt, "White Matter Mapping using Diffusion Tensor MRI," Magn. Reson. Med. 47, 967-972 (2002).

[91] C. Pierpaoli, A. Barnett, S. Pajevic, R. Chen, L. Penix, A. Virta, and P. Basser, "Regularization of Diffusion-Based Direction Maps for the Tracking of Brain White Matter Fascicles," Neuroimage 12 (2000). 
[92] C. Pierpaoli, A. Barnett, S. Pajevic, R. Chen, L. Penix, A. Virta, and P. Basser, "Water Diffusion Changes in Wallerian Degeneration and their Dependence on White Matter Architecture," Neuroimage 13, 1174-1185 (2001).

[93] M. R. Wiegell, H. B. Larsson, and V. J. Wedeen, "Fiber Crossing in Human Brain Depicted with Diffusion Tensor MR Imaging," Radiology 217 (2000).

[94] M. Lazar, D. Weinstein, K. Hasan, and A. Alexander, "Bootstrap Analysis of DT-MRI Tractography Techniques: Streamlines and Tensorlines," Proc. Intl. Soc. Mag. Reson. Med. 9, 1527 (2001).

[95] M. Lazar, D. Weinstein, K. Hasan, and A. Alexander, "Axon Tractography with Tensorlines," Proc. Intl. Soc. Mag. Reson. Med 8, 483 (2000).

[96] M. Lazar et al., "White Matter Tractography using Diffusion Tensor Deflection," Human Brain Mapping pp. 306-321 (18).

[97] M. Lazar and A. Alexander, "White Matter Tractography Error Analysis in a Brain Diffusion Tensor Field," Proc. Intl. Soc. Mag. Reson. Med. 10 (2002).

[98] M. Lazar and A. Alexander, "Error Analysis of White Matter Tracking Algorithms (Streamlines and Tensorlines) for DT-MR," Proc. Intl. Soc. Mag. Reson. Med. 9, 506 (2001).

[99] J. Sethian, "Level Set Methods and Fast Marching Methods," Cambridge University Press (1999).

[100] G. Parker, "Tracing Fibre Tracts using Fast Marching," Proc. Intl. Soc. Mag. Reson. Med. 8, 85 (2000).

[101] O. Friman, G. Farneback, and C. Westin, "A Bayesian Approcah for Stochastic White Matter Tractography," Trans. Med. Imag. 25, 965-978 (2006). 
[102] R. Xue, P. van Zijl, B. Crain, M. Solaiyappan, and S. Mori, "In Vivo ThreeDimensional Reconstruction of Rat Brain Axonal Projections by Diffusion Tensor Imaging," Mag. Reson. Med. 43, 1123-1127 (1999).

[103] B. Stieltjes, W. Kaufmann, P. van Zij, K. Fredericksen, G. Pearlson, M. Solaiyappan, and S. Mori, "Diffusion Tensor Imaging and Axonal Tracking in the Human Brainstem," NeuroImage 14, 723-735 (2001).

[104] S. Mori et al., "Imaging Cortical Association Tracts in the Human Brain using Diffusion-Tensor Based Axonal Tracking," Mag. Reson. Med. 47, 215-223 (2002).

[105] H. Jianga, P. van Zijl, J. Kimc, G. Pearlsonc, and S. Mori, "DtiStudio: Resource Program for Diffusion Tensor Computation and Fiber Bundle Tracking," Comp. Meth. Prog. Biomed. 81, 106-116 (2006).

[106] K. Polthier and E. Preuss, "Identifying Vector Fields Singularities using a Discrete Hodge Decomposition," Visual. Math. 3, 113-134 (2003).

[107] H. Li, W. Chen, and I. Shen, "Segmentation of Discrete Vector Fields," IEEE Trans. Visual. Comp. Graph. 8, 289-300 (2006).

[108] Y. Tong, S. Lombeyda, A. Hirani, and M. Desbrun, "Discrete Multiscale Vector Field Decomposition," ACM Trans. Graph. 22, 445-452 (2003).

[109] T. Delmarcelle and L. Hesselink, "The Topology of Symmetric, Second-Order Tensor Fields," Proc. Conf. Visual. (1994).

[110] L. Hesselink, Y. Levy, and Y. Lavin, "The Topology of Symmetric, SecondOrder 3D Tensor Fields," IEEE Trans. Visual. Comp. Graph. pp. 1-11 (1997). 
[111] X. Zheng, B. Parlett, and A. Pang, "Topological Lines in 3D Tensor Fields and Discriminant Hessian Factorization," IEEE Trans. Visual. Comp. Graph. pp. 395-407 (2005).

[112] X. Tricoche, G. Kindlmann, and C. Westin, "Invariant Crease Lines for Topological and Structural Analysis of Tensor Fields," IEEE Trans. Visual. Comp. Graph. pp. 1627-1634 (2008).

[113] G. Arfken and H. Weber, "Mathematical Methods for Physics," Academic Press (2005).

[114] M. Descoteaux, R. Deriche, and C. Lenglet, "Diffusion Tensor Sharpening Improves White Matter Tractography," SPIE Medical Imaging (2007).

[115] M. Descoteaux, R. Deriche, T. Knosche, and A. Anwander, "Deterministic and Probabilistic Tractography Based on Complex Fiber Orientation Distributions," IEEE Trans. Med. Imag. 28, 269-286 (2009).

[116] M. W. T. Behrens, M. Jenkinsen-Berg, R. Nunes, S. clare, P. Mathews, J. Brady, and S. Smith, "Characterization and Propagation of Uncertainity in Diffusion-Weigthed MR Imaging," Mag. Reson. Med. 50, 1077-1088 (2003).

[117] D. Jones and C. Pierpaoli, "Confidence in Diffusion Tensor Magnetic Resonance Imaging using a Bootstrap Approach," Mag. Reson. Med. 5, 1143$1149(2005)$.

[118] B. Jian and B. Vemuri, "A Unified Computational Framework for Deconvolution to Reconstruct Multiple Fibers From Diffusion Weighted MRI," IEEE Trans. Med. Imag. 11, 1464-1471 (2006). 
[119] K. Jansons and A. D, "Persistent Angular Structure: New Insights from Diffusion Magnetic Resonance Imaging Data," Inv. Prob. 19, 1031-1046 (2003).

[120] E. Ozarslan, T. Shepher, B. Vemuri, S. Blackband, and T. Mareci, "Resolution of Complex Tissue Microarchitecture using the Diffusion Orientation Transform (DOT)," Neuroimage 31, 1086-1103 (2006).

[121] A. Leow, S. Zhu, L. Zhan, K. McMahon, G. de Zubicaray, M. Meredith, M. Wright, A. Toga, and P. Thompson, "The Tensor Distribution Function," Mag. Resosn. Med. 61, 205-214 (2009).

[122] D. S. Tuch, "Q-Ball Imaging," Mag. Reson. Imag. 53, 1358-1372 (2004).

[123] M. Descoteaux, E. Angelino, S. Fitzgibbons, and R. Deriche, "Regularized, Fast, and Robust Analytical Q-Ball Imaging," Mag. Reson. Med. 58, 497-510 (2007).

[124] Y. Chen, W. Guo, Q. Zeng, X. Yan, F. Huang, H. Zhang, G. He, B. C. Vemuri, , and Y. Liu, "Estimation, Smoothing, and Characterization of Apparent Diffusion Coefficient Profiles from High Angular Resolution DWI," IEEE Conf. Comp. Vis. Patt. Recog. pp. 588-593 (2004).

[125] Y. Chen, W. Guo1, Q. Zeng, X. Yan, M. Rao, and Y. Liu, "Apparent Diffusion Coefficient Approximation and Diffusion Anisotropy Characterization in DWI," PMI 2005 p. 246257 (2005).

[126] E. Scerri, "The Electronic Configration Model, Quantum Mechanics and Reduction," Brit. jour. Phil. Sci. 42, 309-325 (1991). 
[127] R. Geroch, in General Relativity from A to B (the University of Chicago Press, 1981), Chap. 5, pp. 181-186.

[128] P. Vancek and Z. Martinec, "Compilation of a Precise Regional Geoid," Manuscripta Geodaetica 19, 119-128 (1994).

[129] P. Zarka, R. Treumann, B. Ryabov, and V. Ryabov, "Magnetically-Driven Planetary Radio Emissions and Application to Extrasolar Planets," Astrophys. Space Sci. pp. 277-293 (2001).

[130] S. Hanany et al., "MAXIMA-1:A Measurement of the Cosmic Microwave Background Anisotropy on Angular Scales of 10'-5," Astrophysic. Jour. 545 (2000).

[131] M. Abramowitz and A. Stegun, in Handbook of Mathematical Functions with Formulas, Graphs, and Mathematical Tables (New York: Dover, 1965), Chap. 8, p. 3202 .

[132] H. S. Kim, "Relationship between Fiber Orientation Distribution Function and Mechanical Anisotropy of Thermally Point-Bonded Nonwovens," Fibers and Polymers 5, 177-181 (2004).

[133] P. Basser and S. Pajevic, "Statistical Artifacts in DT-MRI Data Caused by Background Noise," Magnetic Resonance in Medicine 44, 41-50 (2000).

[134] H. Gudbjartsson and S. Patz, "The Rician Distribution of Noisy MRI Data," Magnetic Resonance in Medecine 34, 910-914 (1995).

[135] L. Chang, C. Koay, C. Pierpaoli, and P. Basser, "Variance of Estimated DTIDerived Parameters via First Order Perturbation Methods," Magnetic Resonance in Medecine 57, 141-149 (2007). 
[136] G. Golub and C. V. Loan, in Matrix Computation (the Johns hopkins university Press, 1996), Chap. 7, pp. 320-340.

[137] K. Hahn, S. Prigarin, S. Heim, and K. Hasan, "Random Noise in Diffsion Tensor Imaging, its Destructive Impact and Some Corrections," Mathematics and visulaization II, 107-119 (2006).

[138] A. Anderson, "Theoretical Analysis of the Effects of Noise on Diffsion Tensor Imaging," Magnetic Resonance in Medecine 46, 1174-1188 (2001). 